\title{
Tunable Metal-Catalyzed Heterocyclization Reactions of Allenic Amino Alcohols: An Experimental and Theoretical Study
}

\author{
Benito Alcaide, ${ }^{* \dagger}$ Pedro Almendros, ${ }^{*}{ }^{, \dagger}$ Cristina Aragoncillo, ${ }^{\dagger}$ Gonzalo Gómez- \\ Campillos, ${ }^{\dagger}$ M. Teresa Quirós, ${ }^{\dagger}$ and Elena Soriano ${ }^{\ddagger}$ \\ ${ }^{\dagger}$ Grupo de Lactamas y Heterociclos Bioactivos, Departamento de Química Orgánica I, Unidad \\ Asociada al CSIC, Facultad de Química, Universidad Complutense de Madrid, 28040-Madrid, \\ Spain \\ ${ }^{\ddagger}$ Instituto de Química Orgánica General, IQOG-CSIC, Juan de la Cierva 3, 28006-Madrid, Spain \\ E-mail: alcaideb@quim.ucm.es; Palmendros@iqog.csic.es
}

Table of Contents

Additional computational results S1-S4

Cartesian coordinates

S5-S39

${ }^{1} \mathrm{H}$ NMR and ${ }^{13} \mathrm{C}$ NMR Spectra

S40-S62

\section{Additional computational results:}

\section{Formation of the Pd-catalyzed protonolysis products}

In presence of an unsaturated reactant, the cross-coupling from the $\sigma$-alkenyl intermediate $12_{6 \mathrm{Br}}$ is favored over a competitive proton shift process to yield protonolysis product 
$\left(\mathrm{PH}_{6 \mathrm{Br}}\right)$. Thus, the protonolysis step from $\mathbf{1}_{6 \mathrm{Br}}$ involves a less favored process both from kinetic and thermodynamic viewpoints, since our calculations reveal a higher activation barrier (transition structure $11.9 \mathrm{kcal} \mathrm{mol}^{-1}$ above $\mathbf{1 b}-\mathbf{P d}_{\mathrm{D}}$, and $4.7 \mathrm{kcal} \mathrm{mol}^{-1}$ higher than TS4 $\left._{6 \mathrm{Br}}\right)$ and less exergonicity $(-14.4 \mathrm{kcal} / \mathrm{mol})$ than the coupling-process, in agreement with the experimental absence of the protonolysis product.

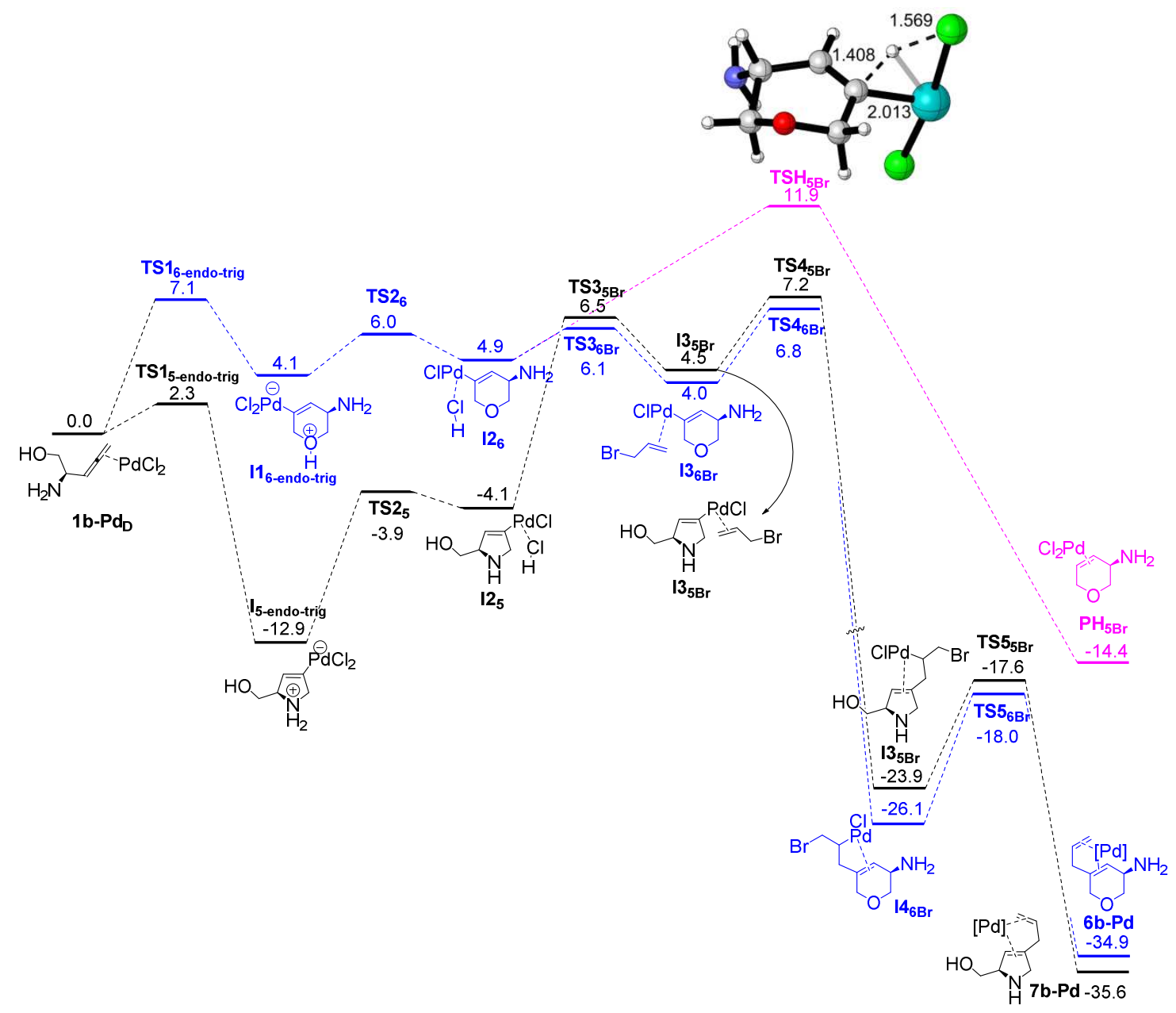

$\mathrm{C}\left(\mathrm{sp}^{2}\right)-\mathrm{H} . . . \pi$ interaction in the cross-coupling process with $\alpha$-allenic acetate

TS4 $_{6 \text { Al }}$ shows a $\mathrm{C}\left(\mathrm{sp}^{2}\right)-\mathrm{H} \ldots \pi$ interaction with the aromatic core $(2.504 \AA)$. Other conformation for $\mathbf{T S}_{6 \mathrm{Gl}}$ lacking this interaction, $\mathbf{T S} \mathbf{4}_{6 \mathrm{Al}}{ }_{\mathrm{Al}}$, is $2.8 \mathrm{kcal} \mathrm{mol}^{-1}$ higher in energy. 


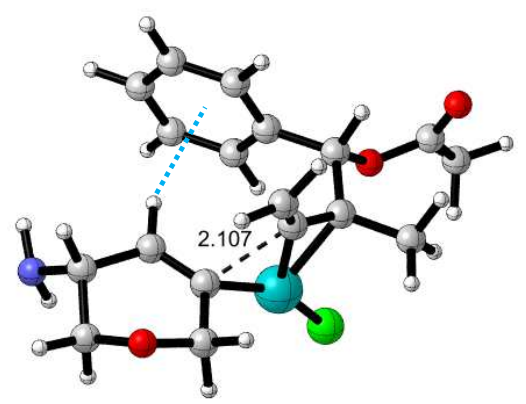

$\mathrm{TS}_{6 \mathrm{AI}}$

3.4

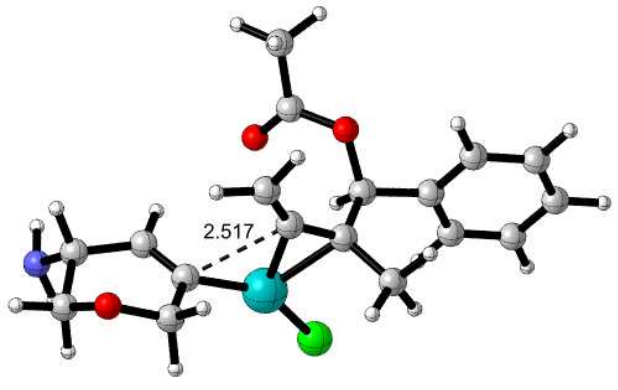

TS4' ${ }_{6 \mathrm{~A}}$

16.2

Analogously, this effect is also observed in the subsequent intermediate $\mathbf{1 4 _ { 6 \mathrm { Al } }}$ Vs $\mathbf{1 \mathbf { 4 } ^ { \prime }}{ }_{\mathrm{GA}} \mathrm{Al}$.

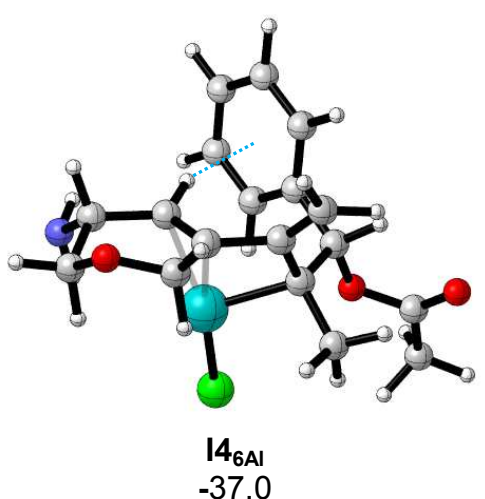

$-37.0$

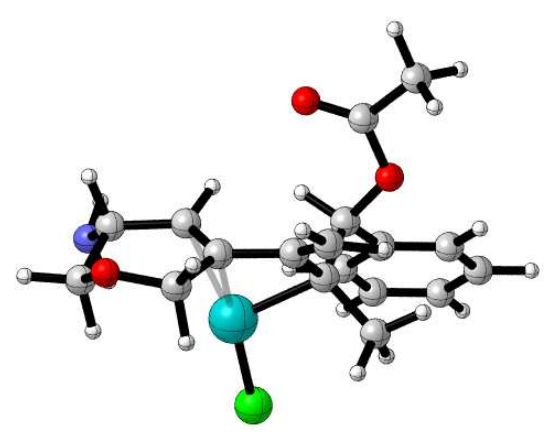

$14{ }^{\prime}{ }_{6 \mathrm{~A}}$ $-34.3$

The cross-coupling of the dihydropyrrole structure TS4 $_{5 \mathrm{Al}}$ shows a C $\left(\mathrm{sp}^{3}\right)-\mathrm{H} \ldots \pi(2.798$, $3.211 \AA$ ) , weaker than the $\mathrm{C}\left(\mathrm{sp}^{2}\right)-\mathrm{H} \ldots \pi$ in $\mathbf{T S} \mathbf{4}_{6 \mathrm{Al}}$, as expected. ${ }^{1}$ Other conformation lacking this interaction, $\mathbf{T S}^{\prime}{ }^{\prime}{ }_{\mathbf{A l}}$, is also less stable. On other hand, the transition structure involving a $\mathrm{C}\left(\mathrm{sp}^{2}\right)-\mathrm{H} \ldots \pi$ interaction would involve the coupling over the most hindered face, so it should be even less stable.
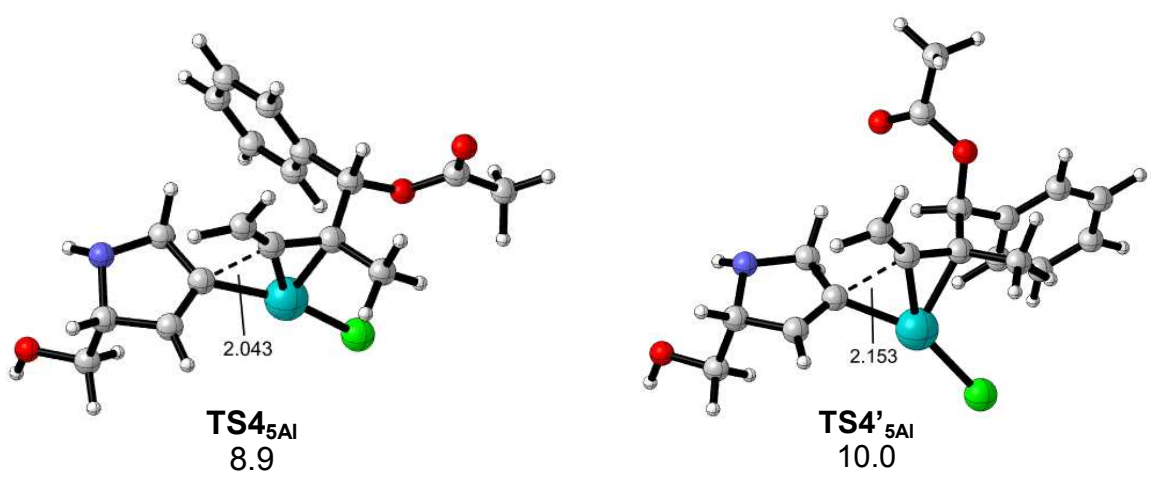

${ }^{1}$ S. Scheiner, Hydrogen Bonding: A Theoretical Perspective, Oxford University Press, 1997 
NBO analysis of the coupling processes

\begin{tabular}{|c|c|c|c|c|}
\hline Coupling reagent & Parameter & & $13_{5}$ & $13_{6}$ \\
\hline \multirow[t]{6}{*}{ Allyl bromide } & NPA charge & heterocycle & -0.07 & -0.06 \\
\hline & & alkene & +0.19 & +0.17 \\
\hline & & $\mathrm{PdCl}$ & -0.12 & -0.11 \\
\hline & Wiberg bond order & $\mathrm{Pd}-\mathrm{C} 1$ & 0.264 & 0.246 \\
\hline & & $\mathrm{Pd}-\mathrm{C} 2$ & 0.244 & 0.218 \\
\hline & & $\mathrm{Pd}-\mathrm{C}_{\mathrm{C}}$ & 0.694 & 0.700 \\
\hline \multirow[t]{6}{*}{$\alpha$-Allenic acetate } & NPA charge & heterocycle & -0.04 & -0.02 \\
\hline & & allene & +0.03 & +0.13 \\
\hline & & $\mathrm{PdCl}$ & +0.01 & -0.10 \\
\hline & Wiberg bond order & $\mathrm{Pd}-\mathrm{C} 1$ & 0.186 & 0.222 \\
\hline & & $\mathrm{Pd}-\mathrm{C} 2$ & 0.155 & 0.198 \\
\hline & & $\mathrm{Pd}-\mathrm{C}_{\mathrm{C}}$ & 0.727 & 0.696 \\
\hline
\end{tabular}




\section{$\underline{\text { Cartesian coordinates }}$}

\begin{tabular}{lccc}
\multicolumn{4}{l}{$\mathbf{1 b}-\mathrm{Au}_{\mathrm{D}}$} \\
6 & 4.245138000 & -0.033906000 & -0.452874000 \\
1 & 5.327718000 & -0.117207000 & -0.300236000 \\
6 & 3.562763000 & -0.096511000 & 0.901559000 \\
6 & 2.057813000 & -0.123856000 & 0.805815000 \\
6 & 1.392562000 & 0.030514000 & -0.289074000 \\
6 & 0.814683000 & 0.240922000 & -1.481460000 \\
1 & 0.689238000 & -0.582627000 & -2.173777000 \\
1 & 0.722347000 & 1.254374000 & -1.853658000 \\
17 & -0.854753000 & 2.290510000 & 0.344122000 \\
17 & -0.997669000 & -2.241982000 & -0.605467000 \\
8 & 3.910037000 & 1.111316000 & -1.188654000 \\
1 & 4.262563000 & 1.888736000 & -0.754371000 \\
1 & 1.514947000 & -0.265705000 & 1.734835000 \\
7 & 4.005609000 & -1.295557000 & 1.581213000 \\
1 & 5.003501000 & -1.280780000 & 1.738485000 \\
1 & 3.549648000 & -1.423285000 & 2.472972000 \\
1 & 3.803052000 & 0.834183000 & 1.445643000 \\
1 & 3.938625000 & -0.899242000 & -1.042497000 \\
79 & -0.977010000 & 0.022350000 & -0.121437000 \\
17 & -3.052589000 & -0.113155000 & 0.841526000
\end{tabular}

\begin{tabular}{lccc}
\multicolumn{4}{l}{ 1b-Au } \\
6 & 2.419401000 & 0.412347000 & -0.667817000 \\
6 & 1.302488000 & 1.075003000 & 0.115066000 \\
6 & 0.929344000 & 0.774628000 & 1.368556000 \\
6 & 0.780314000 & 0.577071000 & 2.638370000 \\
1 & -0.012684000 & -0.035814000 & 3.042857000 \\
1 & 1.467128000 & 1.045820000 & 3.332219000 \\
17 & -1.695997000 & 2.073716000 & -0.163125000 \\
17 & 0.178828000 & -2.149915000 & 0.225715000 \\
1 & 1.982824000 & -0.277906000 & -1.402100000 \\
1 & 0.936040000 & 2.010547000 & -0.298999000 \\
7 & 3.180956000 & 1.494943000 & -1.263818000 \\
1 & 4.072497000 & 1.136657000 & -1.579666000 \\
1 & 2.704762000 & 1.891407000 & -2.061679000 \\
6 & 3.333788000 & -0.395171000 & 0.225446000 \\
1 & 3.740089000 & 0.266874000 & 1.002072000 \\
1 & 2.761444000 & -1.193654000 & 0.702432000 \\
8 & 4.354284000 & -0.902359000 & -0.598361000 \\
1 & 4.910922000 & -1.493387000 & -0.094199000 \\
79 & -0.760993000 & -0.041468000 & -0.001515000 \\
17 & -2.726216000 & -0.981660000 & -0.707731000 \\
& & & \\
TS1 & 4 -exo-trig & & \\
6 & -3.203087000 & 1.168969000 & -0.776659000 \\
1 & -3.902383000 & 1.705579000 & -1.413386000 \\
6 & -3.449536000 & -0.314508000 & -0.586190000 \\
6 & -1.958923000 & -0.696642000 & -0.580114000 \\
6 & -1.128867000 & -0.649578000 & 0.557906000 \\
6 & -1.431107000 & -1.033768000 & 1.794580000 \\
1 & -0.704926000 & -0.962590000 & 2.593156000 \\
1 & -2.377440000 & -1.508251000 & 2.021250000
\end{tabular}




$\begin{array}{lrrr}17 & 0.119222000 & 2.155320000 & 0.597706000 \\ 17 & 1.221911000 & -2.227966000 & -0.499820000 \\ 8 & -1.914185000 & 1.068916000 & -1.402825000 \\ 1 & -1.256406000 & 1.631521000 & -0.928748000 \\ 1 & -1.561905000 & -1.233068000 & -1.436883000 \\ 7 & -4.194690000 & -0.749143000 & 0.551780000 \\ 1 & -4.200740000 & -0.089001000 & 1.313704000 \\ 1 & -5.131962000 & -1.039655000 & 0.333897000 \\ 1 & -3.890393000 & -0.746889000 & -1.483886000 \\ 1 & -3.114623000 & 1.672431000 & 0.189513000 \\ 79 & 0.809535000 & -0.039722000 & 0.099171000 \\ 17 & 3.005512000 & 0.634633000 & -0.340244000\end{array}$

TS1 5-exo-dig $_{\text {-ex }}$

$\begin{array}{lccc}6 & 3.617986000 & 1.025807000 & 0.402408000 \\ 1 & 3.135856000 & 1.724654000 & 1.086896000 \\ 6 & 3.619220000 & -0.378701000 & 0.998314000 \\ 6 & 2.221391000 & -0.876030000 & 0.769468000 \\ 6 & 1.634286000 & -0.606795000 & -0.353238000 \\ 6 & 0.703457000 & -0.641233000 & -1.372579000 \\ 1 & 0.545043000 & -1.612984000 & -1.828160000 \\ 1 & 0.715934000 & 0.185622000 & -2.072298000 \\ 17 & -0.031403000 & 2.157722000 & -0.252341000 \\ 17 & -1.668943000 & -2.199525000 & -0.072548000 \\ 8 & 2.911235000 & 0.965629000 & -0.822142000 \\ 1 & 2.178844000 & 1.609580000 & -0.794676000 \\ 1 & 1.655543000 & -1.394424000 & 1.534414000 \\ 7 & 4.571780000 & -1.211777000 & 0.284136000 \\ 1 & 5.520962000 & -0.987583000 & 0.550936000 \\ 1 & 4.421487000 & -2.192732000 & 0.470573000 \\ 1 & 3.806632000 & -0.312765000 & 2.076157000 \\ 1 & 4.639979000 & 1.351783000 & 0.205082000 \\ 79 & -0.965631000 & -0.007306000 & -0.151588000 \\ 17 & -2.794116000 & 0.737402000 & 1.070093000 \\ & & & \\ \text { TS1 } & \text { 5-endo-dig } & & \\ 6 & -2.624872000 & 0.285071000 & -1.388507000 \\ 1 & -1.862343000 & -0.170300000 & -2.025090000 \\ 6 & -2.012561000 & 1.430363000 & -0.601900000 \\ 6 & -1.246412000 & 0.831970000 & 0.687831000 \\ 6 & -1.942080000 & -0.086592000 & 1.417162000 \\ 6 & -2.435878000 & -0.916014000 & 2.264423000 \\ 1 & -1.815779000 & -1.283113000 & 3.074283000 \\ 1 & -3.453505000 & -1.271694000 & 2.182143000 \\ 17 & -0.397503000 & -2.108744000 & -0.504493000 \\ 17 & 1.556204000 & 1.946022000 & 0.685049000 \\ 8 & -3.188811000 & -0.656106000 & -0.517276000 \\ 1 & -2.598768000 & -1.427673000 & -0.511951000 \\ 1 & -0.995795000 & 1.698965000 & 1.303567000 \\ 1 & -2.812887000 & 2.018138000 & -0.145097000 \\ 1 & -3.405691000 & 0.698535000 & -2.028054000 \\ 7 & -1.257040000 & 2.272994000 & -1.434685000 \\ 1 & -0.938945000 & 3.122653000 & -0.994537000 \\ 1 & -0.470698000 & 1.813563000 & -1.873590000 \\ 79 & 0.617891000 & -0.085854000 & 0.096448000 \\ 17 & 2.689631000 & -0.916544000 & -0.574328000 \\ & & & \\ 17 & & \end{array}$




\begin{tabular}{|c|c|c|c|}
\hline \multicolumn{4}{|c|}{$\mathrm{TS}_{1}$ 6-endo-trig } \\
\hline \multicolumn{4}{|c|}{-0.046235000} \\
\hline 1 & -4.963251000 & 0.095435000 & 0.788201000 \\
\hline 6 & -3.498975000 & 0.129001000 & -0.795799000 \\
\hline 6 & -2.005159000 & 0.037591000 & -0.990930000 \\
\hline 6 & -1.164543000 & 0.191244000 & 0.006619000 \\
\hline 6 & -1.326635000 & 0.525775000 & 1.352612000 \\
\hline 1 & -1.013023000 & 1.514157000 & 1.674568000 \\
\hline 1 & -1.263790000 & -0.257647000 & 2.101868000 \\
\hline 17 & 0.576189000 & -2.267149000 & 0.456315000 \\
\hline 17 & 1.040430000 & 2.278530000 & -0.342887000 \\
\hline 8 & -3.193704000 & 0.841998000 & 1.558825000 \\
\hline 1 & -3.356201000 & 1.754562000 & 1.294495000 \\
\hline 1 & -1.657187000 & -0.199220000 & -1.989028000 \\
\hline 1 & -3.630791000 & -1.050223000 & 0.997335000 \\
\hline 1 & -3.852682000 & 1.109327000 & -1.137857000 \\
\hline 7 & -4.254249000 & -0.873150000 & -1.521538000 \\
\hline 1 & -4.286346000 & -0.674358000 & -2.510210000 \\
\hline 1 & -3.838529000 & -1.788874000 & -1.405661000 \\
\hline 79 & 0.916247000 & -0.018498000 & -0.011443000 \\
\hline 17 & 3.214530000 & -0.287264000 & -0.238750000 \\
\hline \multicolumn{4}{|c|}{ TS1 $1_{\text {3-exo-trig }}$} \\
\hline 6 & -4.185884000 & -0.651822000 & -0.443763000 \\
\hline 1 & -3.836825000 & -1.331098000 & -1.233662000 \\
\hline 6 & -3.007532000 & 0.122609000 & 0.087054000 \\
\hline 6 & -1.815206000 & -0.710060000 & 0.362334000 \\
\hline 6 & -0.823889000 & -0.345799000 & 1.255150000 \\
\hline 6 & -0.776097000 & -0.141591000 & 2.561389000 \\
\hline 1 & 0.094360000 & 0.289561000 & 3.035323000 \\
\hline 1 & -1.578380000 & -0.469203000 & 3.212968000 \\
\hline 17 & 0.500677000 & 2.260989000 & 0.356248000 \\
\hline 17 & 1.071706000 & -2.317449000 & -0.160785000 \\
\hline 8 & -5.105510000 & 0.282793000 & -0.932942000 \\
\hline 1 & -5.885640000 & -0.165418000 & -1.257610000 \\
\hline 1 & -1.635726000 & -1.543162000 & -0.312914000 \\
\hline 7 & -2.309784000 & 0.820047000 & -0.964460000 \\
\hline 1 & -2.733181000 & 0.838923000 & -1.876767000 \\
\hline 1 & -1.720114000 & 1.600618000 & -0.706677000 \\
\hline 1 & -3.301752000 & 0.769352000 & 0.914143000 \\
\hline 1 & -4.601789000 & -1.262894000 & 0.363681000 \\
\hline 79 & 0.896518000 & -0.018766000 & 0.068462000 \\
\hline 17 & 2.841637000 & 0.357233000 & -1.152241000 \\
\hline \multicolumn{4}{|c|}{ TS1 4-exo-dig } \\
\hline 6 & -4.129964000 & -0.604476000 & 0.866165000 \\
\hline 1 & -3.906671000 & -1.097679000 & 1.817745000 \\
\hline 6 & -3.475321000 & 0.775931000 & 0.857252000 \\
\hline 6 & -2.002966000 & 0.677840000 & 0.998898000 \\
\hline 6 & -1.507119000 & 0.989046000 & -0.169992000 \\
\hline 6 & -0.597985000 & 1.089813000 & -1.200880000 \\
\hline 1 & -0.218886000 & 2.077899000 & -1.437065000 \\
\hline 1 & -0.791888000 & 0.446770000 & -2.054755000 \\
\hline 17 & -0.248639000 & -1.977045000 & -0.550395000 \\
\hline 17 & 1000 & 2.014504000 & 0.2684 \\
\hline
\end{tabular}




\begin{tabular}{|c|c|c|c|}
\hline 6 & 3.298365000 & -1.211477000 & -0.672479000 \\
\hline 1 & 3.878451000 & -1.772319000 & -1.397931000 \\
\hline 6 & 3.563201000 & 0.281769000 & -0.486652000 \\
\hline 6 & 2.040175000 & 0.517562000 & -0.725747000 \\
\hline 6 & 1.092930000 & 0.791092000 & 0.367586000 \\
\hline 6 & 1.345452000 & 1.595648000 & 1.383904000 \\
\hline 1 & 0.569518000 & 1.866525000 & 2.086844000 \\
\hline 1 & 2.318780000 & 2.048269000 & 1.526145000 \\
\hline 17 & 0.060854000 & -2.130469000 & 0.571017000 \\
\hline 17 & -1.448610000 & 2.148912000 & -0.538272000 \\
\hline 8 & 1.954627000 & -0.945607000 & -1.201592000 \\
\hline 1 & 1.236967000 & -1.475171000 & -0.673722000 \\
\hline 1 & 1.796894000 & 1.109251000 & -1.607203000 \\
\hline 7 & 4.154787000 & 0.754449000 & 0.715147000 \\
\hline 1 & 3.836814000 & 0.273137000 & 1.543366000 \\
\hline 1 & 5.160385000 & 0.769604000 & 0.682364000 \\
\hline 1 & 4.118992000 & 0.688470000 & -1.329130000 \\
\hline 1 & 3.204409000 & -1.753472000 & 0.269284000 \\
\hline 79 & -0.815003000 & 0.031024000 & 0.081157000 \\
\hline 17 & -2.994435000 & -0.828386000 & -0.156532000 \\
\hline \multicolumn{4}{|c|}{$11_{5 \text {-exo-dig }}$} \\
\hline 6 & -3.109145000 & -1.055903000 & 0.696372000 \\
\hline 1 & -2.431669000 & -1.382217000 & 1.485980000 \\
\hline 6 & -3.666121000 & 0.340234000 & 0.939323000 \\
\hline 6 & -2.613896000 & 38000 & 0.292031000 \\
\hline 6 & -1.860468000 & 1000 & -0.533382000 \\
\hline 6 & 4000 & 7000 & -1.337 \\
\hline 1 & -0.530326000 & 1.772559000 & -1.518001000 \\
\hline 1 & -0.659134000 & 0.133019000 & -2.253519000 \\
\hline 17 & 0.160538000 & -2.201380000 & -0.443775000 \\
\hline 17 & 1.519250000 & 2.249742000 & 0.100001000 \\
\hline 8 & -2.292116000 & -0.896664000 & -0.512414000 \\
\hline 1 & -1.417347000 & -1.536200000 & -0.520928000 \\
\hline 1 & -2.499552000 & 000 & 0.444262000 \\
\hline 7 & -4.955138000 & 1000 & 0.286545000 \\
\hline 1 & -5.7 & 000 & 000 \\
\hline 1 & -5.052181000 & 1.440138000 & -0.104856000 \\
\hline 1 & -3.709188000 & 0.523504000 & 2.017137000 \\
\hline 1 & -3.866382000 & -1.800600000 & 0.476574000 \\
\hline 79 & 0.950776000 & 0.040773000 & -0.163368000 \\
\hline 17 & 2.757274000 & -0.718263000 & 1.150581000 \\
\hline \multicolumn{4}{|c|}{$\mid 1_{5 \text {-endo-dig }}$} \\
\hline 6 & -2.688974000 & 0.135028000 & -1.258047000 \\
\hline 1 & -2.120191000 & -0.349314000 & -2.049721000 \\
\hline 6 & -1.980902000 & 1.378384000 & -0.729474000 \\
\hline 6 & -1.289852000 & 0.868839000 & 0.543117000 \\
\hline 6 & -2.205943000 & -0.137987000 & 1.044551000 \\
\hline 6 & -2.612291000 & -0.557623000 & 2.223421000 \\
\hline 1 & -3.328125000 & -1.358362000 & 2.321791000 \\
\hline 1 & -2.211761000 & -0.101203000 & 3.114753000 \\
\hline 17 & -0.357701000 & -2.153248000 & -0.516993000 \\
\hline 17 & 1.494025000 & 2.008002000 & 0.618462000 \\
\hline 8 & -2.732490000 & -0.816097000 & -0.130395000 \\
\hline & -1.952295000 & 3095 & \\
\hline
\end{tabular}




$\begin{array}{lrrr}1 & -1.053932000 & 1.644581000 & 1.262240000 \\ 1 & -2.737969000 & 2.100562000 & -0.408095000 \\ 1 & -3.713657000 & 0.295833000 & -1.573441000 \\ 7 & -1.227849000 & 1.998017000 & -1.783945000 \\ 1 & -0.829777000 & 2.874602000 & -1.476761000 \\ 1 & -0.452838000 & 1.413371000 & -2.075590000 \\ 79 & 0.603545000 & -0.047807000 & 0.100718000 \\ 17 & 2.736800000 & -0.959553000 & -0.345866000\end{array}$

\begin{tabular}{lrrr}
\multicolumn{1}{l}{$\mathbf{I}_{\text {6-endo-trig }}$} \\
6 & -3.910270000 & 0.387355000 & 0.504627000 \\
1 & -4.918399000 & 0.787290000 & 0.456112000 \\
6 & -3.457442000 & -0.282485000 & -0.772242000 \\
6 & -1.960525000 & -0.420282000 & -0.805587000 \\
6 & -1.139597000 & 0.143365000 & 0.065889000 \\
6 & -1.585405000 & 1.039670000 & 1.131823000 \\
1 & -1.015438000 & 1.966094000 & 1.178509000 \\
1 & -1.667624000 & 0.581426000 & 2.116346000 \\
17 & 0.658728000 & -2.277294000 & 0.538047000 \\
17 & 0.934352000 & 2.247605000 & -0.505106000 \\
8 & -3.023983000 & 1.522040000 & 0.855513000 \\
1 & -2.968746000 & 2.140068000 & 0.109704000 \\
1 & -1.571741000 & -1.061267000 & -1.589005000 \\
1 & -3.820814000 & -0.284387000 & 1.355622000 \\
1 & -3.798016000 & 0.311067000 & -1.628050000 \\
7 & -4.186478000 & -1.537939000 & -0.807138000 \\
1 & -4.307753000 & -1.873597000 & -1.750407000 \\
1 & -3.687715000 & -2.250179000 & -0.288116000 \\
79 & 0.905180000 & -0.036665000 & 0.001336000 \\
17 & 3.234193000 & -0.207750000 & -0.151028000
\end{tabular}

$\begin{array}{lrrr}\mathbf{I}_{\text {3-exo-trig }} & & & \\ 6 & -4.464750000 & -0.513675000 & -0.506738000 \\ 1 & -4.210690000 & -1.024649000 & -1.445652000 \\ 6 & -3.209711000 & -0.114770000 & 0.194409000 \\ 6 & -1.911982000 & -0.724287000 & -0.139580000 \\ 6 & -0.799951000 & -0.776682000 & 0.824355000 \\ 6 & -0.945175000 & -1.276131000 & 2.034554000 \\ 1 & -0.117053000 & -1.360010000 & 2.724086000 \\ 1 & -1.905945000 & -1.643176000 & 2.385768000 \\ 17 & 0.093087000 & 2.128773000 & 0.643448000 \\ 17 & 1.494668000 & -2.160714000 & -0.530656000 \\ 8 & -5.202948000 & 0.654003000 & -0.732387000 \\ 1 & -6.069803000 & 0.436839000 & -1.075224000 \\ 1 & -1.890520000 & -1.452093000 & -0.944848000 \\ 7 & -2.252626000 & 0.641765000 & -0.638950000 \\ 1 & -2.479589000 & 0.759386000 & -1.617543000 \\ 1 & -1.668837000 & 1.392635000 & -0.209431000 \\ 1 & -3.313674000 & 0.318436000 & 1.180941000 \\ 1 & -4.995730000 & -1.233607000 & 0.123237000 \\ 79 & 0.949823000 & -0.004687000 & 0.078606000 \\ 17 & 2.941493000 & 0.907553000 & -0.785821000 \\ & & & \\ \mathbf{1} \mathbf{1}_{\text {4-exo-dig }} & -3.211818000 & -0.005525000 & 1.679417000 \\ \mathbf{1} & -3.686895000 & 0.874139000 & 2.125043000\end{array}$




$\begin{array}{lrrr}6 & -3.402248000 & 0.075420000 & 0.172985000 \\ 6 & -2.700811000 & 1.176461000 & -0.556892000 \\ 6 & -1.819550000 & 0.355496000 & -1.130977000 \\ 6 & -0.572217000 & 0.337345000 & -1.839119000 \\ 1 & -0.335287000 & 1.296038000 & -2.285098000 \\ 1 & -0.454365000 & -0.497611000 & -2.522776000 \\ 17 & 0.363732000 & -2.289831000 & -0.483168000 \\ 17 & 0.901678000 & 2.312249000 & -0.016684000 \\ 8 & -1.873113000 & -0.147560000 & 2.033287000 \\ 1 & -1.395657000 & 0.685818000 & 1.987581000 \\ 1 & -2.803589000 & 2.246828000 & -0.537625000 \\ 7 & -2.431498000 & -0.876527000 & -0.537217000 \\ 1 & -1.764022000 & -1.389505000 & 0.063053000 \\ 1 & -2.838568000 & -1.524338000 & -1.206059000 \\ 1 & -4.435979000 & -0.138410000 & -0.104340000 \\ 1 & -3.733064000 & -0.883926000 & 2.063916000 \\ 79 & 0.805740000 & 0.006185000 & -0.253639000 \\ 17 & 2.266308000 & -0.344573000 & 1.558411000\end{array}$

$\begin{array}{lccc}\text { I1 } & \text { 4-endo-dig } \\ 6 & 3.672563000 & -0.239668000 & -0.772802000 \\ 1 & 3.999935000 & -0.784039000 & 0.124200000 \\ 6 & 2.319401000 & 0.378701000 & -0.519271000 \\ 6 & 1.313996000 & -0.541705000 & 0.170126000 \\ 6 & 1.732726000 & 0.041052000 & 1.455281000 \\ 6 & 1.722741000 & -0.186975000 & 2.749669000 \\ 1 & 1.246546000 & -1.081145000 & 3.123786000 \\ 1 & 2.158704000 & 0.500503000 & 3.458730000 \\ 17 & -0.245826000 & 2.264376000 & -0.273429000 \\ 17 & -1.138853000 & -2.295740000 & 0.237745000 \\ 8 & 3.500162000 & -1.084149000 & -1.868379000 \\ 1 & 4.298699000 & -1.584448000 & -2.031872000 \\ 1 & 1.395911000 & -1.609029000 & 0.006704000 \\ 1 & 1.983495000 & 0.972243000 & -1.366015000 \\ 1 & 4.405667000 & 0.554515000 & -0.974503000 \\ 7 & 2.353449000 & 1.210632000 & 0.758629000 \\ 1 & 3.246944000 & 1.561453000 & 1.091590000 \\ 1 & 1.636465000 & 1.960097000 & 0.641665000 \\ 79 & -0.738817000 & -0.045662000 & -0.085295000 \\ 17 & -3.030016000 & 0.420038000 & -0.328870000 \\ & & & \\ \mathbf{I} \mathbf{1}_{\text {5-endo-trig }} & 3.987583000 & -0.840203000 & -0.399620000 \\ 6 & 3.329655000 & -1.498920000 & 0.179383000 \\ 6 & 3.258780000 & 0.449832000 & -0.703503000 \\ 6 & 1.847195000 & 0.229243000 & -1.147019000 \\ 6 & 0.994609000 & 0.360089000 & -0.143576000 \\ 6 & 1.676990000 & 0.784176000 & 1.103691000 \\ 1 & 1.774076000 & -0.034111000 & 1.819793000 \\ 1 & 1.215087000 & 1.639834000 & 1.592061000 \\ 17 & -1.297320000 & 2.268803000 & -0.108047000 \\ 17 & -0.364623000 & -2.273723000 & 0.170876000 \\ 8 & 5.151284000 & -0.492593000 & 0.318269000 \\ 1 & 5.637335000 & -1.279378000 & 0.564806000 \\ 1 & 1.617507000 & -0.109543000 & -2.145061000 \\ 1 & 4.211589000 & -1.339749000 & -1.345380000\end{array}$




$\begin{array}{llll}1 & 3.873179000 & 1.085656000 & -1.342137000 \\ 7 & 3.061014000 & 1.177268000 & 0.610133000 \\ 1 & 3.826654000 & 0.939129000 & 1.243123000 \\ 1 & 3.075409000 & 2.178687000 & 0.443966000 \\ 79 & -0.985272000 & -0.038605000 & -0.034536000 \\ 17 & -3.276430000 & -0.508652000 & 0.092515000\end{array}$

\section{TS2 60 -Au}

$6 \quad-3.537205000$

$1-3.528726000$

$6-3.354107000$

$6-1.880811000$

$6-1.143887000$

$6-1.823558000$

$-1.134680000$

$-2.513780000$

$\begin{array}{lr}17 & -2.513780000 \\ 17 & 1.142559000\end{array}$

$\begin{array}{ll}17 & 0.251111000\end{array}$

$8-2.528098000$

-2.528098000
-1.049258000

$-1.471373000$

$-4.504153000$

$-3.770198000$

$-4.112386000$

$-4.656253000$

$-3.499864000$

$\begin{array}{ll}79 & 0.867638000\end{array}$

$17 \quad 3.137726000$

0.983584000

$-0.227748000$

1.703500000

$-1.046001000$

$-0.444068000$

$-0.786254000$

$-0.758179000$

$-0.866002000$

$-0.390235000$

0.165715000

0.336845000

1.287273000

0.771467000

2.009055000

$-0.305933000$

1.841588000

$-2.353093000$

2.347340000

1.418517000

2.176094000

$-1.216335000$

1.027061000

$-0.423799000$

$-1.429474000$

$-2.027901000$

$-2.028180000$

$-0.103030000$

0.194856000

$-0.191582000$

0.686395000

0.055260000

$-1.756840000$

0.277436000

$-1.794157000$

$-0.011351000$

$-0.613188000$

0.524241000

0.016306000

0.520687000

$-0.217107000$

12 60 -Au

$\begin{array}{ll}6 & 2.957543000 \\ 1 & 4.025438000 \\ 6 & 2.700508000 \\ 6 & 1.260675000 \\ 6 & 0.572392000 \\ 6 & 1.051389000 \\ 1 & 0.765562000 \\ 1 & 0.620119000 \\ 17 & -2.091546000 \\ 17 & 2.981603000 \\ 8 & 2.457373000 \\ 1 & 2.961750000 \\ 1 & 0.879537000 \\ 1 & 2.489795000 \\ 1 & 3.350749000 \\ 7 & 3.029024000 \\ 1 & 3.063293000 \\ 1 & 2.385871000 \\ 79 & -1.404617000 \\ 17 & -0.989738000\end{array}$

$-1.593040000$

$-0.343925000$

$-1.706313000$

$-0.514486000$

$-1.013273000$

1.034368000

$-0.505473000$

1.107413000

$-0.425262000$

0.002077000

$-0.698577000$

$-1.382634000$

0.101481000

$-2.066073000$

$-1.636155000$

$-1.752462000$

$-1.865710000$

2.349485000

$-0.849064000$

$-0.752224000$

$-0.869115000$

1.090544000

$-1.361970000$

$-0.227170000$

$-1.180386000$

$-2.583812000$

2.079854000

$-0.147036000$

$-0.415144000$

$-2.006481000$

1.190505000

$-1.633379000$

2.020615000

$-2.786424000$

2.956939000

2.003026000

0.123974000

0.060162000

2.175709000

0.964833000

\section{TS3 60 -Au}

$6 \quad-3.803891000$

$1-4.861352000$

$-0.341101000$

0.600549000

$-0.220839000$

0.823059000

0.338463000

$-0.714132000$

$6-1.956633000$

0.451394000

$-0.815062000$ 


$\begin{array}{lrrr}6 & -1.146611000 & 0.210371000 & 0.228506000 \\ 6 & -1.707618000 & -0.099852000 & 1.580016000 \\ 1 & -1.195376000 & 0.478928000 & 2.349810000 \\ 1 & -1.526057000 & -1.162324000 & 1.792165000 \\ 17 & 0.655465000 & -2.295198000 & 0.036708000 \\ 17 & 0.667052000 & 2.378862000 & 0.000069000 \\ 8 & -3.072121000 & 0.213127000 & 1.660069000 \\ 1 & -0.672766000 & 1.571430000 & 0.051614000 \\ 1 & -1.548878000 & 0.699405000 & -1.791754000 \\ 1 & -3.596482000 & -1.417594000 & 0.507541000 \\ 1 & -3.891818000 & 1.340885000 & -0.728478000 \\ 7 & -4.019660000 & -0.419613000 & -1.806885000 \\ 1 & -3.979700000 & 0.065662000 & -2.690522000 \\ 1 & -3.576827000 & -1.323285000 & -1.908354000 \\ 79 & 0.917520000 & -0.022277000 & -0.004882000 \\ 17 & 3.236567000 & -0.106943000 & -0.268013000\end{array}$

\section{0-Au}

$\begin{array}{lrrr}6 & -3.274608000 & 0.422085000 & 0.240798000 \\ 1 & -4.257855000 & 0.593852000 & 0.672203000 \\ 6 & -2.420785000 & -0.374544000 & 1.219604000 \\ 6 & -1.341644000 & -1.144362000 & 0.510643000 \\ 6 & -1.250544000 & -1.180904000 & -0.855825000 \\ 6 & -2.274559000 & -0.465368000 & -1.679919000 \\ 1 & -2.518321000 & -1.080562000 & -2.545967000 \\ 1 & -1.875889000 & 0.486709000 & -2.053136000 \\ 17 & -0.272061000 & 2.094780000 & -0.384041000 \\ 17 & 1.685560000 & -2.072969000 & 0.179554000 \\ 8 & -3.463303000 & -0.280610000 & -0.964240000 \\ 1 & -0.608592000 & -1.911120000 & -1.334936000 \\ 1 & -0.774903000 & -1.856052000 & 1.101472000 \\ 1 & -2.809810000 & 1.394287000 & 0.054911000 \\ 1 & -3.052327000 & -1.156561000 & 1.659517000 \\ 7 & -1.988704000 & 0.491888000 & 2.290742000 \\ 1 & -1.537866000 & -0.003279000 & 3.045492000 \\ 1 & -1.369711000 & 1.218764000 & 1.951508000 \\ 79 & 0.675546000 & 0.001244000 & -0.071587000 \\ 17 & 2.729872000 & 1.006185000 & 0.096913000 \\ & & & \\ \text { TS2 } 4 \text { N-Au } & & & \\ 6 & -3.185740000 & 0.377449000 & -1.320017000 \\ 1 & -3.207447000 & 0.254852000 & -2.406565000 \\ 6 & -2.963024000 & -0.982855000 & -0.670669000 \\ 6 & -1.655592000 & -1.664862000 & -0.933334000 \\ 6 & -1.329881000 & -1.550616000 & 0.373495000 \\ 6 & -0.112775000 & -1.689436000 & 1.138166000 \\ 1 & 0.508285000 & -2.538309000 & 0.885453000 \\ 1 & -0.192533000 & -1.502170000 & 2.203880000 \\ 17 & -0.622116000 & 1.489285000 & 1.738688000 \\ 17 & 2.268458000 & -1.453618000 & -0.779022000 \\ 8 & -2.251070000 & 1.335450000 & -0.914052000 \\ 1 & -1.442411000 & 1.333898000 & -1.443294000 \\ 1 & -1.108293000 & -1.935638000 & -1.821476000 \\ 7 & -2.517875000 & -0.846785000 & 0.756194000 \\ 1 & -1.675879000 & 1.034067000 & 1.069506000 \\ 1 & -3.098328000 & -1.290515000 & 1.457324000\end{array}$




\begin{tabular}{lrrr}
1 & -3.854271000 & -1.602415000 & -0.791452000 \\
1 & -4.165293000 & 0.750279000 & -1.015289000 \\
79 & 0.788144000 & 0.014974000 & 0.216863000 \\
17 & 1.123570000 & 1.883090000 & -1.240671000 \\
\multicolumn{1}{l}{$\mathbf{I}_{\text {LN-Au }}$} & & & \\
6 & -2.287275000 & -1.698806000 & -0.677832000 \\
1 & -1.983636000 & -2.710813000 & -0.958385000 \\
6 & -1.991116000 & -1.515920000 & 0.797467000 \\
6 & -0.568219000 & -1.381802000 & 1.321518000 \\
6 & -0.946780000 & -0.067969000 & 1.769594000 \\
6 & -0.019436000 & 0.980697000 & 1.970132000 \\
1 & 0.781113000 & 0.819401000 & 2.681006000 \\
1 & -0.330531000 & 2.006949000 & 1.827960000 \\
17 & -2.630787000 & 2.166217000 & -1.021581000 \\
17 & 2.208661000 & 1.669857000 & -0.449966000 \\
8 & -1.709225000 & -0.745904000 & -1.522598000 \\
1 & -0.809799000 & -1.024969000 & -1.770354000 \\
1 & 0.037350000 & -2.136034000 & 1.801603000 \\
7 & -2.227408000 & -0.146386000 & 1.340052000 \\
1 & -2.153332000 & 1.013067000 & -1.417297000 \\
1 & -2.709862000 & 0.602862000 & 0.852688000 \\
1 & -2.567464000 & -2.261009000 & 1.351148000 \\
1 & -3.369775000 & -1.634830000 & -0.809204000 \\
79 & 0.787044000 & -0.048284000 & 0.234456000 \\
17 & 1.308717000 & -1.586984000 & -1.491159000
\end{tabular}

$\mathrm{TS}_{4 \mathrm{~N}-\mathrm{Au}}$

$\begin{array}{lrrr}6 & 3.746417000 & -0.559683000 & 1.347106000 \\ 1 & 4.483825000 & -1.359979000 & 1.198751000 \\ 6 & 3.614185000 & 0.195345000 & 0.041929000 \\ 6 & 2.786344000 & -0.453078000 & -1.034700000 \\ 6 & 1.935892000 & 0.585681000 & -0.979402000 \\ 6 & 0.657934000 & 0.931415000 & -1.575570000 \\ 1 & 0.353774000 & 0.310900000 & -2.411426000 \\ 1 & 0.608627000 & 1.977267000 & -1.915274000 \\ 17 & -1.065314000 & 2.343639000 & 0.479641000 \\ 17 & -0.447745000 & -2.037990000 & -1.064880000 \\ 8 & 2.537799000 & -1.050764000 & 1.846101000 \\ 1 & 2.117550000 & -1.611379000 & 1.190244000 \\ 1 & 2.765035000 & -1.425535000 & -1.497158000 \\ 7 & 2.577334000 & 1.278440000 & 0.075333000 \\ 1 & -0.162521000 & 1.738622000 & -0.638846000 \\ 1 & 2.897449000 & 2.222150000 & -0.105468000 \\ 1 & 4.592130000 & 0.561978000 & -0.278281000 \\ 1 & 4.145499000 & 0.110649000 & 2.109315000 \\ 79 & -0.884582000 & 0.029342000 & -0.157178000 \\ 17 & -2.410928000 & -0.869396000 & 1.330703000 \\ & & & \\ 19-\mathrm{Au} & & & \\ 6 & -1.676010000 & -1.657084000 & 1.564978000 \\ 1 & -2.482249000 & -2.132784000 & 2.139632000 \\ 6 & -2.271484000 & -1.281858000 & 0.232581000 \\ 6 & -3.257817000 & -0.154611000 & 0.113534000 \\ 6 & -2.498582000 & 0.498267000 & -0.760614000 \\ 6 & -2.538948000 & 1.681709000 & -1.626028000\end{array}$




$\begin{array}{lrrr}1 & -3.469235000 & 2.224655000 & -1.481560000 \\ 1 & -2.467468000 & 1.391620000 & -2.675991000 \\ 17 & 1.102869000 & -2.047529000 & -0.965627000 \\ 17 & -0.015342000 & 2.154581000 & 0.668284000 \\ 8 & -1.077955000 & -0.610428000 & 2.264247000 \\ 1 & -1.626013000 & 0.178271000 & 2.250639000 \\ 1 & -4.233481000 & 0.017388000 & 0.535046000 \\ 7 & -1.396216000 & -0.457931000 & -0.717095000 \\ 1 & -1.700856000 & 2.341580000 & -1.400001000 \\ 1 & -1.223518000 & -0.950129000 & -1.593145000 \\ 1 & -2.581548000 & -2.198276000 & -0.274994000 \\ 1 & -0.901650000 & -2.410050000 & 1.409004000 \\ 79 & 0.593530000 & 0.061118000 & -0.132532000 \\ 17 & 2.774315000 & 0.497644000 & 0.375014000\end{array}$

\begin{tabular}{|c|c|c|c|}
\hline \multicolumn{4}{|c|}{ TS2 5 NN-Au } \\
\hline 6 & 4.207151000 & -0.817038000 & -0.360609000 \\
\hline 1 & 3.851300000 & -1.515013000 & 0.407243000 \\
\hline 6 & 3.149057000 & 0.236564000 & -0.620344000 \\
\hline 6 & 1.813467000 & -0.360752000 & -0.981344000 \\
\hline 6 & 1.016919000 & -0.278686000 & 0.069435000 \\
\hline 6 & 1.751626000 & 0.267274000 & 1.262692000 \\
\hline 1 & 2.113411000 & -0.558487000 & 500000 \\
\hline 1 & 1.152304000 & 0.913305000 & 1.906184000 \\
\hline 17 & -0.411565000 & 2.380336000 & -0.133286000 \\
\hline 17 & -1.172121000 & -2.366155000 & 0.140806000 \\
\hline 8 & 5.371336000 & -0.141192000 & 0.054020000 \\
\hline 1 & 6.060938000 & -0.773292000 & 0.251818000 \\
\hline 1 & 1.563716000 & -0.718694000 & -1.968404000 \\
\hline 1 & 4.375538000 & -1.390278000 & -1.279050000 \\
\hline 1 & 3.521093000 & 0.905953000 & -1.400681000 \\
\hline 7 & 2.79 & 000 & 00 \\
\hline 1 & 3.61 & 566000 & 00 \\
\hline 1 & 0.892136000 & 2.213394000 & -0.062689000 \\
\hline 79 & -0.994918000 & -0.109816000 & 0.003967000 \\
\hline 17 & -3.283198000 & 0.461618000 & -0.118573000 \\
\hline & & & \\
\hline 6 & 4.081131000 & -0.555136000 & -0.701846000 \\
\hline 1 & 3.582617000 & -1.431860000 & -0.267067000 \\
\hline 6 & 3.238214000 & 0.675997000 & -0.417481000 \\
\hline 6 & 1.843679000 & 0.469304000 & -0.937244000 \\
\hline 6 & 1.0 & & \\
\hline 6 & 1.752804000 & 000 & 1000 \\
\hline 1 & 1.839354000 & -0.739835000 & 1.793773000 \\
\hline 1 & 1.225054000 & 0.873275000 & 2.143953000 \\
\hline 17 & -1.235520000 & 2.368682000 & -0.107233000 \\
\hline 17 & -0.438427000 & -2.308816000 & 0.105701000 \\
\hline 8 & 5.345531000 & -0.336253000 & -0.122016000 \\
\hline 1 & 5.880221000 & -1.123914000 & -0.209849000 \\
\hline 1 & 1.602449000 & 0.432979000 & -1.989703000 \\
\hline 1 & 4.155019000 & -0.710359000 & -1.784908000 \\
\hline 1 & 3.740005000 & 144000 & 000 \\
\hline 7 & 3.016296000 & 0.903972000 & 1.008400000 \\
\hline 1 & 3.806770000 & 0.602450000 & 1.559835000 \\
\hline 1 & 0.044158000 & 560936 & -0 \\
\hline
\end{tabular}




\begin{tabular}{lrrr}
79 & -0.967932000 & -0.103426000 & -0.010113000 \\
17 & -3.325777000 & -0.299624000 & -0.113176000 \\
\multicolumn{4}{c}{ TS3 } \\
5N-Au & & \\
6 & -3.866723000 & 0.257475000 & -1.080927000 \\
1 & -3.238593000 & 1.157070000 & -1.047675000 \\
6 & -3.280660000 & -0.810647000 & -0.143947000 \\
6 & -1.860053000 & -0.986561000 & -0.521384000 \\
6 & -1.026622000 & -0.374039000 & 0.386260000 \\
6 & -1.891596000 & 0.252179000 & 1.443596000 \\
1 & -1.881181000 & 1.333406000 & 1.265680000 \\
1 & -1.506449000 & 0.083211000 & 2.448311000 \\
17 & 1.295824000 & -2.289108000 & -0.050868000 \\
17 & 0.443984000 & 2.275803000 & 0.053964000 \\
8 & -5.159183000 & 0.504532000 & -0.601720000 \\
1 & -5.535141000 & 1.258316000 & -1.055702000 \\
1 & -1.510032000 & -1.531315000 & -1.390948000 \\
1 & -3.875190000 & -0.116190000 & -2.111023000 \\
1 & -3.879137000 & -1.718119000 & -0.258399000 \\
7 & -3.184692000 & -0.399201000 & 1.241782000 \\
1 & -3.982043000 & 0.149720000 & 1.528283000 \\
1 & -0.907093000 & -1.567753000 & 0.529669000 \\
79 & 0.978465000 & 0.022465000 & 0.030399000 \\
17 & 3.209390000 & 0.478281000 & -0.396628000
\end{tabular}

\section{5b-Au}

$\begin{array}{lccc}6 & 2.606859000 & -0.217628000 & -1.508183000 \\ 1 & 1.937030000 & -1.065432000 & -1.327706000 \\ 6 & 2.535112000 & 0.730420000 & -0.332391000 \\ 6 & 1.216336000 & 1.406296000 & -0.138333000 \\ 6 & 0.858384000 & 1.354250000 & 1.182743000 \\ 6 & 1.922974000 & 0.638135000 & 1.950858000 \\ 1 & 1.540635000 & -0.150633000 & 2.598125000 \\ 1 & 2.401415000 & 1.381562000 & 2.602946000 \\ 17 & -1.840992000 & 1.925193000 & -0.545760000 \\ 17 & 0.321295000 & -1.974707000 & 0.755174000 \\ 8 & 3.940886000 & -0.628008000 & -1.639580000 \\ 1 & 3.995394000 & -1.331425000 & -2.285065000 \\ 1 & 0.770698000 & 2.065863000 & -0.869616000 \\ 1 & 2.251867000 & 0.311173000 & -2.402146000 \\ 1 & 3.245414000 & 1.546680000 & -0.551639000 \\ 7 & 2.845731000 & 0.122255000 & 0.952838000 \\ 1 & 2.782122000 & -0.882925000 & 0.889457000 \\ 1 & 0.107964000 & 1.988085000 & 1.635709000 \\ 79 & -0.710116000 & -0.011288000 & 0.056169000 \\ 17 & -2.551982000 & -1.260592000 & -0.515813000 \\ & & & \\ 1 \mathrm{~b}-\mathrm{Pd}_{\mathrm{D}} & & & \\ 6 & -3.594741000 & 0.139487000 & -0.705894000 \\ 1 & -3.465901000 & 0.526748000 & -1.723027000 \\ 6 & -2.758347000 & -1.133248000 & -0.507757000 \\ 6 & -1.332944000 & -0.750872000 & -0.818383000 \\ 6 & -0.443000000 & -0.640767000 & 0.115775000 \\ 6 & 0.140698000 & -0.621627000 & 1.357110000 \\ 1 & 0.629342000 & -1.508620000 & 1.740569000 \\ 1 & -0.114766000 & 0.166991000 & 2.056598000\end{array}$




$\begin{array}{lrrr}17 & 0.320310000 & 2.308600000 & 0.077762000 \\ 17 & 2.640806000 & -1.592562000 & -0.268286000 \\ 8 & -3.292409000 & 1.104412000 & 0.263502000 \\ 1 & -2.425308000 & 1.486935000 & 0.087838000 \\ 1 & -1.109315000 & -0.425807000 & -1.827310000 \\ 7 & -2.887090000 & -1.679367000 & 0.826328000 \\ 1 & -3.034825000 & -0.924751000 & 1.486143000 \\ 1 & -3.676429000 & -2.303393000 & 0.889547000 \\ 1 & -3.083156000 & -1.879145000 & -1.236904000 \\ 1 & -4.647963000 & -0.114627000 & -0.576064000 \\ 46 & 1.414527000 & 0.299249000 & -0.047737000\end{array}$

\section{1b-Pdp}

$6 \quad 1.800416000$

$6 \quad 0.040708000$

$6-0.048295000$

$1-0.715948000$

10.560352000

$17-2.475840000$

$17-0.076867000$

$1 \quad 1.590939000$

$1 \quad 0.038701000$

$7 \quad 2.456748000$

13.430272000

$1 \quad 2.038928000$

$6 \quad 2.702708000$

$1 \quad 2.907721000$

12.206314000

$8 \quad 3.886397000$

$1 \quad 4.428106000$

$46-1.114840000$

0.827406000

$-0.395720000$

1.005861000

0.341183000

0.237077000

1.390155000

$-0.269658000$

2.580644000

$-1.085297000$

2.814368000

0.139459000

3.378467000

1.524633000

$-2.314197000$

0.490235000

$-0.265032000$

$-0.420091000$

1.988221000

$-1.419818000$

2.122683000

0.226666000

2.014140000

$-0.333297000$

2.783760000

$-0.585526000$

$-0.200988000$

$-0.972577000$

0.107589000

0.247132000

$-1.173512000$

1.280537000

$-0.224442000$

0.258313000

$-0.962089000$

$-0.517326000$

$-0.294377000$

$-0.242576000$

$-0.252162000$

TS1 4-exo-trig

$1-3.582281000$

$6-2.733029000$

$6-1.201883000$

$6-0.368409000$

$6-0.545376000$

$1 \quad 0.191337000$

$1-1.394254000$

$17 \quad 0.333715000$

$\begin{array}{ll}17 & 2.189301000\end{array}$

$8-1.516639000$

$1-0.928461000$

$1-0.742640000$

$7-3.352916000$

$1-3.467655000$

$1-4.222010000$

$1-3.127845000$

$\begin{array}{ll}1 & -2.701358000\end{array}$

$46 \quad 1.267463000$

TS1 $1_{5 \text {-exo-dig }}$
0.754125000

1.136191000

$-0.742566000$

$-0.891156000$

$-0.680017000$

$-1.083557000$

$-0.877200000$

$-1.701782000$

2.342500000

$-1.754250000$

0.872708000

1.531166000

$-1.371353000$

$-1.260448000$

$-0.577605000$

$-1.733989000$

$-1.268929000$

1.293470000

0.245006000
$-0.705911000$

$-1.299274000$

$-0.471783000$

$-0.511447000$

0.604967000

1.859760000

2.622629000

2.123710000

0.471876000

$-0.559298000$

$-1.414162000$

$-0.966533000$

$-1.369277000$

0.707644000

1.440325000

0.533052000

$-1.340531000$

0.244278000

0.026175000

0.740172000

$-0.332065000$ 


$\begin{array}{lccc}1 & -2.717318000 & 1.352798000 & -1.173703000 \\ 6 & -2.971988000 & -0.739500000 & -0.695937000 \\ 6 & -1.513073000 & -1.070691000 & -0.574757000 \\ 6 & -0.841979000 & -0.536220000 & 0.403963000 \\ 6 & 0.136176000 & -0.337809000 & 1.376028000 \\ 1 & 0.458927000 & -1.228993000 & 1.903569000 \\ 1 & 0.069160000 & 0.558786000 & 1.980883000 \\ 17 & 0.417005000 & 2.328138000 & -0.198516000 \\ 17 & 2.381000000 & -1.852414000 & -0.105520000 \\ 8 & -2.190529000 & 0.937694000 & 0.778518000 \\ 1 & -1.538036000 & 1.637876000 & 0.559305000 \\ 1 & -0.986821000 & -1.654454000 & -1.317672000 \\ 7 & -3.753042000 & -1.515414000 & 0.254247000 \\ 1 & -4.742851000 & -1.432111000 & 0.065532000 \\ 1 & -3.511620000 & -2.495143000 & 0.219054000 \\ 1 & -3.286330000 & -0.872320000 & -1.737626000 \\ 1 & -4.060548000 & 1.013531000 & -0.048484000 \\ 46 & 1.433728000 & 0.213116000 & -0.094641000 \\ & & & \\ \text { TS1 } & \text { 5-endo-dig } & & \\ 6 & -1.808614000 & 1.252300000 & -1.148633000 \\ 1 & -1.313332000 & 0.679230000 & -1.935657000 \\ 6 & -0.752840000 & 1.926991000 & -0.279042000 \\ 6 & -0.258890000 & 0.819406000 & 0.748808000 \\ 6 & -1.280575000 & 0.340451000 & 1.536302000 \\ 6 & -2.236495000 & -0.109011000 & 2.273252000 \\ 1 & -2.051161000 & -0.315613000 & 3.321567000 \\ 1 & -3.220995000 & -0.290556000 & 1.870377000 \\ 46 & 0.820565000 & -0.680009000 & -0.086640000 \\ 17 & -0.934438000 & -2.039767000 & -0.786918000 \\ 17 & 2.686203000 & 0.524544000 & 0.476148000 \\ 8 & -2.606228000 & 0.428012000 & -0.347473000 \\ 1 & -2.351825000 & -0.497574000 & -0.547407000 \\ 1 & 0.488586000 & 1.319999000 & 1.383250000 \\ 1 & -1.231907000 & 2.693603000 & 0.337894000 \\ 1 & -2.425182000 & 2.024495000 & -1.608975000 \\ 7 & 0.252534000 & 2.522543000 & -1.066021000 \\ 1 & 0.868650000 & 3.130037000 & -0.548157000 \\ 1 & 2.307726000 & -1.985973000 & 1.193241000 \\ 1 & 1.141685000 & 0.225371000 & -2.009076000 \\ 1 & 2.940409000 & 0.781593000 & 1.168978000 \\ 17 & 3.199456000 & -1.238129000 & -1.094274000\end{array}$




\begin{tabular}{|c|c|c|c|}
\hline 7 & 3.731023000 & 0.741819000 & -1.284064000 \\
\hline 1 & 3.865138000 & 0.593218000 & -2.272916000 \\
\hline 1 & 3.324752000 & 1.661988000 & -1.166280000 \\
\hline 46 & -1.443615000 & 0.170151000 & -0.099406000 \\
\hline \multicolumn{4}{|c|}{$\mathrm{TS}_{1 \text { 3-exo-trig }}$} \\
\hline 6 & -3.570574000 & -0.844635000 & -0.132775000 \\
\hline 1 & -3.325295000 & -1.404811000 & -1.046970000 \\
\hline 6 & -2.396885000 & 0.023385000 & 0.231826000 \\
\hline 6 & -1.107257000 & -0.658358000 & 0.322834000 \\
\hline 6 & -0.030966000 & -0.199459000 & 1.098671000 \\
\hline 6 & 0.018360000 & 0.090100000 & 2.391743000 \\
\hline 1 & 0.897594000 & 0.520433000 & 2.847789000 \\
\hline 1 & -0.812350000 & -0.154914000 & 3.045430000 \\
\hline 17 & 0.994703000 & 2.343029000 & 95782000 \\
\hline 17 & 1.789717000 & -2.186417000 & -0.347898000 \\
\hline 8 & -4.679704000 & -0.015168000 & -0.304414000 \\
\hline 1 & -5.443013000 & -0.537855000 & -0.546217000 \\
\hline 1 & -0.927091000 & -1.490823000 & -0.354373000 \\
\hline 7 & -1.945516000 & 0.848378000 & -0.884628000 \\
\hline 1 & -1.819565000 & 0.362898000 & -1.761615000 \\
\hline 1 & -1.198493000 & 1.516033000 & -0.688778000 \\
\hline 1 & -2.623760000 & 0.663110000 & 1.082486000 \\
\hline 1 & -3.71 & -1.5 & 2000 \\
\hline 46 & 1.417750000 & 0.068672000 & -0.192371000 \\
\hline \multicolumn{4}{|c|}{$\mathrm{TS}_{\text {4-exo-dig }}$} \\
\hline 6 & -3.60643 & 0.024020000 & -0.666560000 \\
\hline 1 & -3.556535000 & 0.416234000 & -1.687732000 \\
\hline 6 & -2.724196000 & -1.222054000 & -0.578221000 \\
\hline 6 & -1.311980000 & -0.906536000 & -0.900793000 \\
\hline 6 & -0.693202000 & -0.950439000 & 0.253331000 \\
\hline 6 & 0.268384000 & -0.741752000 & 1.240235000 \\
\hline 1 & 0.832971000 & 606000 & 1.572645000 \\
\hline 1 & -0.0204 & -0.04 & 000 \\
\hline 17 & 000 & 3000 & 5000 \\
\hline 17 & 2.856699000 & -1.332108000 & -0.296739000 \\
\hline 8 & -3.260515000 & 0.973238000 & 0.293219000 \\
\hline 1 & -2.399308000 & 1.377750000 & 0.097657000 \\
\hline 1 & -0.893839000 & -0.545169000 & -1.828559000 \\
\hline 7 & -2.484832000 & -1.574195000 & 0.843301000 \\
\hline 1 & -2.950554000 & -0.938237000 & 1.480295000 \\
\hline 1 & -2.636129000 & -2.541665000 & 1.084967000 \\
\hline 1 & -3.172806000 & -2.041412000 & -1.141291000 \\
\hline 1 & -4.642942000 & -0.258039000 & -0.471653000 \\
\hline 4 & 1.393206000 & 0.386028000 & -0.036477000 \\
\hline \multicolumn{4}{|c|}{ TS1 1-endo-dig } \\
\hline 6 & -3.14307 & 0.710002000 & -0.27460700 \\
\hline 1 & -3.193027000 & 1.311976000 & 0.643985000 \\
\hline 6 & -1.950514000 & -0.217189000 & -0.192913000 \\
\hline 6 & -0.703284000 & 0.566183000 & 0.132492000 \\
\hline 6 & -0.487483000 & 0.250408000 & 1.498153000 \\
\hline 6 & -0.007181000 & 0.219984000 & 2.701408000 \\
\hline 1 & 1.034030000 & 0.472708000 & 2.856212000 \\
\hline & -0. & -0.046751 & 3.5 \\
\hline
\end{tabular}




$\begin{array}{lrrr}17 & 0.410497000 & -2.371208000 & -0.539734000 \\ 17 & 2.033572000 & 1.906941000 & -0.047415000 \\ 8 & -2.981019000 & 1.501900000 & -1.412931000 \\ 1 & -3.651718000 & 2.182804000 & -1.438730000 \\ 1 & -0.680347000 & 1.616688000 & -0.143836000 \\ 1 & -1.891697000 & -0.830060000 & -1.090506000 \\ 1 & -4.055017000 & 0.098533000 & -0.324641000 \\ 7 & -2.045235000 & -1.013415000 & 1.045291000 \\ 1 & -2.987604000 & -1.187213000 & 1.373157000 \\ 1 & -1.535592000 & -1.887191000 & 0.934105000 \\ 46 & 1.129289000 & -0.172535000 & -0.338624000\end{array}$

TS1 $1_{\text {5-endo-trig }}$

$\begin{array}{lrrr}6 & -3.447285000 & 0.498415000 & -0.495649000 \\ 1 & -2.894633000 & 1.266334000 & 0.061801000 \\ 6 & -2.634732000 & -0.778492000 & -0.477981000 \\ 6 & -1.274044000 & -0.501456000 & -1.083052000 \\ 6 & -0.332636000 & -0.334765000 & -0.192219000 \\ 6 & -0.375745000 & -0.451912000 & 1.198654000 \\ 1 & -0.553889000 & 0.422408000 & 1.813235000 \\ 1 & -0.022975000 & -1.352111000 & 1.684478000 \\ 17 & 2.288739000 & -1.926540000 & -0.149866000 \\ 17 & 0.912788000 & 2.419743000 & 0.136062000 \\ 8 & -4.697059000 & 0.223544000 & 0.092031000 \\ 1 & -5.213329000 & 1.026944000 & 0.143932000 \\ 1 & -1.166249000 & -0.370720000 & -2.148306000 \\ 1 & -3.545890000 & 0.842911000 & -1.529489000 \\ 1 & -3.164856000 & -1.541632000 & -1.055798000 \\ 7 & -2.465059000 & -1.204472000 & 0.910086000 \\ 1 & -3.262883000 & -0.927664000 & 1.469472000 \\ 1 & -2.346888000 & -2.204807000 & 0.985126000 \\ 46 & 1.542700000 & 0.228393000 & -0.043373000\end{array}$

\begin{tabular}{|c|c|c|c|}
\hline $1_{4-}$ & o-trig & & \\
\hline 6 & -2.862091000 & 0.765287000 & -0.570698000 \\
\hline 1 & -3.563207000 & 1.246818000 & -1.245022000 \\
\hline 6 & -2.844245000 & -0.757419000 & -0.460134000 \\
\hline 6 & -1.305884000 & -0.678836000 & -0.713241000 \\
\hline 6 & -0.330749000 & -0.788703000 & 0.389686000 \\
\hline 6 & -0.402352000 & -1.631708000 & 1.401948000 \\
\hline 1 & 0.424437000 & -1.756267000 & 2.085605000 \\
\hline 1 & -1.275690000 & -2.255285000 & 1.549438000 \\
\hline 17 & 0.114300000 & 2.338139000 & 0.495498000 \\
\hline 17 & 2.424814000 & -1.550259000 & -0.550276000 \\
\hline 8 & -1.516883000 & 0.765069000 & -1.147462000 \\
\hline 1 & -0.865796000 & 1.439335000 & 88000 \\
\hline 1 & -0.945990000 & -1.207623000 & -1.594014000 \\
\hline 7 & -3.332362000 & -1.399669000 & 0.709541000 \\
\hline 1 & -3.118896000 & -0.904314000 & 1.562584000 \\
\hline 1 & -4.315675000 & -1.608470000 & 0.663185000 \\
\hline 1 & -3.314193000 & -1.215173000 & -1.328654000 \\
\hline 1 & -2.829068000 & 1.268287000 & 0.397313000 \\
\hline 46 & 1.273319000 & 0.300525000 & 0.073822000 \\
\hline & 2.702212000 & 0.784698000 & -0.575279000 \\
\hline
\end{tabular}




$\begin{array}{lrrr}1 & -2.194887000 & 1.112253000 & -1.484233000 \\ 6 & -3.118437000 & -0.679944000 & -0.640719000 \\ 6 & -1.902072000 & -1.342100000 & -0.075529000 \\ 6 & -1.140823000 & -0.468045000 & 0.569341000 \\ 6 & 0.123761000 & -0.546259000 & 1.258314000 \\ 1 & 0.411494000 & -1.565140000 & 1.487685000 \\ 1 & 0.216699000 & 0.123992000 & 2.109677000 \\ 17 & 0.478096000 & 2.376274000 & -0.051555000 \\ 17 & 2.398887000 & -1.877491000 & -0.267013000 \\ 8 & -1.712744000 & 0.831594000 & 0.494804000 \\ 1 & -0.852855000 & 1.601410000 & 0.282313000 \\ 1 & -1.683291000 & -2.394680000 & -0.145615000 \\ 7 & -4.288894000 & -0.938110000 & 0.188690000 \\ 1 & -5.146017000 & -0.927680000 & -0.344245000 \\ 1 & -4.220862000 & -1.827356000 & 0.660254000 \\ 1 & -3.275765000 & -0.964514000 & -1.685625000 \\ 1 & -3.510306000 & 1.455848000 & -0.303924000 \\ 46 & 1.467609000 & 0.181097000 & -0.079493000\end{array}$

$\begin{array}{lccc}\text { I1 } & \\ \text { 5-endo-dig } & & & \\ 6 & -1.901735000 & 1.309209000 & -0.927127000 \\ 1 & -1.704732000 & 0.771110000 & -1.853405000 \\ 6 & -0.628019000 & 1.933400000 & -0.360889000 \\ 6 & -0.219746000 & 0.945811000 & 0.730231000 \\ 6 & -1.482603000 & 0.405198000 & 1.196569000 \\ 6 & -1.933654000 & 0.000819000 & 2.368533000 \\ 1 & -2.940220000 & -0.368548000 & 2.487837000 \\ 1 & -1.277637000 & 0.023175000 & 3.224131000 \\ 46 & 0.783895000 & -0.656960000 & -0.065901000 \\ 17 & -1.126980000 & -1.914050000 & -0.888584000 \\ 17 & 2.721906000 & 0.385897000 & 0.493221000 \\ 8 & -2.346222000 & 0.317683000 & 0.058821000 \\ 1 & -2.020810000 & -0.728800000 & -0.394877000 \\ 1 & 0.431120000 & 1.340607000 & 1.501400000 \\ 1 & -0.893280000 & 2.863991000 & 0.153076000 \\ 1 & -2.717907000 & 2.009554000 & -1.070244000 \\ 7 & 0.281201000 & 2.276114000 & -1.421162000 \\ 1 & 1.054405000 & 2.816930000 & -1.058073000 \\ 1 & 0.692068000 & 1.429864000 & -1.803591000 \\ \mathbf{I} & & & \\ 6 & 3 . \text {-endo-trig } & & \\ 1 & 4.147212000 & -0.520223000 & 0.645858000 \\ 6 & 2.847089000 & -0.964411000 & 0.726410000 \\ 6 & 1.370852000 & 0.260620000 & -0.732330000 \\ 6 & 0.463900000 & -0.156090000 & -0.023949000 \\ 6 & 0.739378000 & -0.981468000 & 1.149111000 \\ 1 & 0.121431000 & -1.875540000 & 1.203057000 \\ 1 & 0.760769000 & -0.446175000 & 2.096586000 \\ 17 & -1.108527000 & 2.378604000 & 0.501935000 \\ 17 & -1.829666000 & -2.047810000 & -0.546988000 \\ 8 & 2.189922000 & -1.562997000 & 1.046013000 \\ 1 & 2.156770000 & -2.254108000 & 0.367190000 \\ 1 & 1.080530000 & 0.855594000 & -1.749080000 \\ 1 & 3.036650000 & 0.252438000 & 1.402547000 \\ 1 & 3.201029000 & -0.700151000 & -1.482885000\end{array}$




$\begin{array}{llcc}7 & 3.676600000 & 1.203166000 & -0.856500000 \\ 1 & 3.858076000 & 1.428182000 & -1.822863000 \\ 1 & 3.209676000 & 1.999643000 & -0.439531000 \\ 46 & -1.442319000 & 0.183649000 & -0.060611000\end{array}$

I1 $1_{\text {3-exo-trig }}$

$\begin{array}{lrrr}6 & -3.763764000 & -0.937148000 & -0.159110000 \\ 1 & -3.533355000 & -1.539172000 & -1.048859000 \\ 6 & -2.511719000 & -0.301728000 & 0.344720000 \\ 6 & -1.180265000 & -0.805808000 & -0.039584000 \\ 6 & -0.005134000 & -0.603481000 & 0.826895000 \\ 6 & 0.032143000 & -0.984392000 & 2.087299000 \\ 1 & 0.922257000 & -0.888280000 & 2.690751000 \\ 1 & -0.839359000 & -1.427916000 & 2.561128000 \\ 17 & 0.410591000 & 2.382457000 & 0.149006000 \\ 17 & 2.379163000 & -1.749841000 & -0.587265000 \\ 8 & -4.668145000 & 0.092492000 & -0.446934000 \\ 1 & -5.526672000 & -0.272547000 & -0.660348000 \\ 1 & -1.128950000 & -1.637618000 & -0.734015000 \\ 7 & -1.728432000 & 0.424228000 & -0.670272000 \\ 1 & -2.046225000 & 0.367917000 & -1.628497000 \\ 1 & -1.193066000 & 1.293599000 & -0.409512000 \\ 1 & -2.579053000 & 0.251438000 & 1.272836000 \\ 1 & -4.135747000 & -1.620940000 & 0.609991000 \\ 46 & 1.448934000 & 0.278367000 & -0.115152000\end{array}$

$\begin{array}{lrrr}\text { I1 } & \text { 4-exo-dig } \\ 6 & -2.985860000 & 0.124521000 & -1.051219000 \\ 1 & -3.759658000 & -0.637235000 & -0.900548000 \\ 6 & -2.735189000 & 0.786880000 & 0.298304000 \\ 6 & -2.169921000 & -0.091610000 & 1.366009000 \\ 6 & -0.955823000 & 0.452760000 & 1.294885000 \\ 6 & 0.408612000 & 0.225981000 & 1.678414000 \\ 1 & 0.511273000 & -0.624083000 & 2.344932000 \\ 1 & 0.958506000 & 1.104801000 & 2.001282000 \\ 17 & 1.725700000 & 1.953924000 & -0.486860000 \\ 17 & 0.286672000 & -2.398915000 & -0.008212000 \\ 8 & -1.824872000 & -0.377838000 & -1.622477000 \\ 1 & -1.448264000 & -1.126869000 & -1.127538000 \\ 1 & -2.562576000 & -0.947041000 & 1.886405000 \\ 7 & -1.369806000 & 1.486344000 & 0.290249000 \\ 1 & -0.833047000 & 1.395681000 & -0.585441000 \\ 1 & -1.334482000 & 2.450847000 & 0.609074000 \\ 1 & -3.541760000 & 1.471886000 & 0.564229000 \\ 1 & -3.389121000 & 0.860839000 & -1.749071000 \\ 46 & 1.217511000 & -0.277324000 & -0.112987000 \\ & & & \\ \mathbf{I} \mathbf{1}_{\text {4-endo-dig }} & 3.082962000 & -0.650201000 & -0.468691000 \\ 6 & 3.266073000 & -1.054507000 & 0.536772000 \\ 1 & 1.779597000 & 0.111882000 & -0.469957000 \\ 6 & 0.637082000 & -0.589353000 & 0.264250000 \\ 6 & 1.001517000 & 0.180460000 & 1.463691000 \\ 6 & 0.893060000 & 0.162875000 & 2.774602000 \\ 6 & 0.308341000 & -0.613946000 & 3.244109000 \\ 1 & 1.344744000 & 0.918949000 & 3.399003000 \\ 1 & & & \end{array}$




$\begin{array}{llrr}17 & -0.572694000 & 2.349730000 & -0.651860000 \\ 17 & -2.025577000 & -1.980126000 & 0.186909000 \\ 8 & 2.940614000 & -1.656499000 & -1.423182000 \\ 1 & 3.684892000 & -2.255784000 & -1.386244000 \\ 1 & 0.602112000 & -1.672171000 & 0.282094000 \\ 1 & 1.572112000 & 0.562783000 & -1.436596000 \\ 1 & 3.906608000 & 0.035944000 & -0.713149000 \\ 7 & 1.786980000 & 1.151372000 & 0.644347000 \\ 1 & 2.680458000 & 1.477398000 & 1.000162000 \\ 1 & 1.150199000 & 1.929605000 & 0.333485000 \\ 46 & -1.190087000 & 0.093193000 & -0.257909000\end{array}$

I1 5-endo-trig

$\begin{array}{lrrr}6 & -3.357295000 & 0.540308000 & -0.477847000 \\ 1 & -2.767056000 & 1.300533000 & 0.046517000 \\ 6 & -2.506326000 & -0.691626000 & -0.690773000 \\ 6 & -1.125536000 & -0.371933000 & -1.169133000 \\ 6 & -0.270231000 & -0.324457000 & -0.157016000 \\ 6 & -0.903498000 & -0.708253000 & 1.130317000 \\ 1 & -1.089880000 & 0.157545000 & 1.767459000 \\ 1 & -0.353985000 & -1.463649000 & 1.686721000 \\ 17 & 2.250309000 & -1.931407000 & -0.079256000 \\ 17 & 0.782668000 & 2.425854000 & 0.193159000 \\ 8 & -4.485876000 & 0.137230000 & 0.269566000 \\ 1 & -5.039167000 & 0.892840000 & 0.466230000 \\ 1 & -0.928399000 & -0.102458000 & -2.193711000 \\ 1 & -3.627020000 & 0.944259000 & -1.456769000 \\ 1 & -3.059869000 & -1.432792000 & -1.269244000 \\ 7 & -2.234883000 & -1.290420000 & 0.674785000 \\ 1 & -3.023563000 & -1.090714000 & 1.291865000 \\ 1 & -2.135972000 & -2.296386000 & 0.583233000 \\ 46 & 1.542887000 & 0.259915000 & -0.033738000\end{array}$

TS2 6

$\begin{array}{lrrr}6 & -3.142436000 & -0.375768000 & 0.663451000 \\ 1 & -3.064751000 & -1.414536000 & 0.308633000 \\ 6 & -2.815711000 & 0.554421000 & -0.490953000 \\ 6 & -1.309520000 & 0.566648000 & -0.698729000 \\ 6 & -0.501435000 & 0.012125000 & 0.189060000 \\ 6 & -0.971555000 & -0.575990000 & 1.476702000 \\ 1 & -0.891456000 & -1.669848000 & 1.409461000 \\ 1 & -0.347823000 & -0.256068000 & 2.311720000 \\ 17 & 1.492469000 & 2.379478000 & 0.148459000 \\ 17 & 1.365290000 & -2.308739000 & -0.349027000 \\ 8 & -2.286144000 & -0.168378000 & 1.755585000 \\ 46 & 1.439348000 & -0.068038000 & -0.103880000 \\ 1 & 0.191237000 & 2.333901000 & 0.115293000 \\ 1 & -0.950650000 & 0.961882000 & -1.644064000 \\ 1 & -4.161928000 & -0.212488000 & 1.006703000 \\ 7 & -3.564672000 & 0.122775000 & -1.651617000 \\ 1 & -3.506538000 & 0.786171000 & -2.410018000 \\ 1 & -3.222813000 & -0.767903000 & -1.989991000 \\ 1 & -3.151390000 & 1.565053000 & -0.240163000 \\ \mathbf{I}_{6} & & & \\ 6 & -3.142436000 & -0.375768000 & 0.663451000\end{array}$




$\begin{array}{lccc}1 & -3.064751000 & -1.414536000 & 0.308633000 \\ 6 & -2.815711000 & 0.554421000 & -0.490953000 \\ 6 & -1.309520000 & 0.566648000 & -0.698729000 \\ 6 & -0.501435000 & 0.012125000 & 0.189060000 \\ 6 & -0.971555000 & -0.575990000 & 1.476702000 \\ 1 & -0.891456000 & -1.669848000 & 1.409461000 \\ 1 & -0.347823000 & -0.256068000 & 2.311720000 \\ 17 & 1.492469000 & 2.379478000 & 0.148459000 \\ 17 & 1.365290000 & -2.308739000 & -0.349027000 \\ 8 & -2.286144000 & -0.168378000 & 1.755585000 \\ 46 & 1.439348000 & -0.068038000 & -0.103880000 \\ 1 & 0.191237000 & 2.333901000 & 0.115293000 \\ 1 & -0.950650000 & 0.961882000 & -1.644064000 \\ 1 & -4.161928000 & -0.212488000 & 1.006703000 \\ 7 & -3.564672000 & 0.122775000 & -1.651617000 \\ 1 & -3.506538000 & 0.786171000 & -2.410018000 \\ 1 & -3.222813000 & -0.767903000 & -1.989991000 \\ 1 & -3.151390000 & 1.565053000 & -0.240163000\end{array}$

$\mathrm{TS}_{6 \mathrm{~B}}$

\begin{tabular}{|c|c|c|c|}
\hline 6 & -4.522086000 & -0.744031000 & 1.162039000 \\
\hline 1 & -4.236294000 & -1.802780000 & 1.065539000 \\
\hline 6 & -3.089876000 & 0.097740000 & -0.656768000 \\
\hline 6 & -2.097411000 & 0.016069000 & 0.206405000 \\
\hline 6 & -2.299688000 & -0.252293000 & 1.658726000 \\
\hline 1 & -1.953593000 & -1.271965000 & 1.882800000 \\
\hline 1 & -1.715127000 & 0.432567000 & 2.277509000 \\
\hline 17 & -0.504156000 & 2.771247000 & 0.027063000 \\
\hline 17 & -0.175486000 & -2.003507000 & -0.601614000 \\
\hline 8 & -3.643669000 & -0.080051000 & 2.032243000 \\
\hline 1 & -5.049041000 & 0.838928000 & -0.181006000 \\
\hline 46 & -0.198976000 & 0.278319000 & -0.347267000 \\
\hline 1 & 0.134810000 & 2.905521000 & 1.135411000 \\
\hline 6 & 2.249143000 & 0.578431000 & -0.399861000 \\
\hline 1 & 2.363719000 & 1.515043000 & 0.136646000 \\
\hline 6 & 1.790397000 & 0.590180000 & -1.663441000 \\
\hline 1 & 1.538873000 & 1.517907000 & -2.158998000 \\
\hline 1 & 1.774637000 & -0.311633000 & -2.260630000 \\
\hline 6 & 2.795177000 & -0.620850000 & 0.273983000 \\
\hline 1 & 2.613999000 & -1.531572000 & -0.286293000 \\
\hline 35 & 4.735768000 & -0.406691000 & 0.444451000 \\
\hline 1 & 2.427227000 & -0.741046000 & 1.288440000 \\
\hline 6 & -4.525293000 & -0.120749000 & -0.222154000 \\
\hline 1 & 516199000 & 909000 & 98000 \\
\hline 7 & -5.279223000 & -0.990979000 & -1.105615000 \\
\hline 1 & -5.433728000 & -0.560714000 & -2.006069000 \\
\hline 1 & -4.770672000 & -1.852436000 & -1.264758000 \\
\hline 1 & -2.921968000 & 0.254308000 & -1.718034000 \\
\hline \multicolumn{4}{|c|}{$13_{6 \mathrm{Br}}$} \\
\hline 6 & 4.766039000 & -0.034531000 & 0.53303200 \\
\hline 1 & 4.948825000 & 0.246061000 & -0.516105000 \\
\hline 6 & 2.534828000 & -0.981527000 & 0.186009000 \\
\hline 6 & 2.118788000 & 0.264446000 & 0.187548000 \\
\hline 6 & 2.936697000 & 1.433585000 & 0.57380100 \\
\hline 1 & 3.166577000 & 2.035317000 & -0.31545600 \\
\hline
\end{tabular}




\begin{tabular}{|c|c|c|c|}
\hline 1 & 2.400880000 & 2.078779000 & 1.2672 \\
\hline 17 & -0.009968000 & 2.301741000 & -0.483362000 \\
\hline 8 & 4.114825000 & 0.997442000 & 1.215564000 \\
\hline 1 & 3.988063000 & -1.758559000 & 1.554609000 \\
\hline 46 & 0.267104000 & 0.018530000 & -0.412672000 \\
\hline 6 & -1.936383000 & -0.785980000 & -0.424533000 \\
\hline 1 & -1.824716000 & -1.786741000 & -0.018465000 \\
\hline 6 & -1.584550000 & -0.543672000 & -1.707869000 \\
\hline 1 & -1.203396000 & -1.332058000 & -2.342267000 \\
\hline 1 & -1.821671000 & 0.399760000 & -2.182011000 \\
\hline 6 & -2.687418000 & 0.175760000 & 0.415023000 \\
\hline 1 & -2.711857000 & 1.171140000 & -0.016483000 \\
\hline 35 & -4.544477000 & -0.436613000 & 12000 \\
\hline 1 & -2.318794000 & 0.227330000 & 1.434519000 \\
\hline 6 & 3.972460000 & -1.336042000 & 0.546467000 \\
\hline 1 & 5.731505000 & -0.187798000 & 1.011987000 \\
\hline 7 & 4.613516000 & -2.275111000 & -0.339940000 \\
\hline 1 & 4.235622000 & -3.206949000 & -0.259537000 \\
\hline 1 & 4.540393000 & -1.980809000 & -1.304940000 \\
\hline 1 & 1.871972000 & -1.811999000 & -0.104227000 \\
\hline \multicolumn{4}{|c|}{ TS4 $_{6 \mathrm{Br}}$} \\
\hline 6 & -4.274526000 & -0.918987000 & 0000 \\
\hline 1 & 000 & 00 & 000 \\
\hline 6 & 000 & 000 & 00 \\
\hline 6 & -1.578638000 & -0.499903000 & -0.275451000 \\
\hline 6 & -2.301857000 & -0.517314000 & -1.590657000 \\
\hline 1 & -2.538665000 & 0.511406000 & -1.899748000 \\
\hline 1 & -1.677184000 & -0.946402000 & -2.375276000 \\
\hline 17 & 0.650219000 & 3.118290000 & 0.139491000 \\
\hline 8 & -3.471159000 & -1.286522000 & -1.525479000 \\
\hline 1 & -3.705323000 & -2.371108000 & 1.028158000 \\
\hline 40 & -0.215734000 & 000 & -0.0 \\
\hline 6 & 000 & -0.1 & 000 \\
\hline 1 & 1.736016000 & 0.177215000 & -1.581477000 \\
\hline 6 & 0.384383000 & -1.059245000 & -0.419941000 \\
\hline 1 & 0.089023000 & -1.616523000 & -1.299451000 \\
\hline 1 & 0.410289000 & -1.655446000 & 0.484682000 \\
\hline 0 & 2.480457000 & 0.065309000 & 0.461115000 \\
\hline 1 & 2.110766000 & -0.107838000 & 1.466915000 \\
\hline 35 & 3.862108000 & -1.297849000 & 0.146072000 \\
\hline 1 & 2.977061000 & 1.026326000 & 0.404446000 \\
\hline 6 & -3.621983000 & -1.2 & 000 \\
\hline 1 & -5.2 & 3049000 & -0.541228000 \\
\hline 1 & -1.606398000 & -1.011163000 & 1.772469000 \\
\hline 7 & -4.360908000 & -0.651264000 & 1.958173000 \\
\hline 1 & -4.076368000 & -0.990152000 & 2.865634000 \\
\hline 1 & -4.219546000 & 0.351060000 & 1.945018000 \\
\hline \multicolumn{4}{|c|}{$14_{6 \mathrm{Br}}$} \\
\hline 6 & -3.613556000 & -0.844212000 & -0.228821000 \\
\hline 1 & -3.441534000 & 0.108981000 & -0.755260000 \\
\hline 6 & -1.453378000 & -0.946232000 & 0.958990000 \\
\hline 6 & -0.909234000 & -1.361719000 & -0.227403000 \\
\hline 6 & -1.796693000 & -1.798755000 & -1.355804000 \\
\hline & -1.679857000 & -1.121768000 & -2.215859000 \\
\hline
\end{tabular}




$\begin{array}{lccc}1 & -1.468252000 & -2.788330000 & -1.685211000 \\ 17 & 0.061089000 & 2.936946000 & -0.615095000 \\ 8 & -3.139092000 & -1.923328000 & -0.98845800 \\ 1 & -3.307056000 & -1.644273000 & 1.720927000 \\ 46 & -0.636258000 & 0.833196000 & -0.098139000 \\ 6 & 1.119244000 & -0.157733000 & -0.478630000 \\ 1 & 1.352902000 & 0.178896000 & -1.485966000 \\ 6 & 0.587049000 & -1.562299000 & -0.378784000 \\ 1 & 0.816089000 & -2.151458000 & -1.267318000 \\ 1 & 0.998267000 & -2.089602000 & 0.484220000 \\ 6 & 2.104108000 & 0.295767000 & 0.531584000 \\ 1 & 1.815359000 & 0.059917000 & 1.551924000 \\ 35 & 3.782743000 & -0.699512000 & 0.224921000 \\ 1 & 2.350488000 & 1.346867000 & 0.438048000 \\ 6 & -2.941542000 & -0.797115000 & 1.132345000 \\ 1 & -4.687891000 & -0.968148000 & -0.114752000 \\ 1 & -0.843402000 & -0.913022000 & 1.858508000 \\ 7 & -3.355224000 & 0.416000000 & 1.812130000 \\ 1 & -2.992698000 & 0.468825000 & 2.753331000 \\ 1 & -3.055744000 & 1.249334000 & 1.318080000 \\ 6 & & & \\ 6 \mathbf{b}-\mathbf{P d} & & & \\ 6 & 3.268224000 & -0.911255000 & -0.334964000 \\ 1 & 2.572052000 & -1.596009000 & -0.827147000 \\ 6 & 1.690184000 & 0.565330000 & 0.907685000 \\ 6 & 1.506534000 & 1.276969000 & -0.238610000 \\ 6 & 2.316644000 & 0.909727000 & -1.452694000 \\ 1 & 1.697818000 & 0.331808000 & -2.155195000 \\ 1 & 2.614559000 & 1.827711000 & -1.963314000 \\ 17 & -0.006792000 & -2.031611000 & -0.675532000 \\ 8 & 3.492456000 & 0.233904000 & -1.128812000 \\ 1 & 3.544451000 & -0.062364000 & 1.593587000 \\ 46 & -0.507962000 & 0.070622000 & 0.109945000 \\ 6 & -0.731295000 & 2.250003000 & 0.021994000 \\ 1 & -1.464741000 & 2.384810000 & -0.763699000 \\ 6 & 0.704579000 & 2.566350000 & -0.290070000 \\ 1 & 0.779899000 & 3.000252000 & -1.285349000 \\ 1 & 1.119389000 & 3.292938000 & 0.411433000 \\ 6 & -1.165658000 & 1.891122000 & 1.266291000 \\ 1 & -0.517619000 & 1.944008000 & 2.130374000 \\ 1 & -2.219156000 & 1.753913000 & 1.459151000 \\ 6 & 2.724500000 & -0.525089000 & 1.029558000 \\ 1 & 4.224834000 & -1.415034000 & -0.218851000 \\ 1 & 1.308474000 & 0.940169000 & 1.852069000 \\ 7 & 2.325561000 & -1.713994000 & 1.745161000 \\ 1 & 2.120200000 & -1.528140000 & 2.715382000 \\ 1 & 1.512098000 & -2.137158000 & 1.310843000 \\ 35 & -2.854611000 & -0.441724000 & -0.184956000 \\ & & & \end{array}$

$\mathrm{TS}_{5}$

$\begin{array}{rrrr}6 & 2.803228000 & -1.842538000 & -0.477660000 \\ 1 & 2.131626000 & -2.480901000 & 0.109589000 \\ 6 & 2.187444000 & -0.470561000 & -0.624755000 \\ 6 & 0.765830000 & -0.511486000 & -1.107005000 \\ 6 & -0.039908000 & -0.222179000 & -0.101973000 \\ 6 & 0.670822000 & -0.067259000 & 1.202534000\end{array}$




$\begin{array}{lccc}1 & 0.603021000 & -0.971667000 & 1.809505000 \\ 1 & 0.350684000 & 0.796606000 & 1.782976000 \\ 17 & 1.452744000 & 2.895105000 & 0.127046000 \\ 17 & -2.525558000 & -1.635665000 & 0.537476000 \\ 8 & 4.044971000 & -1.669488000 & 0.159836000 \\ 1 & 4.470606000 & -2.516278000 & 0.288319000 \\ 1 & 0.500975000 & -0.714849000 & -2.131802000 \\ 1 & 2.905377000 & -2.289579000 & -1.471592000 \\ 1 & 2.826456000 & 0.167115000 & -1.239590000 \\ 7 & 2.046539000 & 0.189927000 & 0.704414000 \\ 1 & 2.795604000 & -0.074404000 & 1.336906000 \\ 1 & 2.005569000 & 1.336666000 & 0.508206000 \\ 46 & -1.855698000 & 0.348856000 & -0.257518000\end{array}$

$12_{5}$

6

1

6

6

6

6

$1 \quad 0.346006000$

$1 \quad 0.416738000$

$17 \quad 2.302555000$

$17-2.615719000$

$8 \quad 3.707400000$

13.988202000

$1 \quad 0.357469000$

$1 \quad 2.490713000$

12.788575000

$7 \quad 2.003092000$

$1 \quad 2.659108000$

$1 \quad 2.264108000$

$46-1.938955000$

\section{TS4 ${ }_{5 \mathrm{Br}}$}

$6 \quad 1.731527000$

$6 \quad 1.396694000$

$6 \quad 2.384408000$

$17-0.786521000$

$46 \quad 0.115084000$

$6-1.583554000$

$1-2.066649000$

$6-0.500284000$

$1-0.222464000$

$1-0.427615000$

$6-2.404192000$

$1-1.845939000$

$35-3.845573000$

$1-2.881600000$

$1 \quad 1.840576000$

12.389036000

$1 \quad 0.939330000$

$7 \quad 2.961472000$

$6 \quad 3.554176000$

13.978081000

-2.102885000
-2.642884000
-0.646496000
-0.491398000
-0.093451000
0.015353000
-0.819581000
0.953461000
2.740094000
-1.405149000
-2.141529000
-3.048011000
-0.717677000
-2.536613000
-0.102556000
-0.007254000
-0.437675000
1.241207000
0.489403000

$-0.521980000$

0.051678000

$-0.627007000$

$-1.122826000$

$-0.126526000$

1.175687000

1.832699000

1.701478000

0.166981000

0.683810000

0.116535000

0.235279000

$-2.136113000$

$-1.526872000$

$-1.230041000$

0.709972000

1.351887000

0.515571000

$-0.300548000$

$-1.161582000$

$-0.440688000$

$-0.537522000$

3.156709000

1.120323000

0.081014000

0.663238000

$-0.775742000$

$-0.838508000$

$-1.715666000$

$-0.177981000$

$-0.670392000$

$-1.414070000$

0.719282000

$-0.446068000$

$-0.155704000$

$-1.858777000$

$-1.858568000$

$-1.234813000$

$-2.000078000$
$-1.045040000$

0.227782000

1.111852000

$-0.392186000$

0.129166000

0.635591000

1.413034000

0.989292000

2.031911000

0.452913000

$-0.570765000$

$-1.361986000$

$-0.071896000$

$-0.947579000$

$-1.872017000$

2.122341000

$-1.342142000$

$-0.694209000$

0.487268000

1.148016000 


$\begin{array}{rrrr}1 & 3.624963000 & -1.939645000 & -1.449947000 \\ 6 & 4.661655000 & -0.252812000 & 0.152338000 \\ 1 & 5.015532000 & 0.230541000 & 1.071064000 \\ 1 & 4.255120000 & 0.526681000 & -0.506182000 \\ 8 & 5.688771000 & -0.978372000 & -0.480873000 \\ 1 & 6.390603000 & -0.383684000 & -0.741022000\end{array}$

$\begin{array}{lccc}\mathbf{I 4}_{5 \mathrm{Br}} & & & \\ 6 & 1.074865000 & -2.073896000 & -0.845330000 \\ 6 & 0.625824000 & -1.424495000 & 0.427672000 \\ 6 & 1.707367000 & -0.966842000 & 1.124628000 \\ 17 & -0.012888000 & 2.902315000 & -0.301289000 \\ 46 & 0.624683000 & 0.780095000 & 0.238970000 \\ 6 & -1.231996000 & -0.013305000 & 0.659876000 \\ 1 & -1.553174000 & 0.559711000 & 1.525602000 \\ 6 & -0.794101000 & -1.432616000 & 0.926806000 \\ 1 & -0.833258000 & -1.679578000 & 1.986578000 \\ 1 & -1.406003000 & -2.157649000 & 0.385992000 \\ 6 & -2.069501000 & 0.215967000 & -0.544131000 \\ 1 & -1.701381000 & -0.299780000 & -1.427081000 \\ 35 & -3.858028000 & -0.549781000 & -0.199582000 \\ 1 & -2.226229000 & 1.265620000 & -0.757538000 \\ 1 & 0.590236000 & -1.643386000 & -1.727293000 \\ 1 & 1.705267000 & -0.709452000 & 2.175788000 \\ 1 & 0.780170000 & -3.133261000 & -0.817565000 \\ 7 & 2.515782000 & -1.906343000 & -0.848581000 \\ 6 & 2.971711000 & -1.220917000 & 0.354710000 \\ 1 & 3.644729000 & -1.863191000 & 0.939440000 \\ 1 & 2.908575000 & -1.528155000 & -1.694217000 \\ 6 & 3.722494000 & 0.059339000 & 0.053862000 \\ 1 & 4.025981000 & 0.532431000 & 0.995321000 \\ 1 & 3.048242000 & 0.761176000 & -0.470403000 \\ 8 & 4.824255000 & -0.266648000 & -0.750974000 \\ 1 & 5.287025000 & 0.532288000 & -1.000650000\end{array}$

\begin{tabular}{lccc}
\multicolumn{3}{l}{ 7b-Pd } \\
6 & 1.953799000 & 1.242651000 & 1.557927000 \\
6 & 1.100340000 & 1.617000000 & 0.376138000 \\
6 & 1.576775000 & 0.966940000 & -0.722841000 \\
17 & 0.230553000 & -1.873369000 & 0.848517000 \\
46 & -0.554419000 & 0.071582000 & -0.107608000 \\
6 & -1.204811000 & 2.172999000 & -0.076244000 \\
1 & -2.043518000 & 2.165540000 & 0.608982000 \\
6 & 0.102720000 & 2.746410000 & 0.402424000 \\
1 & 0.432762000 & 3.577919000 & -0.223405000 \\
1 & 0.002712000 & 3.121342000 & 1.419419000 \\
6 & -1.399986000 & 1.738370000 & -1.357308000 \\
1 & -2.376742000 & 1.407600000 & -1.677502000 \\
35 & -2.792928000 & -0.855996000 & -0.020015000 \\
1 & -0.667611000 & 1.915834000 & -2.133269000 \\
1 & 1.369702000 & 0.954224000 & 2.433422000 \\
1 & 1.342974000 & 1.208787000 & -1.750489000 \\
1 & 2.532024000 & 2.135049000 & 1.840840000 \\
7 & 2.817981000 & 0.167877000 & 1.105298000 \\
6 & 2.834204000 & 0.228207000 & -0.353294000 \\
1 & 3.663220000 & 0.879539000 & -0.678643000
\end{tabular}




$\begin{array}{lllr}1 & 2.406122000 & -0.717065000 & 1.379600000 \\ 6 & 3.055773000 & -1.096594000 & -1.040521000 \\ 1 & 2.841344000 & -0.964860000 & -2.110092000 \\ 1 & 2.352218000 & -1.835338000 & -0.649818000 \\ 8 & 4.394181000 & -1.474630000 & -0.834208000 \\ 1 & 4.488915000 & -2.408192000 & -1.014470000\end{array}$

$\begin{array}{lrrr}\text { TS3 } & & & \\ 6 & \text { AI } & & \\ 1 & -590395000 & 0.732104000 & -1.100632000 \\ 1 & -5.894533000 & 1.674166000 & -0.624649000 \\ 6 & -4.218920000 & -0.466743000 & 0.626594000 \\ 6 & -3.205767000 & 0.290967000 & 0.244232000 \\ 6 & -3.397215000 & 1.365153000 & -0.785186000 \\ 1 & -3.733691000 & 2.298883000 & -0.316526000 \\ 1 & -2.480842000 & 1.596168000 & -1.329463000 \\ 17 & -1.762965000 & -1.168917000 & -2.072320000 \\ 17 & -1.290899000 & 0.492639000 & 2.547222000 \\ 8 & -4.343636000 & 0.922286000 & -1.736831000 \\ 1 & -5.751516000 & -1.326947000 & -0.526247000 \\ 46 & -1.339051000 & -0.334444000 & 0.416263000 \\ 1 & -2.928388000 & -0.602333000 & -2.162497000 \\ 6 & 0.599855000 & -1.638237000 & 0.603890000 \\ 1 & 0.414993000 & -2.462990000 & -0.075194000 \\ 1 & 0.601665000 & -1.860538000 & 1.664649000 \\ 6 & -5.568868000 & -0.361900000 & -0.040099000 \\ 6 & 0.979876000 & -0.450582000 & 0.160708000 \\ 6 & 1.576933000 & 0.623599000 & -0.278144000 \\ 6 & 0.884534000 & 1.908802000 & -0.600926000 \\ 1 & 1.246203000 & 2.703669000 & 0.051227000 \\ 1 & -0.192352000 & 1.826376000 & -0.463843000 \\ 1 & 1.093809000 & 2.203270000 & -1.631463000 \\ 6 & 3.068384000 & 0.617311000 & -0.530013000 \\ 8 & 3.624556000 & 1.660474000 & 0.286217000 \\ 6 & 4.550408000 & 2.453933000 & -0.282518000 \\ 8 & 4.909222000 & 2.355208000 & -1.419531000 \\ 6 & 5.053170000 & 3.477169000 & 0.685490000 \\ 1 & 4.712308000 & 4.459168000 & 0.361210000 \\ 1 & 6.140049000 & 3.483954000 & 0.658211000 \\ 1 & 4.701622000 & 3.289946000 & 1.694707000 \\ 6 & 3.767826000 & -0.679501000 & -0.260302000 \\ 6 & 4.047374000 & -1.082737000 & 1.039117000 \\ 6 & 4.119416000 & -1.503202000 & -1.318133000 \\ 6 & 4.665457000 & -2.297309000 & 1.273463000 \\ 1 & 3.774693000 & -0.439333000 & 1.865702000 \\ 6 & 4.733165000 & -2.723050000 & -1.084542000 \\ 1 & 3.918762000 & -1.181415000 & -2.332615000 \\ 6 & 5.007380000 & -3.121222000 & 0.211955000 \\ 1 & 4.881460000 & -2.604182000 & 2.287710000 \\ 1 & 5.007011000 & -3.356391000 & -1.917146000 \\ 1 & 5.492307000 & -4.069969000 & 0.396741000 \\ 1 & 3.231118000 & 0.897166000 & -1.575201000 \\ 1 & -4.094379000 & -1.261928000 & 1.355188000 \\ 1 & -6.322115000 & 0.505931000 & -1.873100000 \\ 7 & -6.697535000 & -0.136502000 & 0.849267000 \\ 1 & -6.878325000 & -0.945379000 & 1.426495000 \\ 1 & -6.501894000 & 0.638299000 & 1.471219000\end{array}$




\begin{tabular}{|c|c|c|c|}
\hline \multicolumn{4}{|l|}{$\left|3_{6 \mathrm{~A}}\right|$} \\
\hline \multicolumn{4}{|c|}{ U } \\
\hline & 5.986149000 & -1.309947000 & 20tusto \\
\hline & 4.252172000 & 882451000 & 0.06856700 \\
\hline & 3.356748000 & -0.045246000 & -0.21179300 \\
\hline & 3.671604000 & -1.329646000 & -0.89869800 \\
\hline & 3.662136000 & -2.136266000 & 5263700 \\
\hline & 2.930166000 & -1.576364000 & 957800 \\
\hline 17 & .750944000 & 8000 & \\
\hline & 4 & & \\
\hline & 5.8 & & \\
\hline \multirow[t]{2}{*}{46} & 00 & 00 & \\
\hline & -0.47303 & 00 & \\
\hline & -0.328559000 & 000 & -0.6 \\
\hline 1 & -0.471027000 & 2.006312000 & $1.1926340 C$ \\
\hline & 5.694390000 & 0.726996000 & -0.38198000 \\
\hline & 646000 & 0000 & 100 \\
\hline & 000 & 000 & \\
\hline & 00 & & \\
\hline 1 & -1 & & \\
\hline I & & & \\
\hline 1 & -1.2 & & \\
\hline & -3.0 & -0 & \\
\hline & 524000 & -1. & 0. \\
\hline & -4.7 & -2.1 & $-0.0 \varsigma$ \\
\hline & -5.3 & -1 & 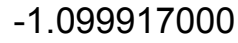 \\
\hline 6 & -5.2 & & \\
\hline 1 & -4.4 & & \\
\hline 1 & & & \\
\hline 1 & & & \\
\hline 6 & -3 & & \\
\hline 6 & -3 & & \\
\hline 6 & $-3 . \varepsilon$ & & \\
\hline 6 & 000 & 2.4 & 1. \\
\hline 1 & -3.6 & & 1. \\
\hline 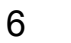 & -4.3 & & \\
\hline 1 & & & \\
\hline 0 & & & \\
\hline 1 & & & \\
\hline 1 & & & \\
\hline 1 & & & \\
\hline 1 & -3 & -0 & \\
\hline 1 & & & \\
\hline 1 & 6.8 & $-0 . \varepsilon$ & -1.2 \\
\hline 7 & 6.6 & & \\
\hline 1 & & & \\
\hline 1 & & & \\
\hline & & & \\
\hline 6 & & & \\
\hline 1 & & & -0.94775 \\
\hline 0 & -2 & & 0 \\
\hline 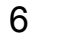 & & & \\
\hline & -2.801049000 & 51000 & 0.736722 \\
\hline & & -2.5652620 & -0 \\
\hline
\end{tabular}




\begin{tabular}{|c|c|c|c|}
\hline 1 & -2.408006000 & -2.333052000 & 1.617867000 \\
\hline 17 & 1.216426000 & -1.920645000 & -2.168237000 \\
\hline 8 & -4.103853000 & -1.403546000 & 1.042131000 \\
\hline 1 & -4.244829000 & 1.366141000 & 0.783325000 \\
\hline 46 & -0.155364000 & -1.193255000 & -0.477071000 \\
\hline 6 & -0.757782000 & -0.407230000 & 2.726816000 \\
\hline 1 & -1.822861000 & -0.423101000 & 2.907397000 \\
\hline 6 & -3.921780000 & 0.740457000 & -0.055915000 \\
\hline 1 & -0.114378000 & -0.221647000 & 3.580490000 \\
\hline 6 & -0.199809000 & -0.585702000 & 1.549875000 \\
\hline 6 & 1.126184000 & -0.659249000 & 1.059387000 \\
\hline 6 & 1.975183000 & -1.824570000 & 1.475801000 \\
\hline 1 & 2.704388000 & -2.083257000 & 0.710630000 \\
\hline 1 & 1.362688000 & -2.698065000 & 1.680670000 \\
\hline 1 & 2.519512000 & -1.551027000 & 2.3857 \\
\hline 6 & $1.8 \varepsilon$ & 0.626965000 & 0.779 \\
\hline 1 & 2.333811000 & 3364000 & 1.744791000 \\
\hline 1 & -1.799917000 & 1.388191000 & -0.102109000 \\
\hline 1 & -5.696151000 & -0.449763000 & 0.238299000 \\
\hline 7 & -4.325672000 & 1.414209000 & -1.277364000 \\
\hline 1 & -3.970772000 & 2.360234000 & -1.306396000 \\
\hline 1 & -3.950899000 & 0.929813000 & -2.084292000 \\
\hline 6 & 1.070723000 & 1.779380000 & 0.266881000 \\
\hline 6 & 0.518270000 & 2.683252000 & 1.162072000 \\
\hline 6 & ¡461000 & 3000 & -1.0953 \\
\hline 6 & -0.24 & 000 & 000 \\
\hline 1 & 0.694586000 & 2.558091000 & 2.223502000 \\
\hline 6 & 0.094568000 & 3.013702000 & -1.548456000 \\
\hline 1 & 1.290743000 & 1.251214000 & -1.796938000 \\
\hline 6 & -0.460976000 & 3.910523000 & -0.649272000 \\
\hline 1 & -0.669571000 & 4.444779000 & 1.415481000 \\
\hline 1 & -0.061922000 & 3.143870000 & -2.610337000 \\
\hline 1 & -1.048331000 & 4.744973000 & -1.007460000 \\
\hline 8 & 68000 & 1000 & -0.138190000 \\
\hline 6 & 4.18 & 000 & 0 \\
\hline 8 & 4.4 & 00 & 00 \\
\hline 6 & 5.092710000 & -0.180603000 & -0.7960 \\
\hline 1 & 4.688530000 & -1.059012000 & -1.299179000 \\
\hline 1 & 5.116932000 & 0.621125000 & -1.531779000 \\
\hline 1 & 6.091830000 & -0.374843000 & -0.422876000 \\
\hline \multicolumn{4}{|c|}{$\mid 4_{6 A \mid}$} \\
\hline 6 & -3.998738000 & -0.610313000 & -0.164674000 \\
\hline 1 & -3.535907000 & -1.422189000 & -0.749496000 \\
\hline 6 & -1.929930000 & 0.656195000 & 0.286572000 \\
\hline 6 & -1.652959000 & -0.291979000 & 1.241631000 \\
\hline 6 & -2.727689000 & -1.241693000 & 1.697972000 \\
\hline 1 & -2.4496 & -2.2 & 00 \\
\hline 1 & 0087000 & -1.196444000 & 2.787463000 \\
\hline 17 & 0.588367000 & -1.872836000 & -2.302768000 \\
\hline 8 & -3.995302000 & -0.921053000 & 1.204775000 \\
\hline 1 & -3.849019000 & 1.504615000 & 0.002215000 \\
\hline 46 & -0.609284000 & -0.860844000 & -0.633076000 \\
\hline 6 & -0.180252000 & -0.148416000 & 3.263465000 \\
\hline 1 & -1.011697000 & 0.053473000 & 3.925196000 \\
\hline & 034 & 0.686939000 & \\
\hline
\end{tabular}




$\begin{array}{rrrr}1 & 0.802522000 & -0.214801000 & 3.712750000 \\ 6 & -0.351998000 & -0.305640000 & 1.960870000 \\ 6 & 0.697135000 & -0.684951000 & 0.967813000 \\ 6 & 1.221338000 & -2.079808000 & 1.198121000 \\ 1 & 1.811222000 & -2.438133000 & 0.358283000 \\ 1 & 0.398234000 & -2.774954000 & 1.357669000 \\ 1 & 1.838391000 & -2.081578000 & 2.103586000 \\ 6 & 1.776593000 & 0.368145000 & 0.734889000 \\ 1 & 2.264494000 & 0.497237000 & 1.711905000 \\ 1 & -1.291950000 & 1.525166000 & 0.165228000 \\ 1 & -5.035969000 & -0.525078000 & -0.480188000 \\ 7 & -3.158498000 & 0.910290000 & -1.852271000 \\ 1 & -2.667068000 & 1.771086000 & -2.055049000 \\ 1 & -2.647615000 & 0.161941000 & -2.309381000 \\ 6 & 1.280814000 & 1.702530000 & 0.249743000 \\ 6 & 0.950932000 & 2.694542000 & 1.160578000 \\ 6 & 1.112314000 & 1.942027000 & -1.109410000 \\ 6 & 0.428769000 & 3.902664000 & 0.726741000 \\ 1 & 1.098032000 & 2.517852000 & 2.218532000 \\ 6 & 0.595760000 & 3.150321000 & -1.541991000 \\ 1 & 1.378168000 & 1.173832000 & -1.823244000 \\ 6 & 0.244712000 & 4.130054000 & -0.625658000 \\ 1 & 0.173785000 & 4.668046000 & 1.446385000 \\ 1 & 0.469433000 & 3.328789000 & -2.601175000 \\ 1 & -0.158261000 & 5.073467000 & -0.967392000 \\ 8 & 2.744907000 & -0.114342000 & -0.181279000 \\ 6 & 3.902982000 & -0.608937000 & 0.295854000 \\ 8 & 4.198650000 & -0.621791000 & 1.455293000 \\ 6 & 4.723158000 & -1.136100000 & -0.833328000 \\ 1 & 4.139969000 & -1.871862000 & -1.386975000 \\ 1 & 4.954564000 & -0.330154000 & -1.527344000 \\ 1 & 5.638352000 & -1.576921000 & -0.454593000\end{array}$

\section{TS4' ${ }_{6 \mathrm{AI}}$}

$-5.182632000$

$-5.216290000$ $-3.033506000$

$-2.480184000$

$-3.221390000$

$-3.316783000$

$-2.682862000$

0.435692000

$-4.488757000$

$-4.754794000$

$-0.730409000$

$-0.746007000$

$-1.804201000$

$-4.537329000$

$-0.091139000$

$-0.223467000$

0.961543000

1.593086000

2.089499000

0.851516000

2.351559000

1.836415000
$-0.158400000$

$-1.198648000$

0.527730000

$-0.192516000$

$-0.688678000$

$-1.783162000$

$-0.445115000$

$-2.586296000$

$-0.087309000$

1.757471000

$-0.990016000$

1.543386000

1.759160000

0.705298000

2.185648000

0.562608000

$-0.070951000$

$-1.038389000$

$-1.854549000$

$-1.446373000$

$-0.508409000$

0.565279000
0.473075000

0.113869000

$-0.546811000$

0.413464000

1.607934000

1.567085000

2.524127000

$-1.283262000$

1.692631000

$-0.381257000$

$-0.065546000$

1.927363000

1.964634000

$-0.595137000$

2.506146000

1.232071000

0.880653000

1.835348000

1.314615000

2.516971000

2.419150000

$-0.221232000$ 


\begin{tabular}{|c|c|c|c|}
\hline & 00 & 0.24730 & \\
\hline & -2.447747000 & 0.996891000 & -1 \\
\hline & -6.205745000 & 0.163703000 & \\
\hline & -5.142032000 & . 386552000 & \\
\hline & 6133000 & 1.027498000 & \\
\hline & -4.877177000 & -0.545318000 & -2.167 \\
\hline & 3.289932000 & 202582000 & -0.1063080 \\
\hline & 6313000 & -0.914551000 & -0.7847500 \\
\hline & 2490000 & 0.934488000 & 0.687020 \\
\hline & 30862000 & -1.297913000 & -0 \\
\hline & 384000 & 0390000 & \\
\hline & & 0 & \\
\hline & 3.80 & 1.8 & \\
\hline & 4000 & -0.5 & \\
\hline & 5.435288000 & -2.169412000 & -1.198 \\
\hline & 6.162604000 & 1.130301000 & 1.415038 \\
\hline & 6.984767000 & -0.862489000 & 0.2127830 \\
\hline & 7000 & 1.986940000 & -0.1385770 \\
\hline & 7000 & 2.574007000 & $-0 . \varsigma$ \\
\hline & 2000 & & \\
\hline & 0.7 & & \\
\hline & & & \\
\hline & & & \\
\hline & & & \\
\hline \multicolumn{4}{|c|}{14} \\
\hline & 4.13 & 00 & 0.58 \\
\hline & 3.80 & -1.3726 & 0.045 \\
\hline & 1.881276000 & 0.47390 & 0.951 \\
\hline & 62000 & 0.8 & $-0.2^{2}-x$ \\
\hline & 3.2 & & -1. \\
\hline & 3 & & \\
\hline & & & \\
\hline 7 & -0.0 & -3 . & \\
\hline & 0 & & -0 \\
\hline & 3.53 & 0.690 & 2.259 \\
\hline 46 & 502000 & -1.06 & -0.30 \\
\hline D & 0.73 & 2.4 & -1.914 \\
\hline 1 & 1.6 & & -2.0 \\
\hline 0 & 0 & $-0 .($ & \\
\hline 1 & -0 & & -2. \\
\hline 0 & & & \\
\hline & & & \\
\hline & & & \\
\hline 1 & -1 & -0.7 & -1 \\
\hline 1 & -0.37 & -0 & $-2 . \subseteq$ \\
\hline 1 & -1.706234000 & 0.891726 & -2.4988470 \\
\hline 6 & -1.364716000 & 0.793612 & 0.284 \\
\hline 1 & -0.833961000 & 0.76693 & 1.23 \\
\hline 1 & 1.068793000 & 0.77990 & 1.599 \\
\hline 1 & 5.08 & -0.68 & 1.051016 \\
\hline 7 & 2.84 & & 2.470 \\
\hline r & & & \\
\hline 1 & 2.3 & & \\
\hline 6 & -2.57 & & 0.38275 \\
\hline & -2.462 & -1.309956 & \\
\hline
\end{tabular}




$\begin{array}{lr}6 & -3.795543000 \\ 6 & -3.544456000 \\ 1 & -1.512513000 \\ 6 & -4.881418000 \\ 1 & -3.896333000 \\ 6 & -4.757542000 \\ 1 & -3.435763000 \\ 1 & -5.827935000 \\ 1 & -5.605109000 \\ 8 & -1.790004000 \\ 6 & -1.198724000 \\ 8 & -0.367955000 \\ 6 & -1.732023000 \\ 1 & -1.528680000 \\ 1 & -2.812179000 \\ 1 & -1.266714000\end{array}$

\section{0a-Pd}

\begin{tabular}{lc}
6 & -3.864589000 \\
1 & -3.620780000 \\
6 & -1.564071000 \\
6 & -1.493652000 \\
6 & -2.730385000 \\
1 & -2.600866000 \\
1 & -2.821683000 \\
17 & 1.218210000 \\
8 & -3.909357000 \\
1 & -3.240775000 \\
46 & -0.219655000 \\
6 & -0.446020000 \\
1 & -1.384320000 \\
6 & -2.847387000 \\
1 & 0.405229000 \\
6 & -0.333189000 \\
6 & 0.929305000 \\
6 & 1.607476000 \\
1 & 2.331996000 \\
1 & 0.871020000 \\
1 & 2.115913000 \\
6 & 1.467883000 \\
1 & 0.983732000 \\
8 & -0.844442000 \\
6 & -1.572400000 \\
8 & -1.657926000 \\
6 & -2.534312000 \\
1 & -2.236364000 \\
1 & -3.522800000 \\
1 & -2.599016000 \\
6 & 2.768134000 \\
6 & 2.842313000 \\
6 & 3.930124000 \\
6 & 4.040166000 \\
1 & 1.935435000 \\
6 & 5.134774000 \\
1 & 3.904535000 \\
6 & 5.191532000 \\
& \\
\hline
\end{tabular}

0.269574000
-2.167482000
-1.604196000
-0.583631000
1.222587000
-1.805618000
-3.121143000
-0.292511000
-2.473807000
2.147421000
3.109689000
2.901119000
4.451637000
4.640789000
4.473511000
5.217551000

$-0.193284000$

0.518609000

$-0.784483000$

$-1.678282000$

$-1.894522000$

$-1.369854000$

$-2.956703000$

1.104890000

$-1.512767000$

$-0.695521000$

0.077774000

$-3.894460000$

$-4.400004000$

$-0.096187000$

$-4.515859000$

$-2.576651000$

$-1.842275000$

$-2.225330000$

$-1.479943000$

$-2.347944000$

$-3.184757000$

$-0.986641000$

$-0.953843000$

2.795199000

2.518376000

1.346135000

3.514105000

4.521982000

3.316565000

3.406597000

$-0.307746000$

0.936883000

$-0.873203000$

1.614548000

1.390011000

$-0.198965000$

$-1.854303000$

1.047081000
$-0.172445000$

1.112519000

1.463387000

$-0.084073000$

$-0.672300000$

0.553756000

1.609474000

$-0.518430000$

0.616602000

0.066086000

0.799728000

1.635735000

0.419672000

$-0.633243000$

0.549592000

1.029727000

0.616429000

$-0.177789000$

1.358743000

0.322153000

$-0.520794000$

$-1.479848000$

$-0.748051000$

$-1.860006000$

0.115078000

2.568842000

$-0.391659000$

$-0.032109000$

0.147030000

1.738082000

$-0.270978000$

0.065798000

$-0.244720000$

$-1.519809000$

$-1.831392000$

$-2.312604000$

$-1.391600000$

0.698393000

1.665698000

0.406177000

$-0.534233000$

$-1.072381000$

$-1.123012000$

$-0.851787000$

$-0.704704000$

$-2.202131000$

0.710321000

1.335012000

0.197634000

1.411285000

1.718931000

0.290963000

$-0.254302000$

0.887918000 


$\begin{array}{rrrr}1 & 4.076962000 & 2.590067000 & 1.874896000 \\ 1 & 6.033040000 & -0.651612000 & -0.105292000 \\ 1 & 6.132716000 & 1.575430000 & 0.949468000 \\ 1 & -0.802939000 & -0.759476000 & 2.127560000 \\ 1 & -4.854844000 & 0.039465000 & 1.000877000 \\ 7 & -2.719704000 & 1.262927000 & 2.209866000 \\ 1 & -2.517735000 & 1.308844000 & 3.195513000 \\ 1 & -2.016372000 & 1.798708000 & 1.709683000\end{array}$

\section{$\mathrm{TS}_{5 \mathrm{~A}}$}

$\begin{array}{lrrr}6 & -4.867391000 & -0.821305000 & -0.478731000 \\ 1 & -4.207615000 & -0.730192000 & -1.351935000 \\ 6 & -4.130750000 & -0.303465000 & 0.742969000 \\ 6 & -2.840519000 & -1.038395000 & 0.948516000 \\ 6 & -1.830747000 & -0.272179000 & 0.547299000 \\ 6 & -2.295105000 & 1.093252000 & 0.145806000 \\ 1 & -2.178347000 & 1.234436000 & -0.938178000 \\ 1 & -1.716782000 & 1.884466000 & 0.629706000 \\ 8 & -6.032745000 & -0.043972000 & -0.628844000 \\ 1 & -6.495096000 & -0.309205000 & -1.422290000 \\ 1 & -2.775310000 & -2.043307000 & 1.338129000 \\ 1 & -5.105606000 & -1.883414000 & -0.344116000 \\ 1 & -4.804000000 & -0.398914000 & 1.602735000 \\ 7 & -3.682420000 & 1.080596000 & 0.604811000 \\ 1 & -4.311599000 & 1.622119000 & 0.030503000 \\ 46 & -0.229859000 & -1.195095000 & -0.292417000 \\ 17 & 0.973570000 & -2.272454000 & -1.921198000 \\ 6 & -0.102041000 & -0.311440000 & 1.635745000 \\ 6 & 1.179721000 & -0.583820000 & 1.082471000 \\ 6 & -0.533672000 & 0.080348000 & 2.815931000 \\ 6 & 1.929939000 & -1.763738000 & 1.628163000 \\ 6 & 2.045583000 & 0.574920000 & 0.626854000 \\ 1 & -1.575418000 & 0.212852000 & 3.064612000 \\ 1 & 0.202692000 & 0.310167000 & 3.579898000 \\ 1 & 2.614756000 & -2.178943000 & 0.891477000 \\ 1 & 1.245926000 & -2.545253000 & 1.946905000 \\ 1 & 2.514721000 & -1.434135000 & 2.493754000 \\ 1 & 2.528892000 & 0.936905000 & 1.545714000 \\ 6 & 1.332966000 & 1.719599000 & -0.032606000 \\ 8 & 3.060370000 & 0.092293000 & -0.243761000 \\ 6 & 1.002005000 & 2.840230000 & 0.713482000 \\ 6 & 0.998552000 & 1.672234000 & -1.380858000 \\ 6 & 4.304882000 & -0.070357000 & 0.244965000 \\ 6 & 0.333235000 & 3.902259000 & 0.128098000 \\ 1 & 1.273340000 & 2.881899000 & 1.761350000 \\ 6 & 0.334650000 & 2.735632000 & -1.965504000 \\ 1 & 1.261036000 & 0.800024000 & -1.966246000 \\ 8 & 4.624739000 & 0.197130000 & 1.366634000 \\ 6 & 5.188521000 & -0.646543000 & -0.809975000 \\ 6 & -0.001261000 & 3.850284000 & -1.213278000 \\ 1 & 0.081731000 & 4.772300000 & 0.718463000 \\ 1 & 0.080585000 & 2.694784000 & -3.015704000 \\ 1 & 4.777450000 & -1.601080000 & -1.138457000 \\ 1 & 5.203298000 & 0.006743000 & -1.679999000 \\ 1 & 6.191717000 & -0.779698000 & -0.421098000 \\ 1 & -0.516918000 & 4.680641000 & -1.675659000\end{array}$




\begin{tabular}{|c|c|c|c|}
\hline \multicolumn{4}{|c|}{$14_{5 \mathrm{AI}}$} \\
\hline 6 & -3.843835000 & -1.119143000 & -0.522718000 \\
\hline 1 & -3.001116000 & -1.048973000 & -1.232569000 \\
\hline 6 & -3.548130000 & -0.249884000 & 0.681353000 \\
\hline 6 & -2.248477000 & -0.616574000 & 1.338067000 \\
\hline 6 & -1.397881000 & 0.457095000 & 1.333965000 \\
\hline 6 & -2.076190000 & 1.650989000 & 0.728378000 \\
\hline 1 & -1.508568000 & 2.067806000 & -0.107595000 \\
\hline 1 & -2.136834000 & 2.440570000 & 1.491081000 \\
\hline 8 & -5.032444000 & -0.654122000 & -1.108546000 \\
\hline 1 & -5.214456000 & -1.155008000 & -1.902659000 \\
\hline 1 & -2.141322000 & -1.492608000 & 1.964182000 \\
\hline 1 & -3.924580000 & -2.165695000 & -0.205427000 \\
\hline 1 & -4.374008000 & -0.386888000 & 1.393271000 \\
\hline 7 & -3.385679000 & 7461000 & 6000 \\
\hline 1 & -3.649223000 & 1.358831000 & -0.613056000 \\
\hline 46 & -0.655593000 & -1.011582000 & -0.154686000 \\
\hline 17 & 0.453794000 & -2.246412000 & -1.736312000 \\
\hline 6 & -0.082442000 & 0.471260000 & 2.004916000 \\
\hline 6 & 0.817344000 & -0.390917000 & 1.183562000 \\
\hline 6 & 0.224114000 & 1.122994000 & 3.113711000 \\
\hline 6 & 1.312152000 & -1.625899000 & 1.883382000 \\
\hline 6 & 1.902138000 & 0.420123000 & 0.477491000 \\
\hline 1 & -0.508228000 & 1.729444000 & 9000 \\
\hline 1 & 1.216533000 & 1.069398000 & 3.542809000 \\
\hline 1 & 1.818739000 & -2.306921000 & 1.203312000 \\
\hline 1 & 0.489038000 & -2.158386000 & 2.356902000 \\
\hline 1 & 2.016632000 & -1.328863000 & 2.668639000 \\
\hline 1 & 2.554470000 & 0.766190000 & 1.291830000 \\
\hline 6 & 1.420764000 & 1.615938000 & -0.300075000 \\
\hline 8 & 2.669661000 & -0.404957000 & -0.380898000 \\
\hline 6 & 1.453574000 & 2.875397000 & 0.281749000 \\
\hline 6 & 000 & 34000 & 2000 \\
\hline 6 & 053000 & -0.8 & 0000 \\
\hline 6 & 0.999713000 & 3.985850000 & -0.409872000 \\
\hline 1 & 1.840889000 & 2.987562000 & 1.286610000 \\
\hline 6 & 0.496312000 & 2.593568000 & -2.291911000 \\
\hline 1 & 0.938751000 & 0.506801000 & -2.070251000 \\
\hline 8 & 4.334078000 & -0.608487000 & 1.116102000 \\
\hline 6 & 4.422363000 & -1.803087000 & -0.967921000 \\
\hline 6 & 0.516276000 & 3.845662000 & -1.698610000 \\
\hline 1 & 1.033744000 & 4.961016000 & 0.055885000 \\
\hline 1 & 0.133963000 & 00 & 8000 \\
\hline 1 & 3.700760000 & -2.593501000 & -1.174787000 \\
\hline 1 & 4.574969000 & -1.266596000 & -1.902505000 \\
\hline 1 & 5.359802000 & -2.217268000 & -0.614188000 \\
\hline 1 & 0.166956000 & 4.711271000 & -2.244402000 \\
\hline \multicolumn{4}{|l|}{ TS } \\
\hline 6 & $-4.95 \varsigma$ & 000 & 2000 \\
\hline 1 & -4.212112000 & 1.124086000 & 1.530562000 \\
\hline 6 & -4.241855000 & -0.580087000 & 0.238581000 \\
\hline 6 & -3.235530000 & 0.000047000 & -0.711808000 \\
\hline 6 & -2.017482000 & -0.180955000 & -0.218093000 \\
\hline 6 & -2.029581000 & -0.995327000 & 1.034859000 \\
\hline
\end{tabular}




\begin{tabular}{lrrr}
1 & -1.717287000 & -0.377859000 & 1.891262000 \\
1 & -1.339406000 & -1.842245000 & 0.985935000 \\
8 & -5.844488000 & -0.108711000 & 1.904945000 \\
1 & -6.269288000 & 0.553222000 & 2.448166000 \\
1 & -3.502197000 & 0.515861000 & -1.622947000 \\
1 & -5.493670000 & 1.168095000 & 0.302023000 \\
1 & -5.002046000 & -1.177953000 & -0.278333000 \\
7 & -3.419696000 & -1.436624000 & 1.089162000 \\
1 & -3.794985000 & -1.519092000 & 2.022026000 \\
46 & -0.608100000 & 1.164714000 & -0.700142000 \\
17 & 0.259902000 & 3.278096000 & -0.520091000 \\
6 & -0.386619000 & -0.721115000 & -1.516100000 \\
6 & 0.882588000 & -0.221687000 & -1.157065000 \\
6 & -0.915099000 & -1.688478000 & -2.230982000 \\
6 & 1.810892000 & 0.293043000 & -2.209313000 \\
6 & 1.520921000 & -0.807528000 & 0.096206000 \\
1 & -1.976815000 & -1.820279000 & -2.366585000 \\
1 & -0.249432000 & -2.407410000 & -2.697214000 \\
1 & 2.446080000 & 1.086820000 & -1.820680000 \\
1 & 1.263328000 & 0.663148000 & -3.071451000 \\
1 & 2.456136000 & -0.531961000 & -2.530384000 \\
1 & 0.754053000 & -0.981091000 & 0.850948000 \\
6 & 2.628097000 & 0.016979000 & 0.685535000 \\
8 & 2.020586000 & -2.082327000 & -0.335321000 \\
6 & 2.301015000 & 1.058266000 & 1.541852000 \\
6 & 3.960232000 & -0.215175000 & 0.374850000 \\
6 & 1.565131000 & -3.188287000 & 0.292862000 \\
6 & 3.289463000 & 1.866180000 & 2.071746000 \\
1 & 1.261818000 & 1.249601000 & 1.782266000 \\
6 & 4.950528000 & 0.587127000 & 0.914448000 \\
1 & 4.220541000 & -1.029882000 & -0.286999000 \\
8 & 0.780961000 & -3.174149000 & 1.193218000 \\
6 & 2.185601000 & -4.408117000 & -0.306163000 \\
6 & 4.617435000 & 1.630468000 & 1.760329000 \\
1 & 3.019799000 & 2.681845000 & 2.727474000 \\
1 & 5.986685000 & 0.397200000 & 0.670361000 \\
1 & -2.837808000 & 0.624171000 & 1.831323000 \\
1 & -4.491923000 & -1.325766000 & 0.544676000 \\
1 & -4.645305000 & 1.047022000 & 0.046114000 \\
1 & -3.175088000 & 1.086793000 & -1.474561000 \\
1 & 1.812315000 & -5.292876000 & -0.213958000 \\
1 & 1.953924000 & -4.456955000 & -1.368778000 \\
1 & 5.391990000 & 2.259176000 & 2.177055000 \\
6 & -1.835730000 & 2.741775000 & -1.344343000 \\
6 & -4.106012000 & -0.949675000 & -0.410765000 \\
6 & -3.197236000 & -1.526734000 & -0.654559000 \\
6 & -3.735832000 & 0.510280000 & -0.259576000 \\
6 & -2.662920000 & 0.715676000 & 0.767275000 \\
\hline & -1.856137000 & 1.322796000 & 0.208368000 \\
\hline & -1.100256000 & 1.649324000 & -1.231401000 \\
\hline & & & \\
1 & -5.266511000 & -1.971079000 & -1.591068000 \\
\hline
\end{tabular}




$\begin{array}{lrrr}1 & -3.228928000 & 0.463311000 & -2.262701000 \\ 46 & -0.996687000 & -0.796580000 & 0.558386000 \\ 17 & 0.019652000 & -2.822994000 & 0.787756000 \\ 6 & -0.394392000 & 1.723516000 & 1.009123000 \\ 6 & 0.531408000 & 0.563400000 & 0.998291000 \\ 6 & -0.285947000 & 2.846040000 & 1.702418000 \\ 6 & 1.163072000 & 0.200544000 & 2.307574000 \\ 6 & 1.459781000 & 0.614615000 & -0.222727000 \\ 1 & -1.053816000 & 3.606305000 & 1.653977000 \\ 1 & 0.572739000 & 3.039852000 & 2.331379000 \\ 1 & 1.650693000 & -0.769395000 & 2.262236000 \\ 1 & 0.417691000 & 0.182334000 & 3.100006000 \\ 1 & 1.913235000 & 0.955877000 & 2.568659000 \\ 1 & 0.859516000 & 0.806828000 & -1.111515000 \\ 6 & 2.350997000 & -0.561982000 & -0.483757000 \\ 8 & 2.288966000 & 1.777946000 & -0.000193000 \\ 6 & 2.052050000 & -1.415096000 & -1.534146000 \\ 6 & 3.478493000 & -0.811702000 & 0.287432000 \\ 6 & 2.072784000 & 2.860714000 & -0.768936000 \\ 6 & 2.845684000 & -2.515958000 & -1.797943000 \\ 1 & 1.176972000 & -1.223396000 & -2.143216000 \\ 6 & 4.271965000 & -1.913691000 & 0.028205000 \\ 1 & 3.743511000 & -0.133766000 & 1.087234000 \\ 8 & 1.263989000 & 2.915140000 & -1.649046000 \\ 6 & 2.973260000 & 3.977569000 & -0.350888000 \\ 6 & 3.953827000 & -2.771614000 & -1.011310000 \\ 1 & 2.593427000 & -3.177751000 & -2.614400000 \\ 1 & 5.144447000 & -2.102439000 & 0.638304000 \\ 1 & 4.001161000 & 3.631344000 & -0.273406000 \\ 1 & 2.898709000 & 4.794116000 & -1.060443000 \\ 1 & 2.669888000 & 4.324605000 & 0.636869000 \\ 1 & 4.573489000 & -3.634881000 & -1.210381000\end{array}$

\section{1-Pd}

$\begin{array}{lccc}6 & 3.104475000 & 1.794054000 & -0.654422000 \\ 6 & 2.054229000 & 1.909315000 & 1.422377000 \\ 1 & 1.880609000 & 1.248233000 & 2.271291000 \\ 1 & 2.025904000 & 2.938461000 & 1.799536000 \\ 17 & -0.631868000 & -2.291648000 & -0.272657000 \\ 46 & 0.586964000 & -0.354497000 & -0.281078000 \\ 6 & -0.666022000 & 3.205001000 & 1.429739000 \\ 1 & 0.119282000 & 3.749358000 & 1.931544000 \\ 1 & -1.676958000 & 3.553688000 & 1.581047000 \\ 6 & -0.417034000 & 2.170038000 & 0.624445000 \\ 6 & -1.524698000 & 1.394112000 & 0.018319000 \\ 6 & -1.384423000 & 0.898488000 & -1.390010000 \\ 1 & -2.239101000 & 0.296562000 & -1.683088000 \\ 1 & -1.235704000 & 1.714269000 & -2.100882000 \\ 1 & -0.527972000 & 0.209947000 & -1.656065000 \\ 6 & -2.602860000 & 1.109391000 & 0.761338000 \\ 1 & -2.559651000 & 1.382497000 & 1.810618000 \\ 8 & 2.719968000 & -2.377447000 & -0.610272000 \\ 6 & 2.627501000 & -2.071458000 & 0.561280000 \\ 8 & 1.861076000 & -1.133607000 & 1.035084000 \\ 6 & 3.483746000 & -2.707166000 & 1.623699000 \\ 1 & 4.468278000 & -2.240855000 & 1.570731000\end{array}$




$\begin{array}{rrrr}1 & 3.077096000 & -2.570363000 & 2.620399000 \\ 1 & 3.599908000 & -3.763040000 & 1.395992000 \\ 6 & -3.821788000 & 0.411332000 & 0.349763000 \\ 6 & -4.311572000 & -0.623970000 & 1.143039000 \\ 6 & -4.540048000 & 0.775831000 & -0.786755000 \\ 6 & -5.465441000 & -1.296547000 & 0.792200000 \\ 1 & -3.762178000 & -0.912190000 & 2.029954000 \\ 6 & -5.701637000 & 0.109520000 & -1.132021000 \\ 1 & -4.198470000 & 1.608994000 & -1.387975000 \\ 6 & -6.163920000 & -0.932356000 & -0.347329000 \\ 1 & -5.822268000 & -2.108884000 & 1.409856000 \\ 1 & -6.251552000 & 0.409953000 & -2.013170000 \\ 1 & -7.070122000 & -1.455613000 & -0.618757000 \\ 6 & 0.982626000 & 1.771967000 & 0.360292000 \\ 6 & 1.622384000 & 1.587410000 & -0.847600000 \\ 1 & 1.173520000 & 1.754242000 & -1.818290000 \\ 1 & 3.260472000 & 2.848105000 & -0.942739000 \\ 7 & 3.320546000 & 1.638102000 & 0.771170000 \\ 1 & 3.609901000 & 0.680562000 & 0.933368000 \\ 6 & 4.102727000 & 0.998093000 & -1.487947000 \\ 1 & 5.039101000 & 1.557948000 & -1.499967000 \\ 1 & 3.740282000 & 0.953255000 & -2.521880000 \\ 8 & 4.400884000 & -0.258904000 & -0.974598000 \\ 1 & 3.722347000 & -0.915657000 & -1.189087000\end{array}$



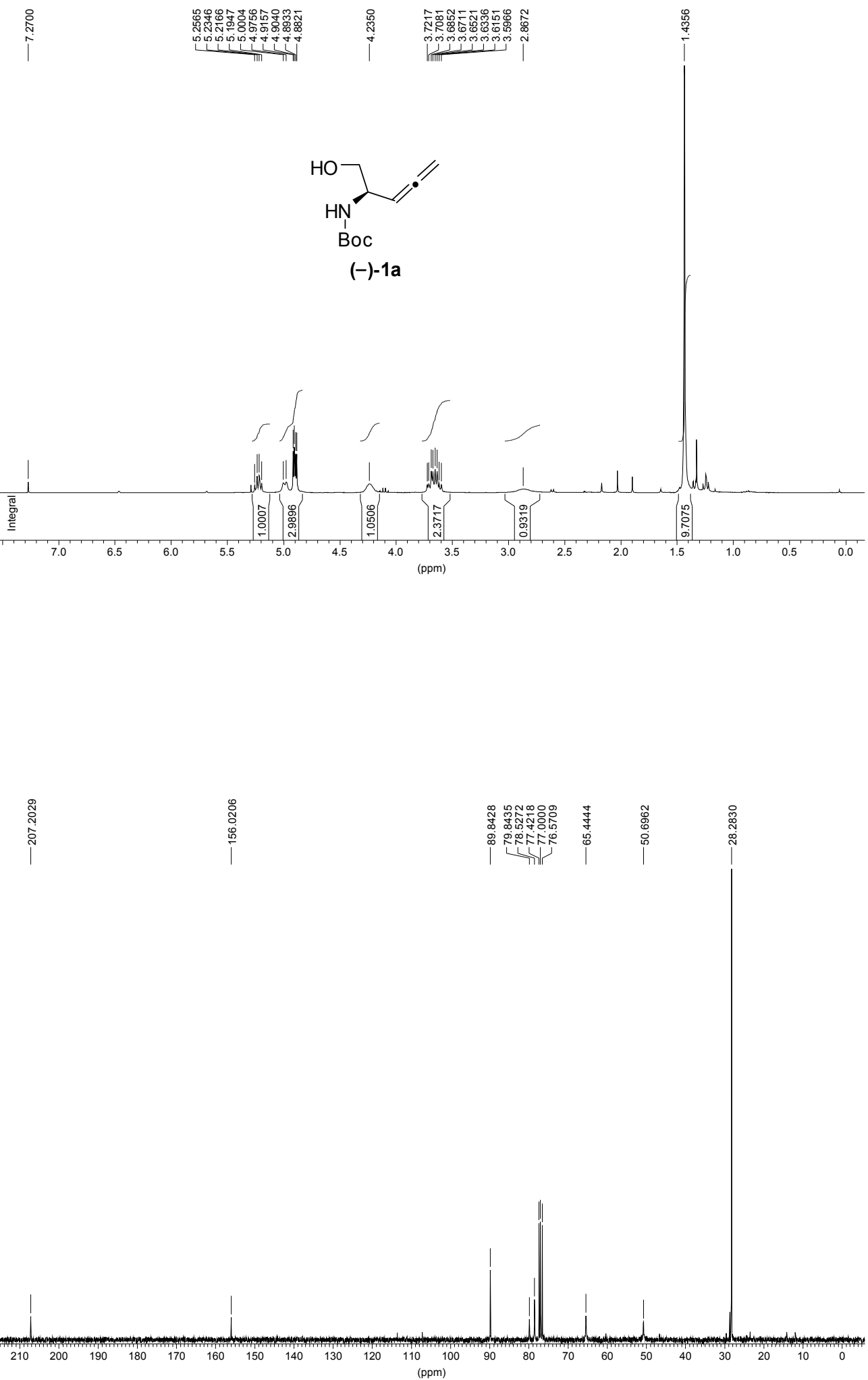


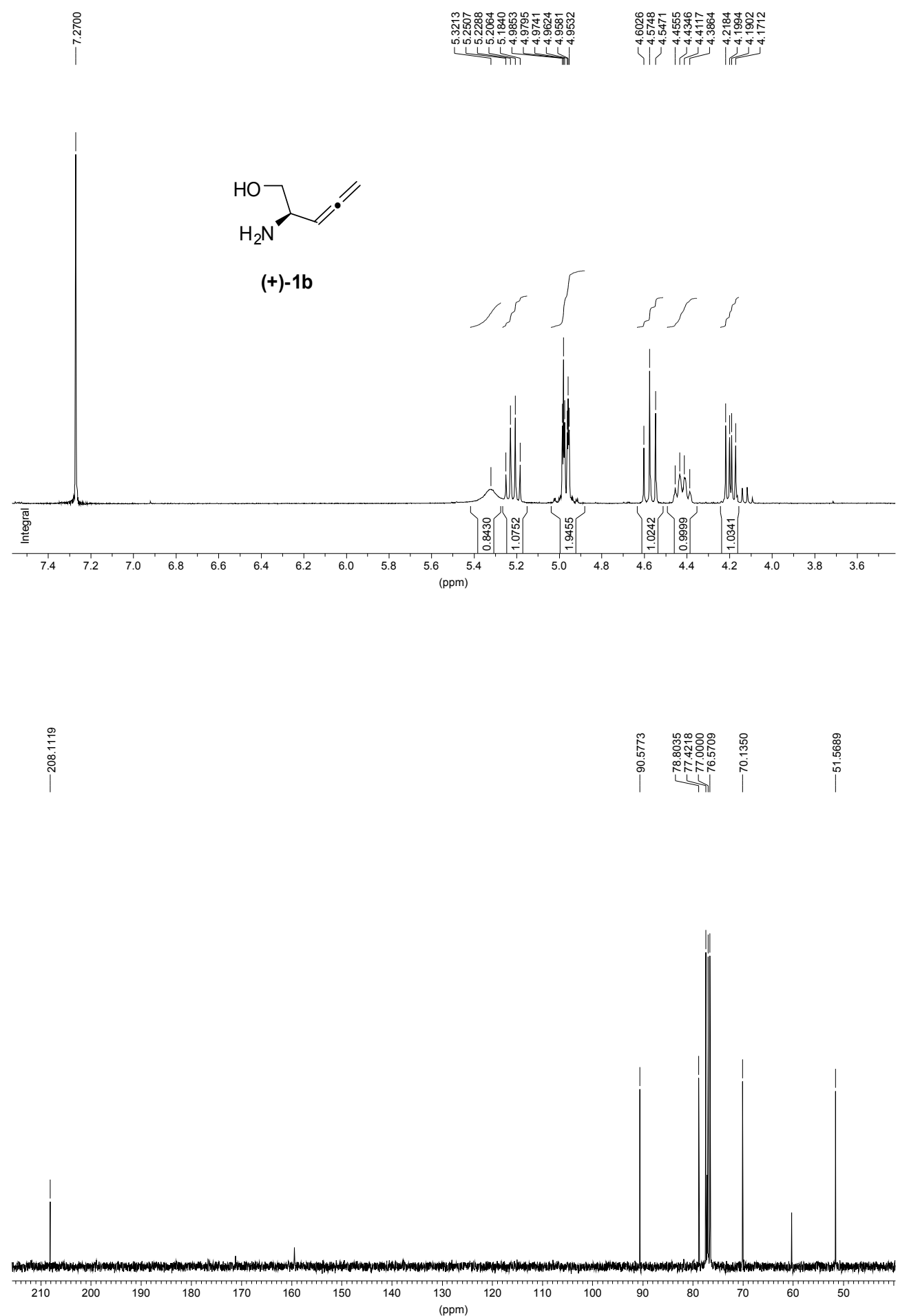



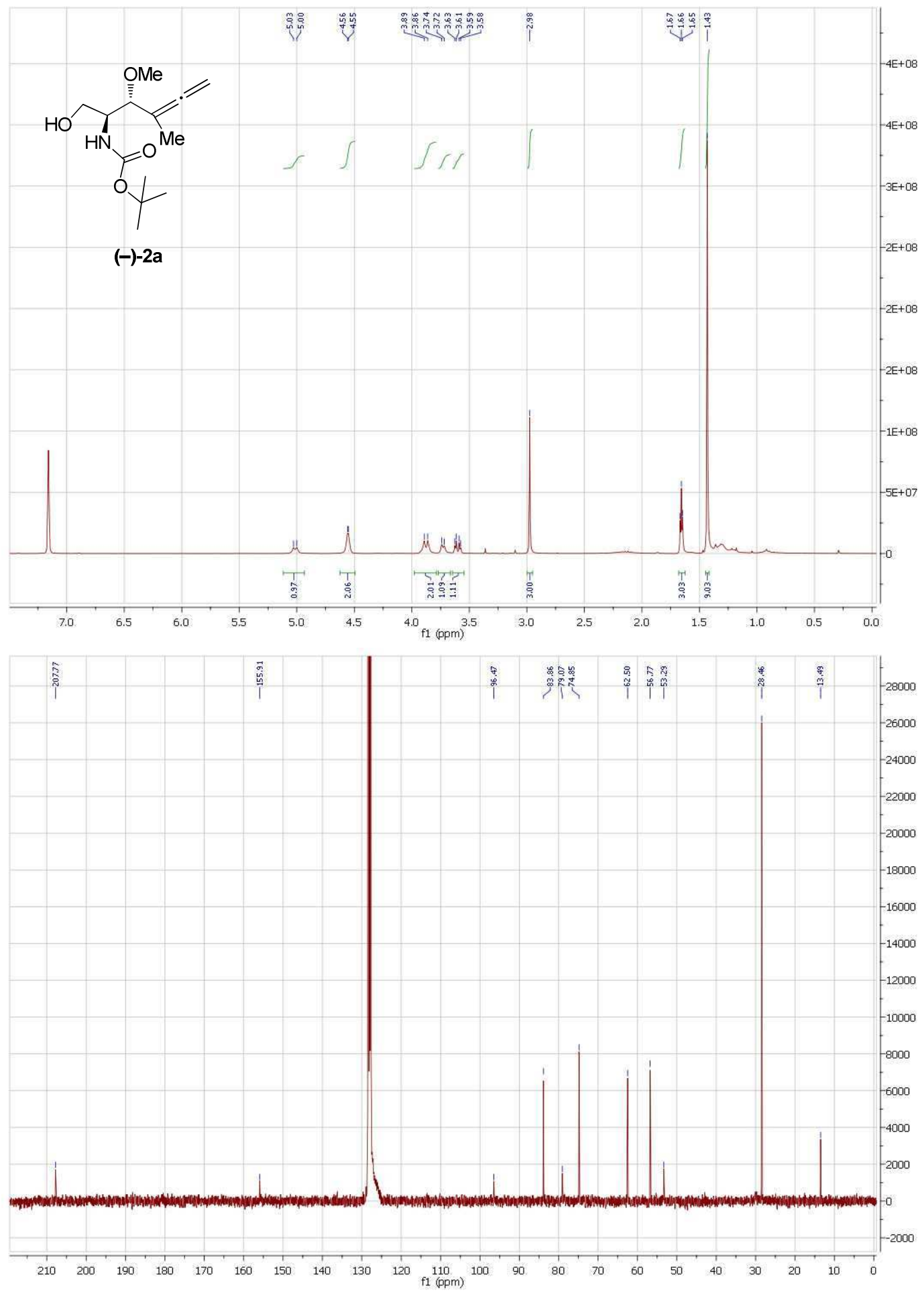

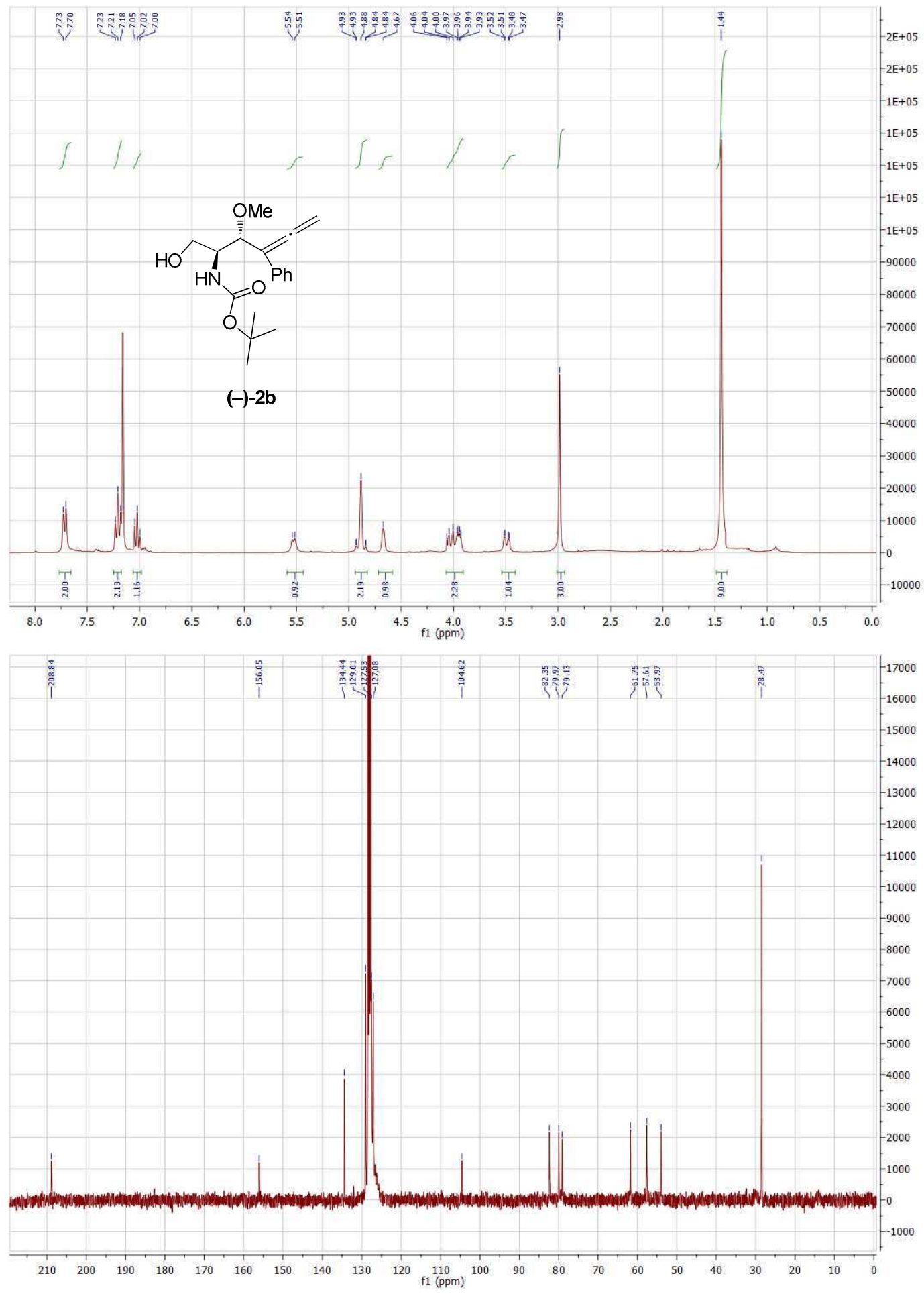

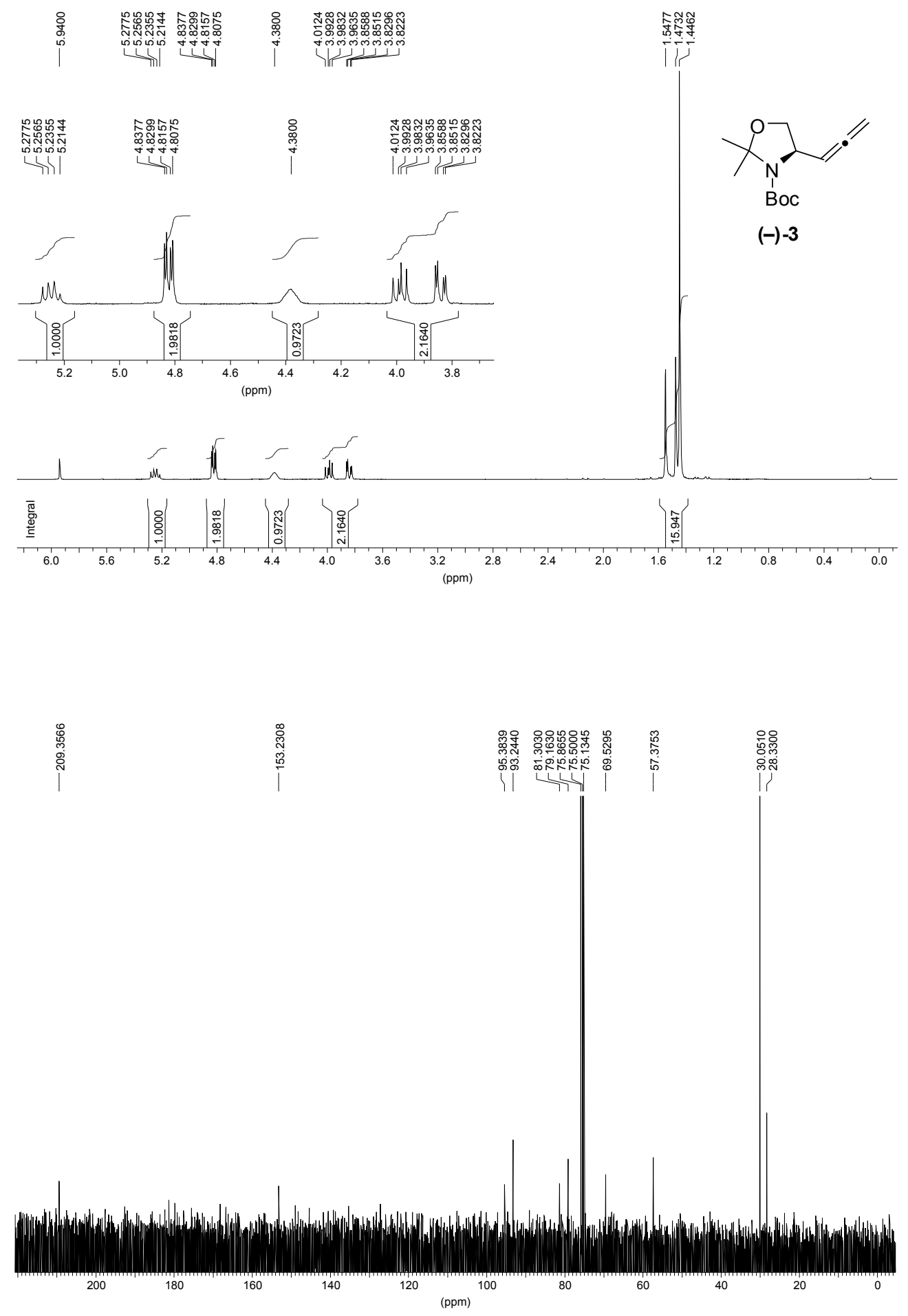


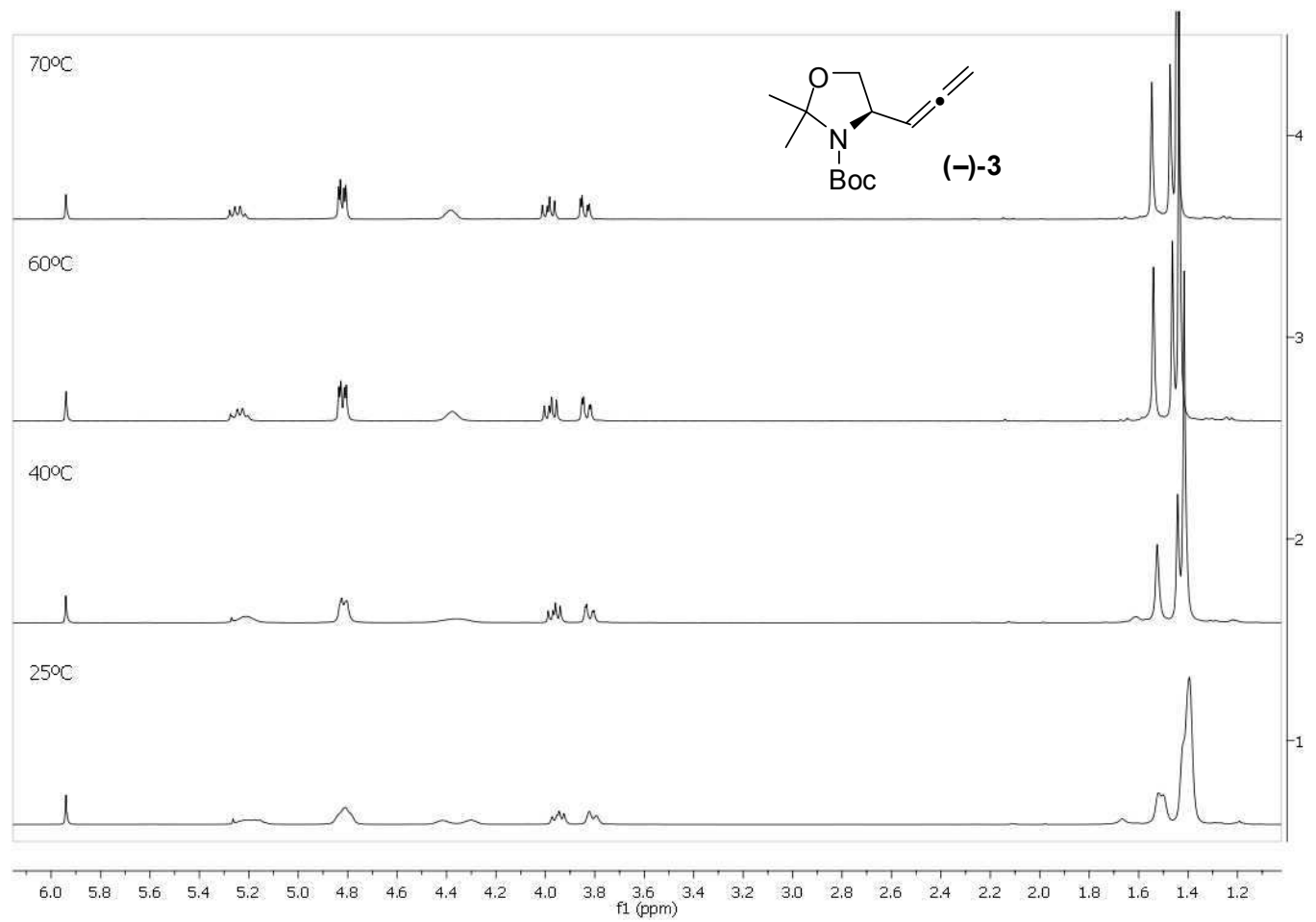



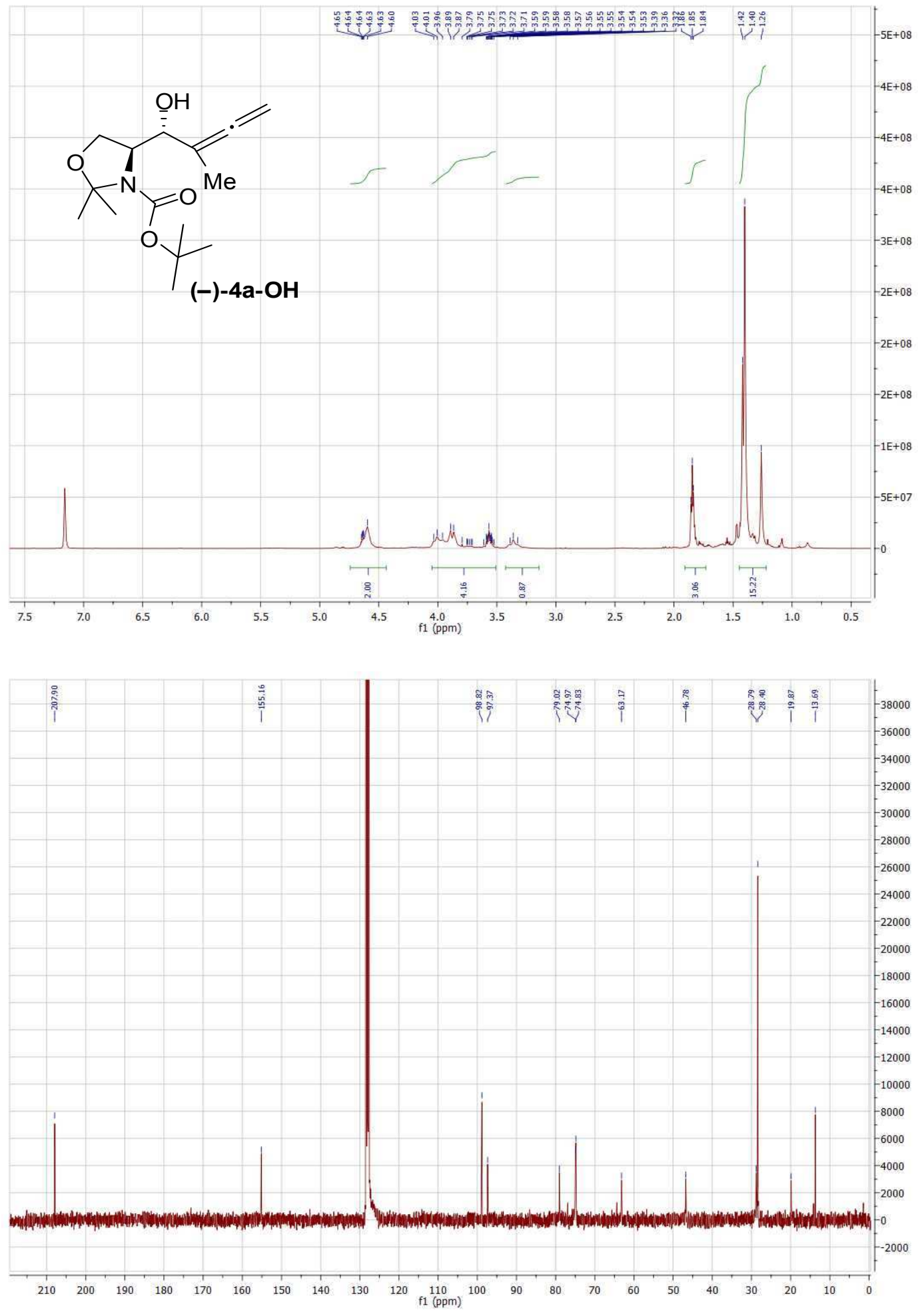

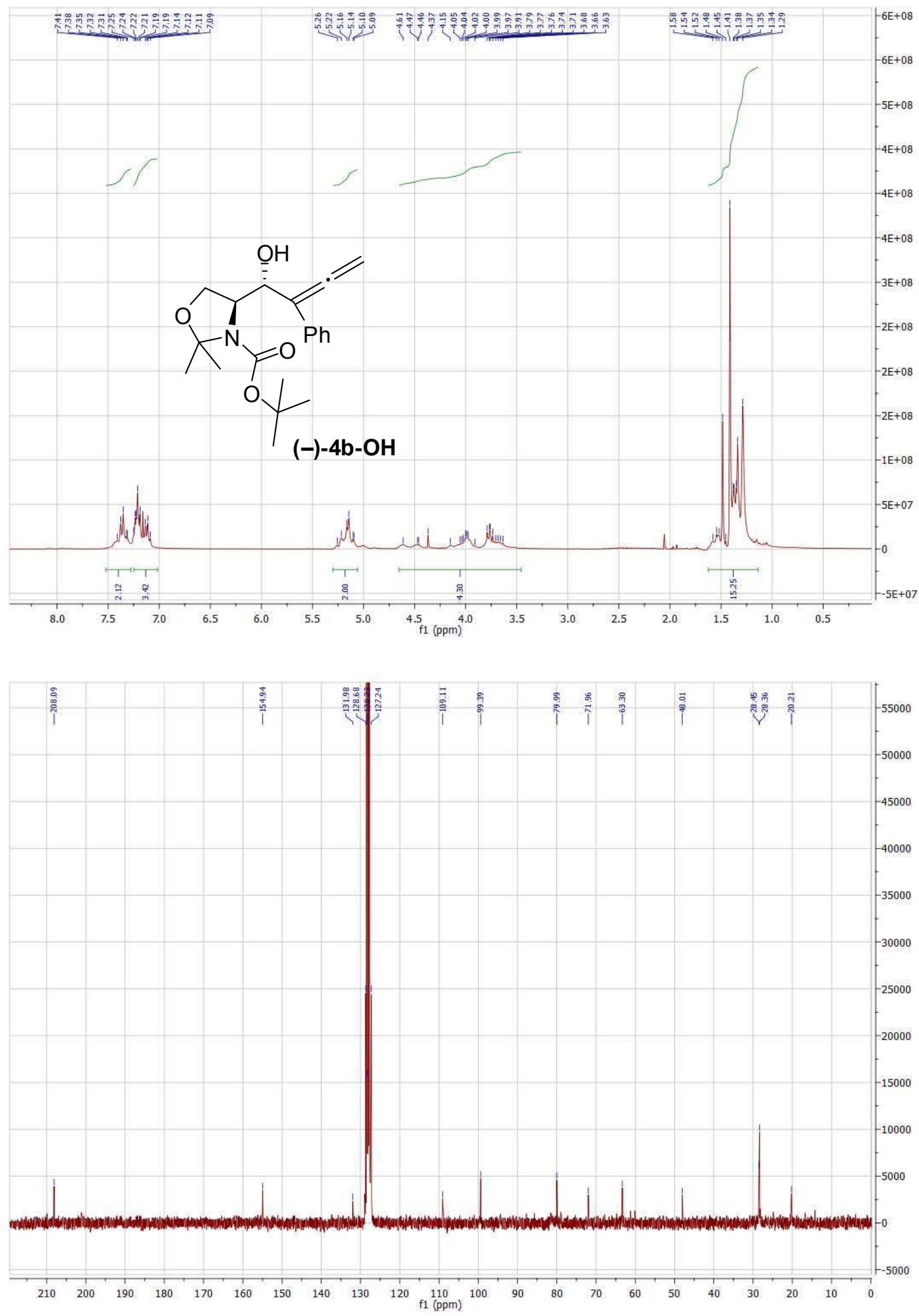

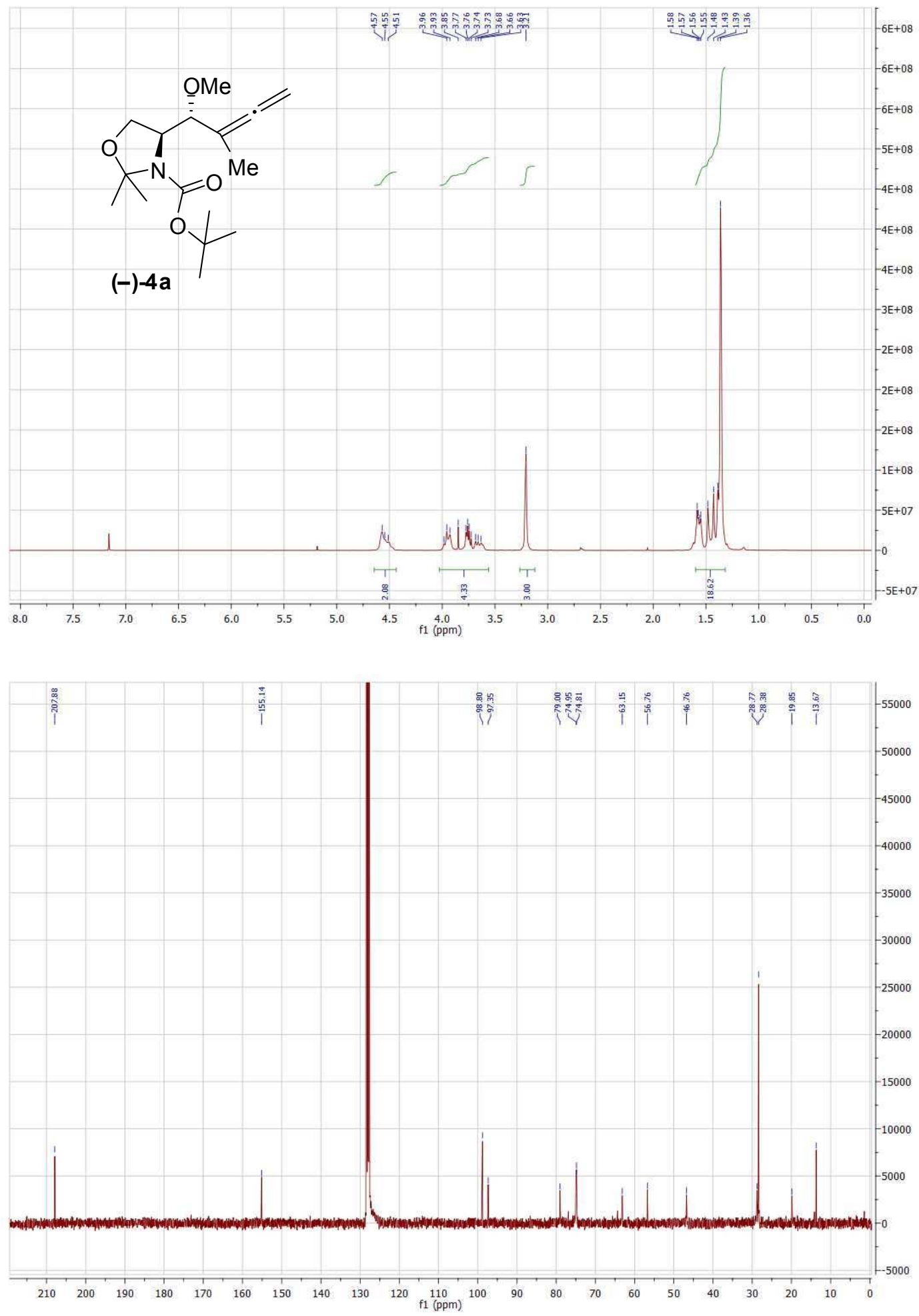

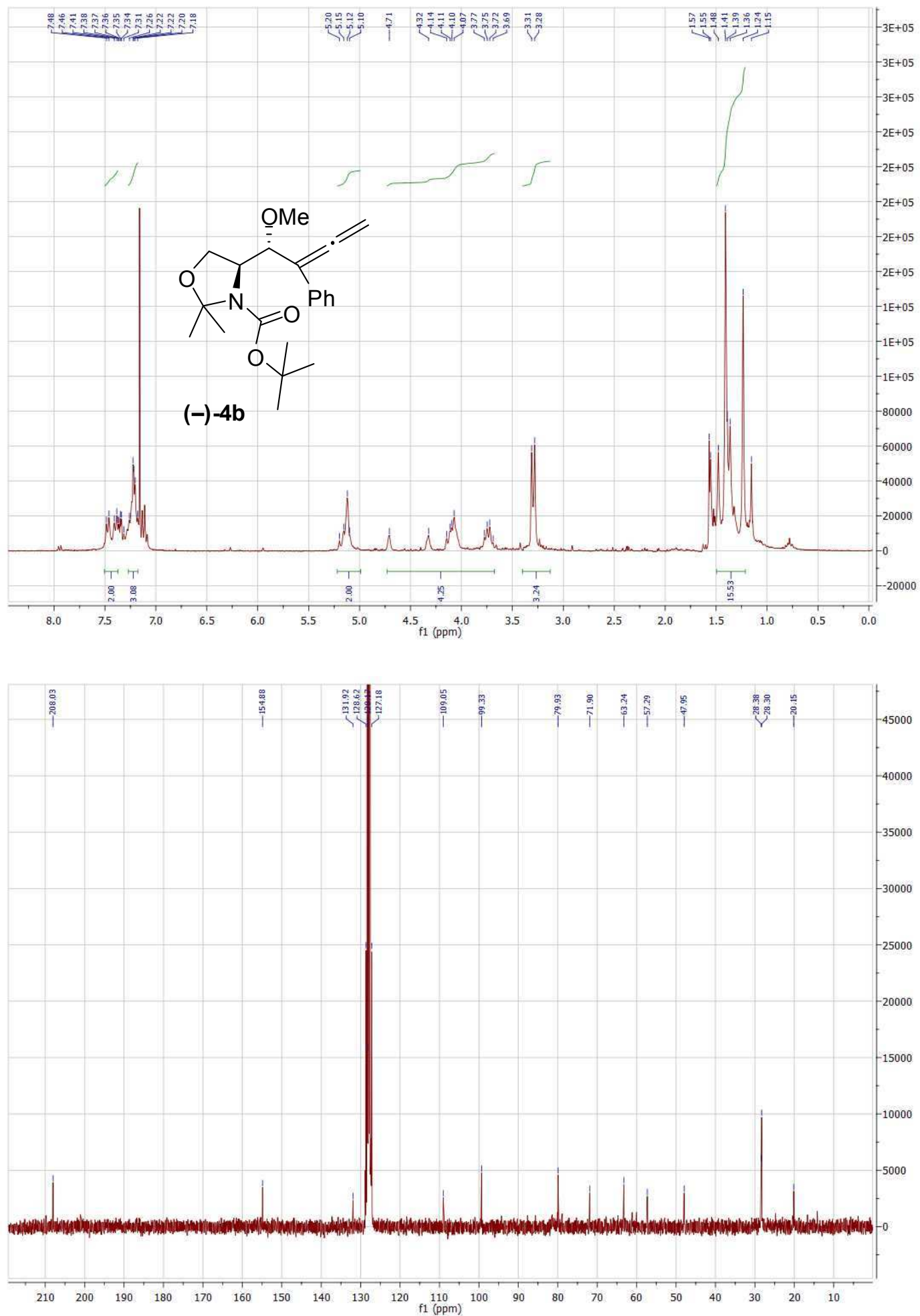

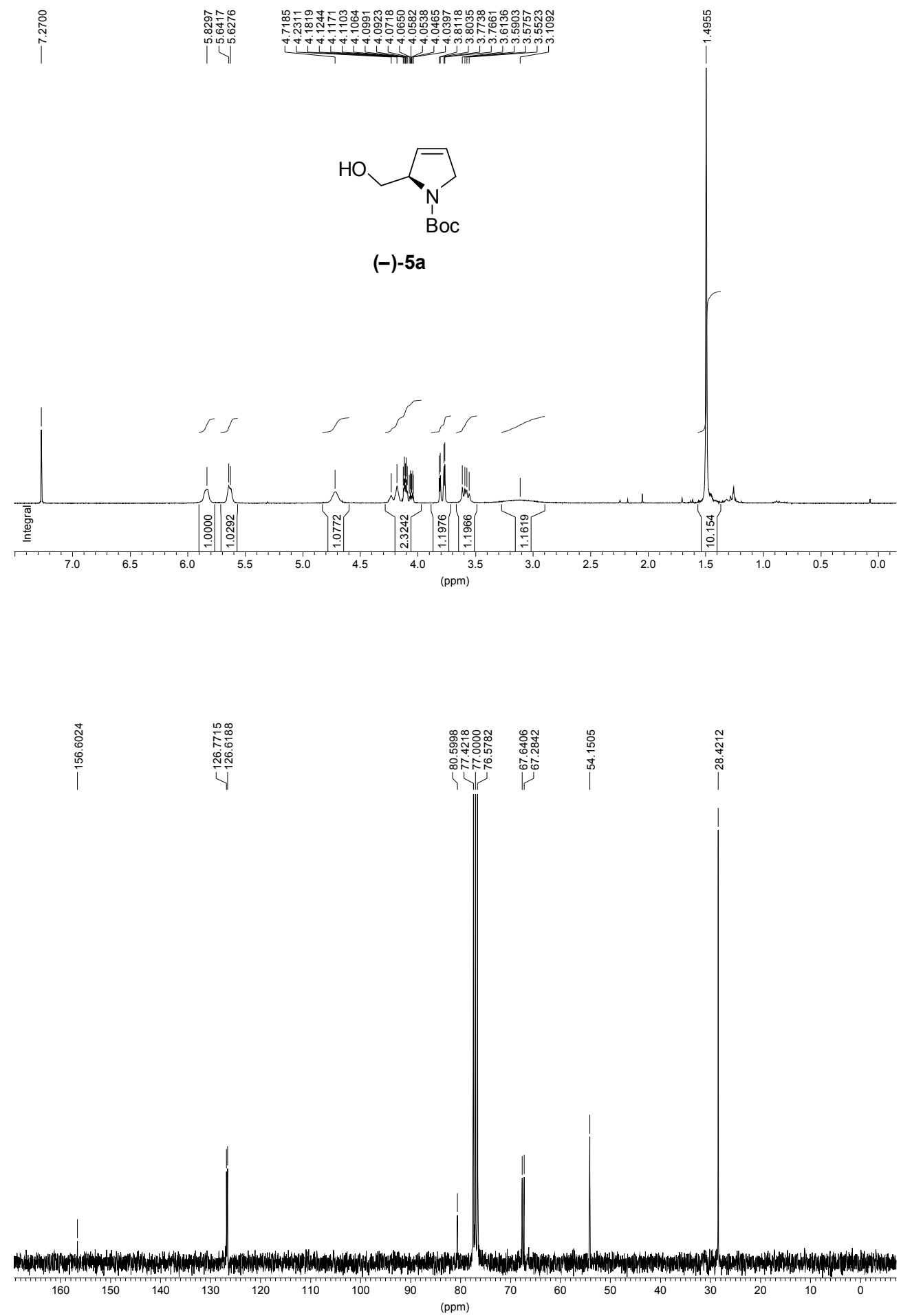

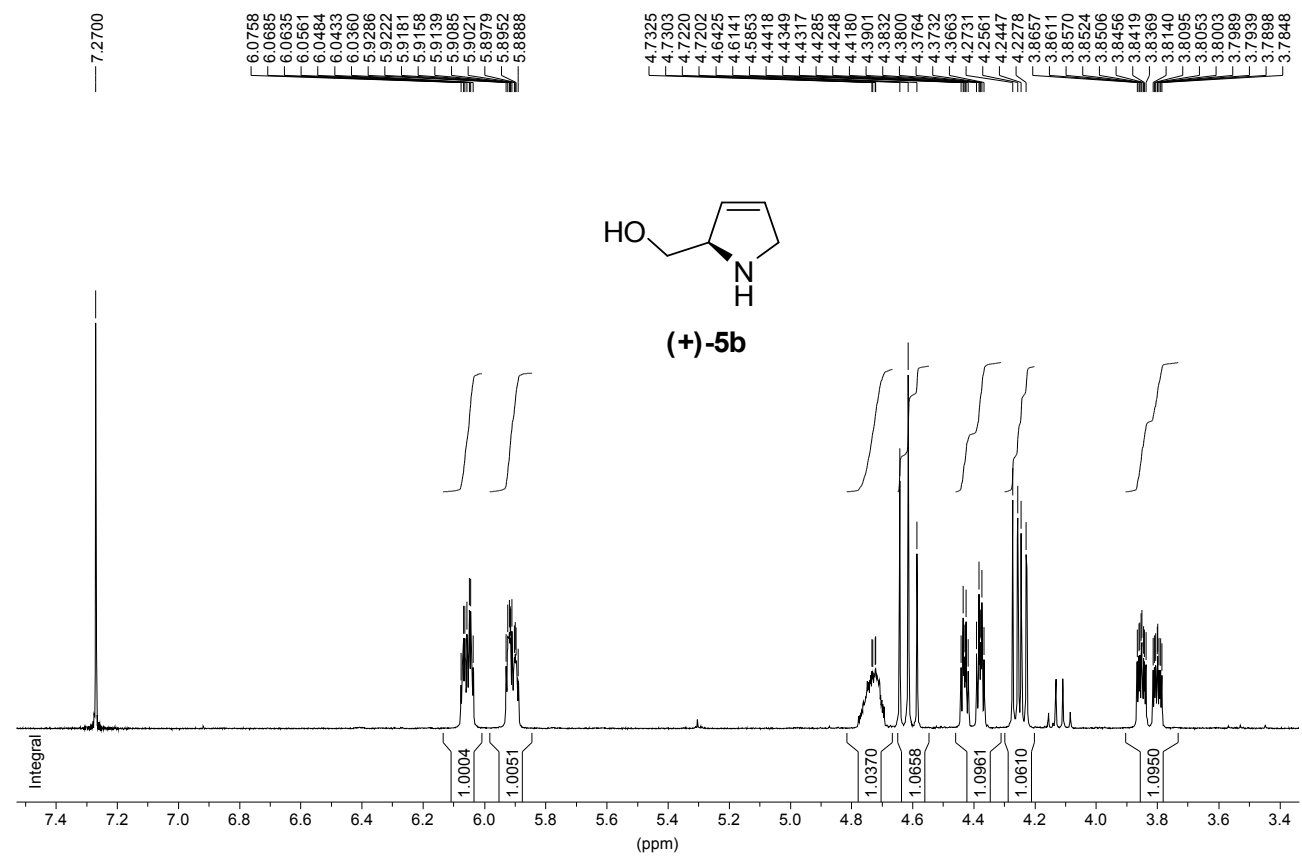

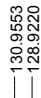

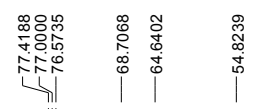

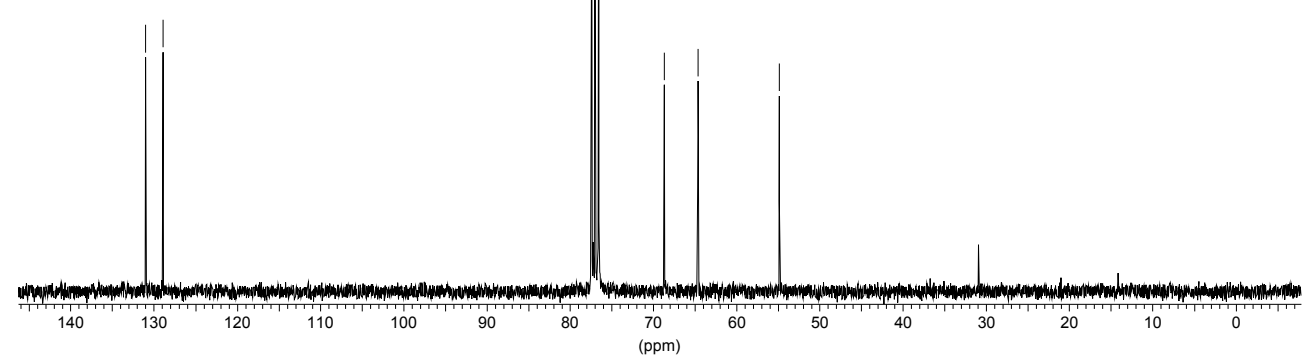




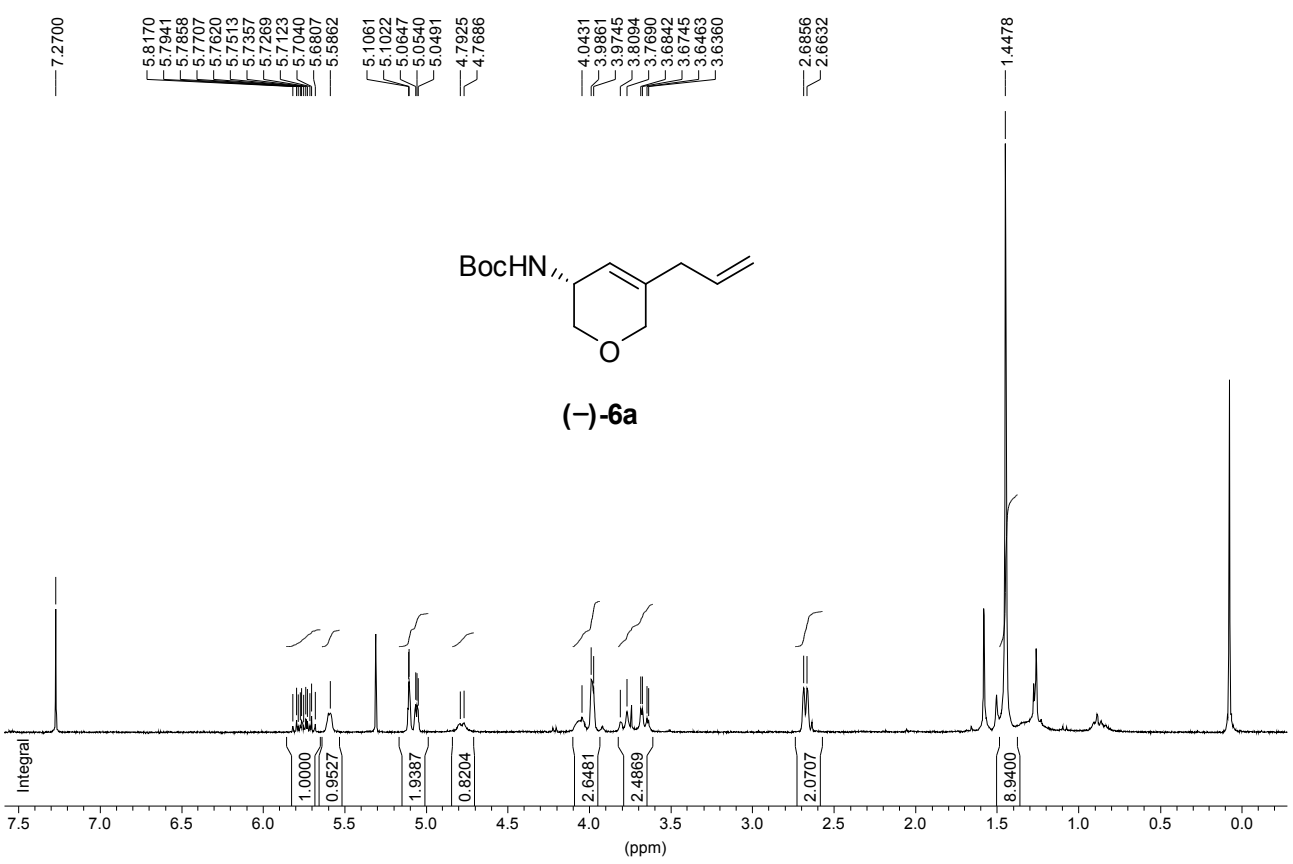

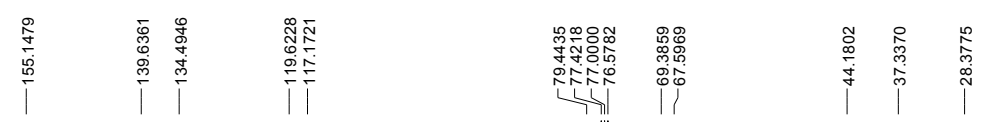

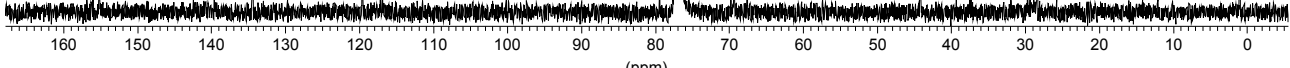




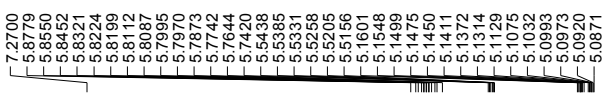

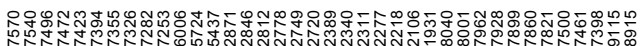
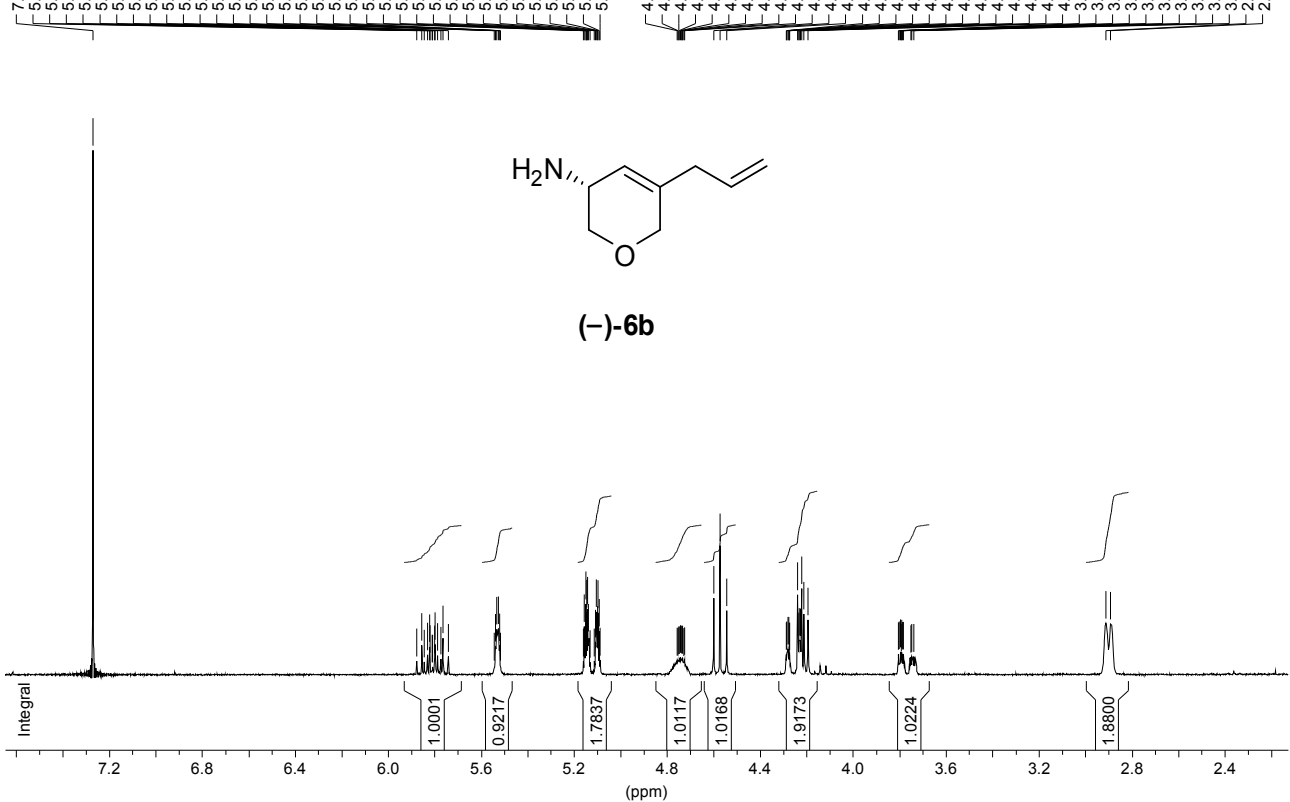

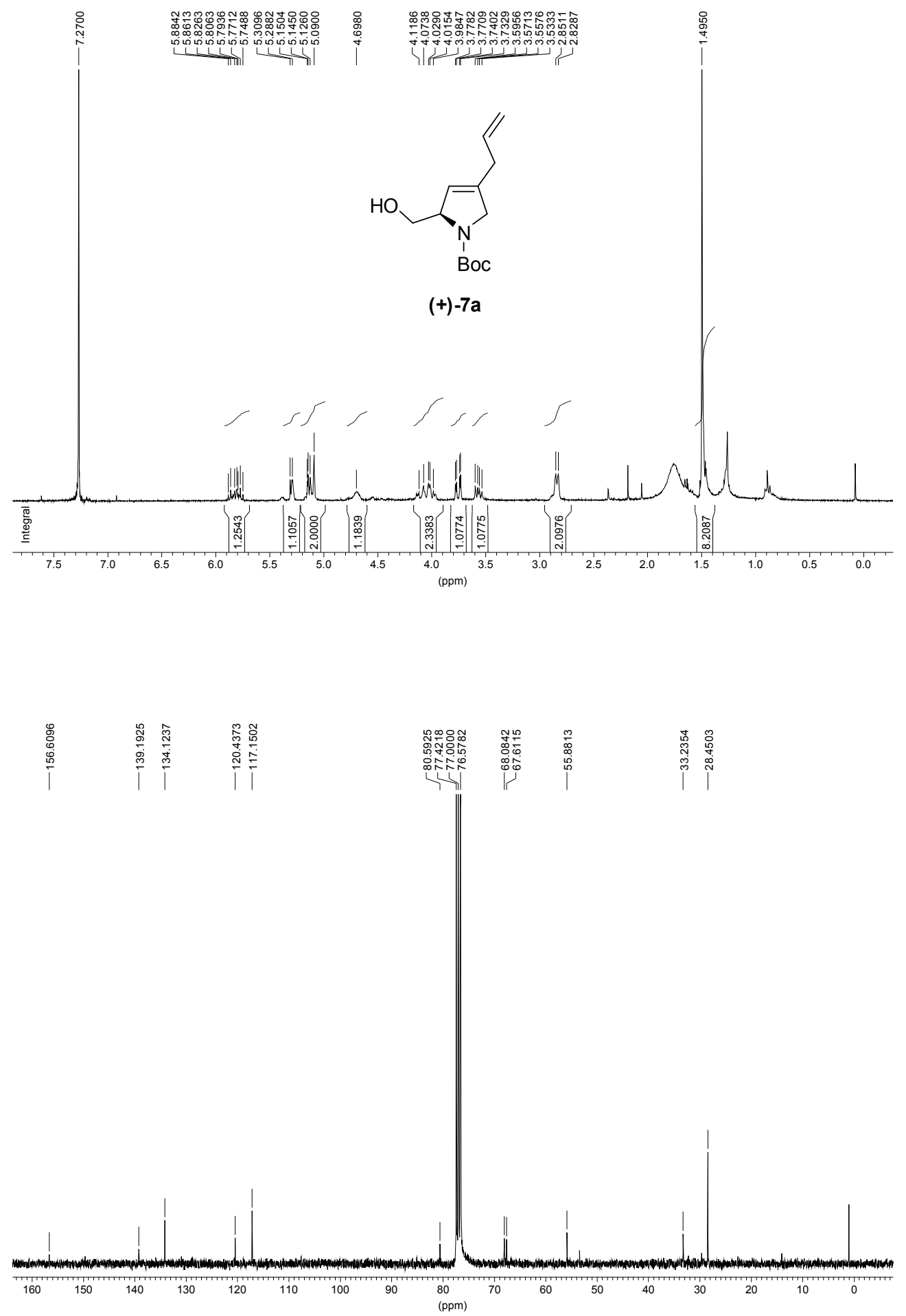


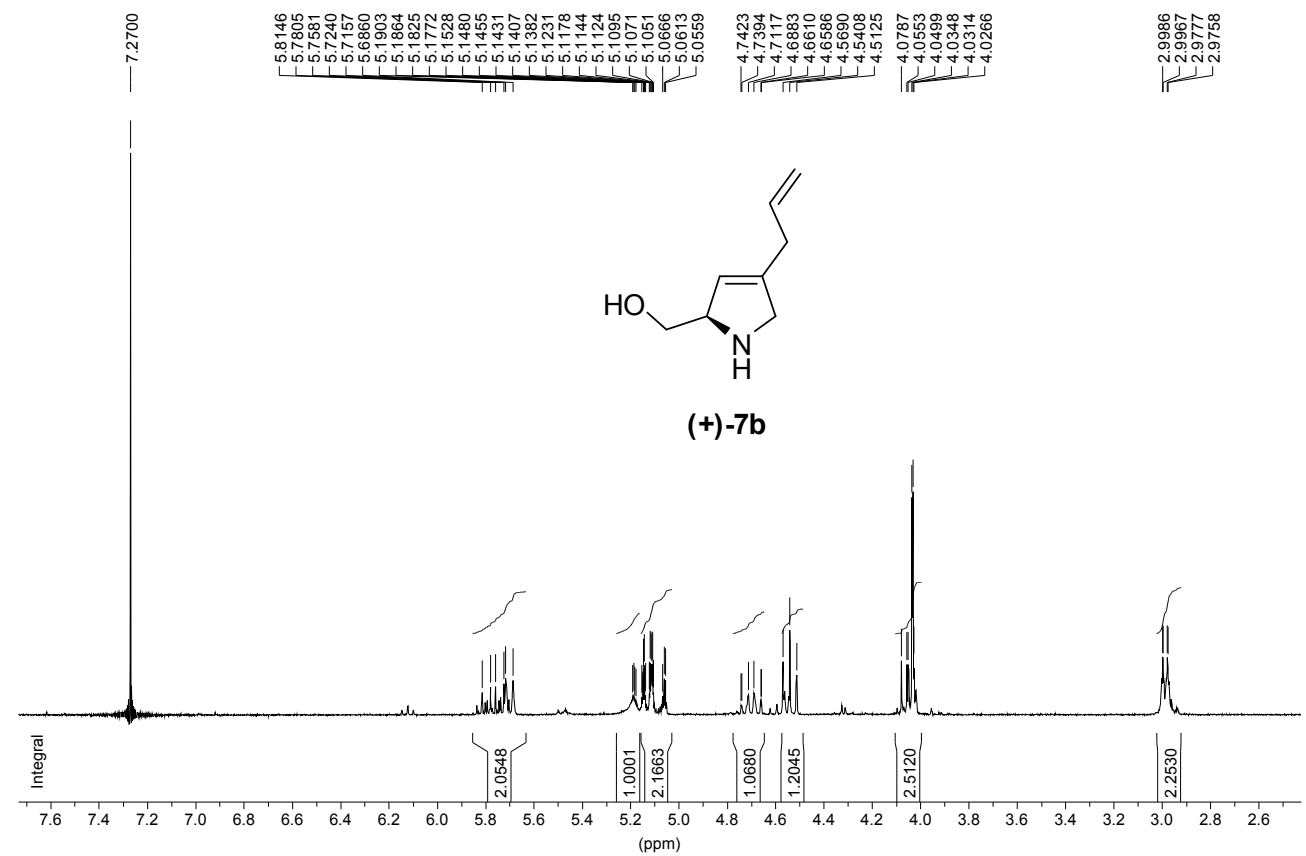

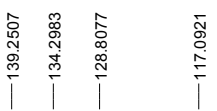

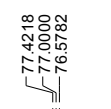

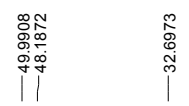

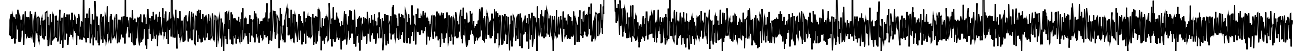

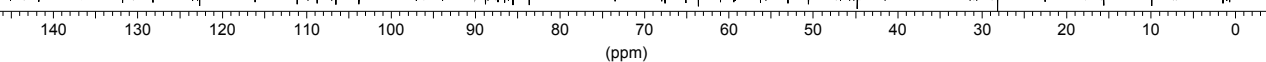




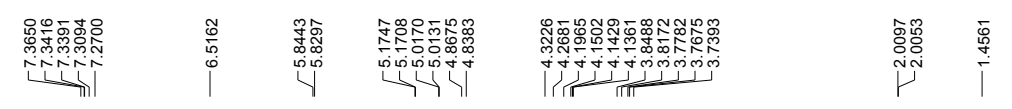

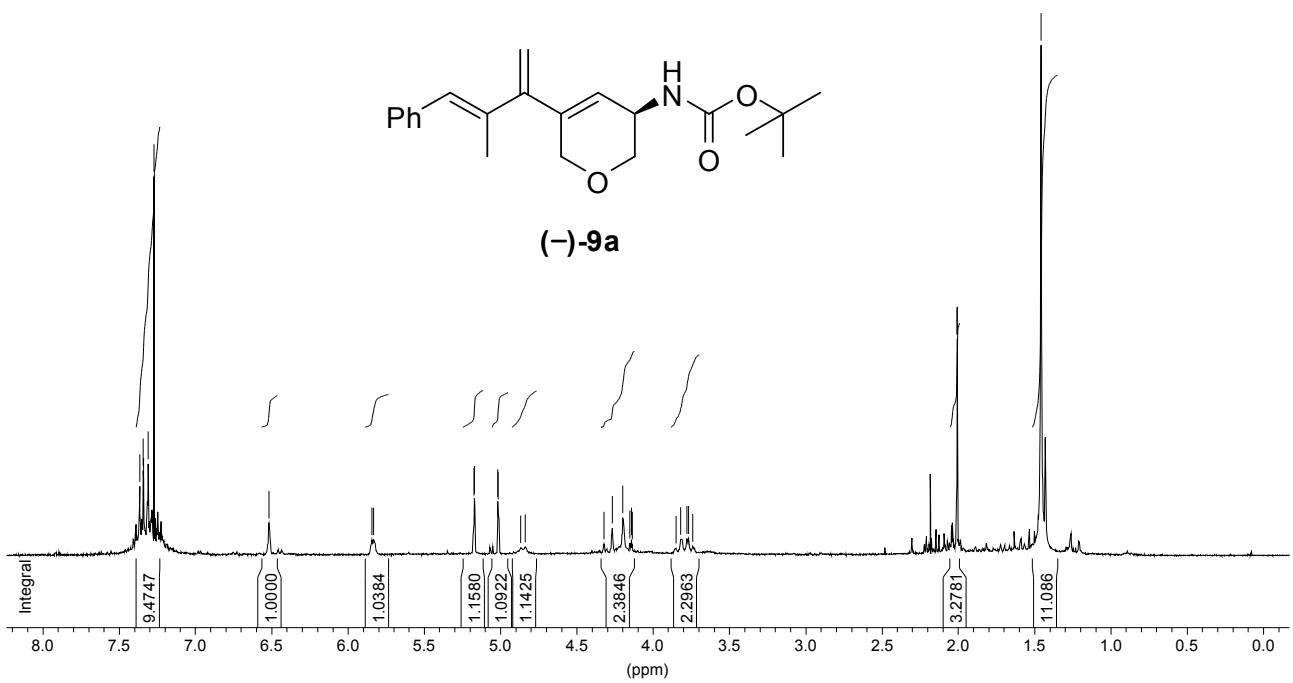

II Hen

\begin{tabular}{|c|}
\hline 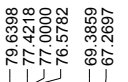 \\
\hline
\end{tabular}

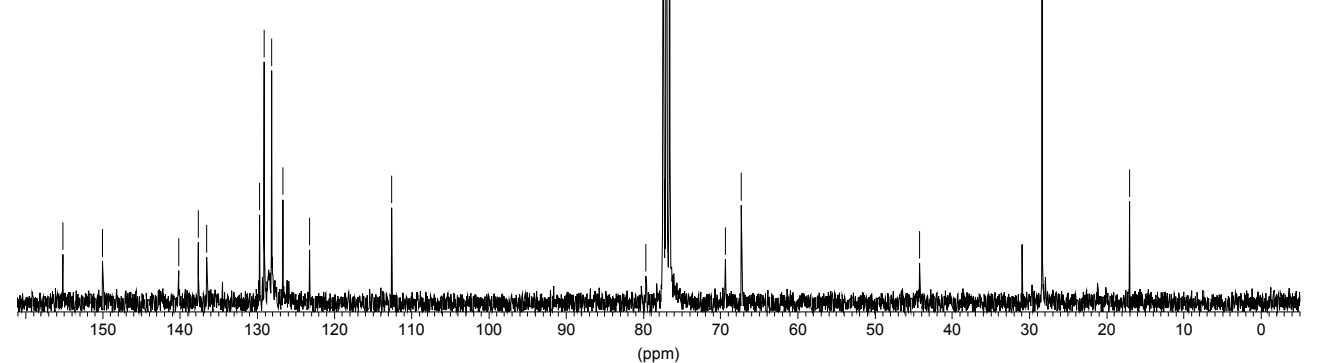




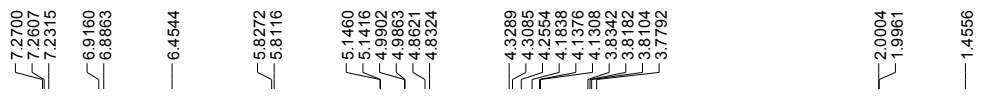<smiles>C=C(/C=C(\C)C1=C[C@H](NC(=O)OC(C)(C)C)COC1)C(=O)Nc1ccccc1</smiles>

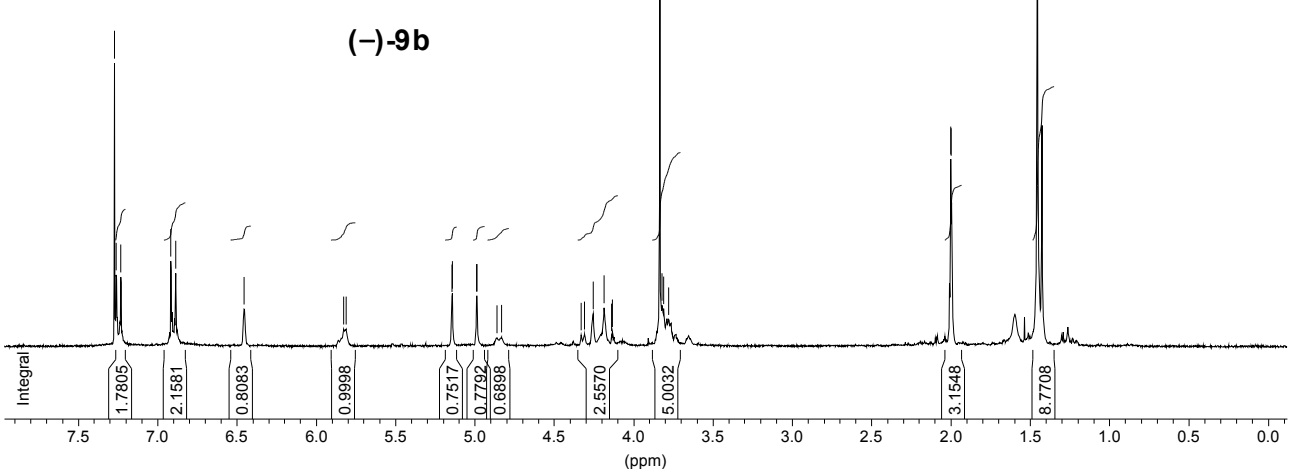

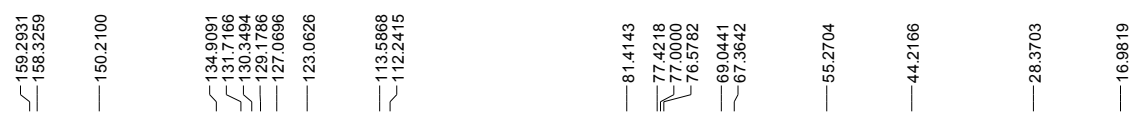

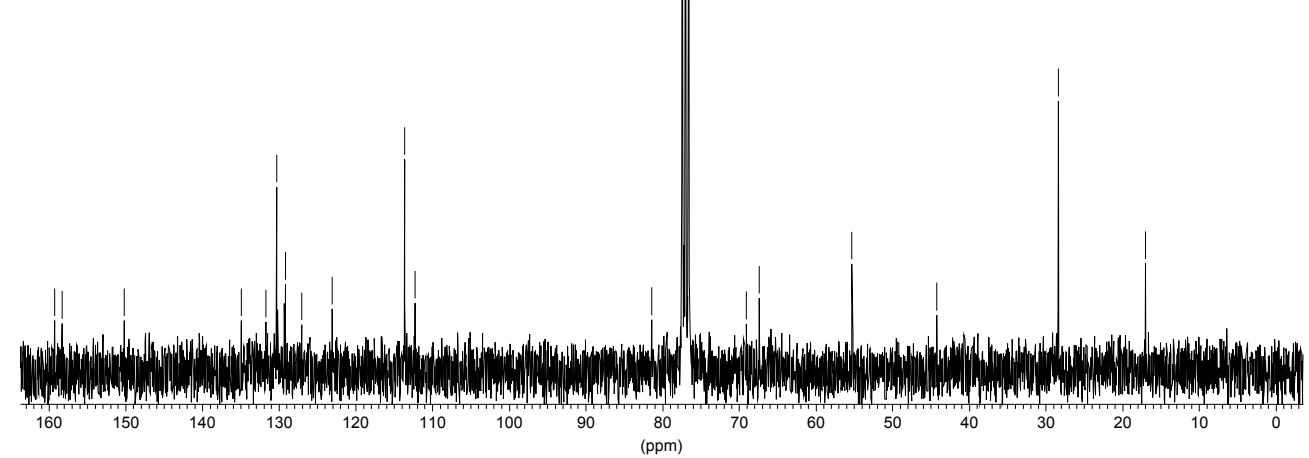




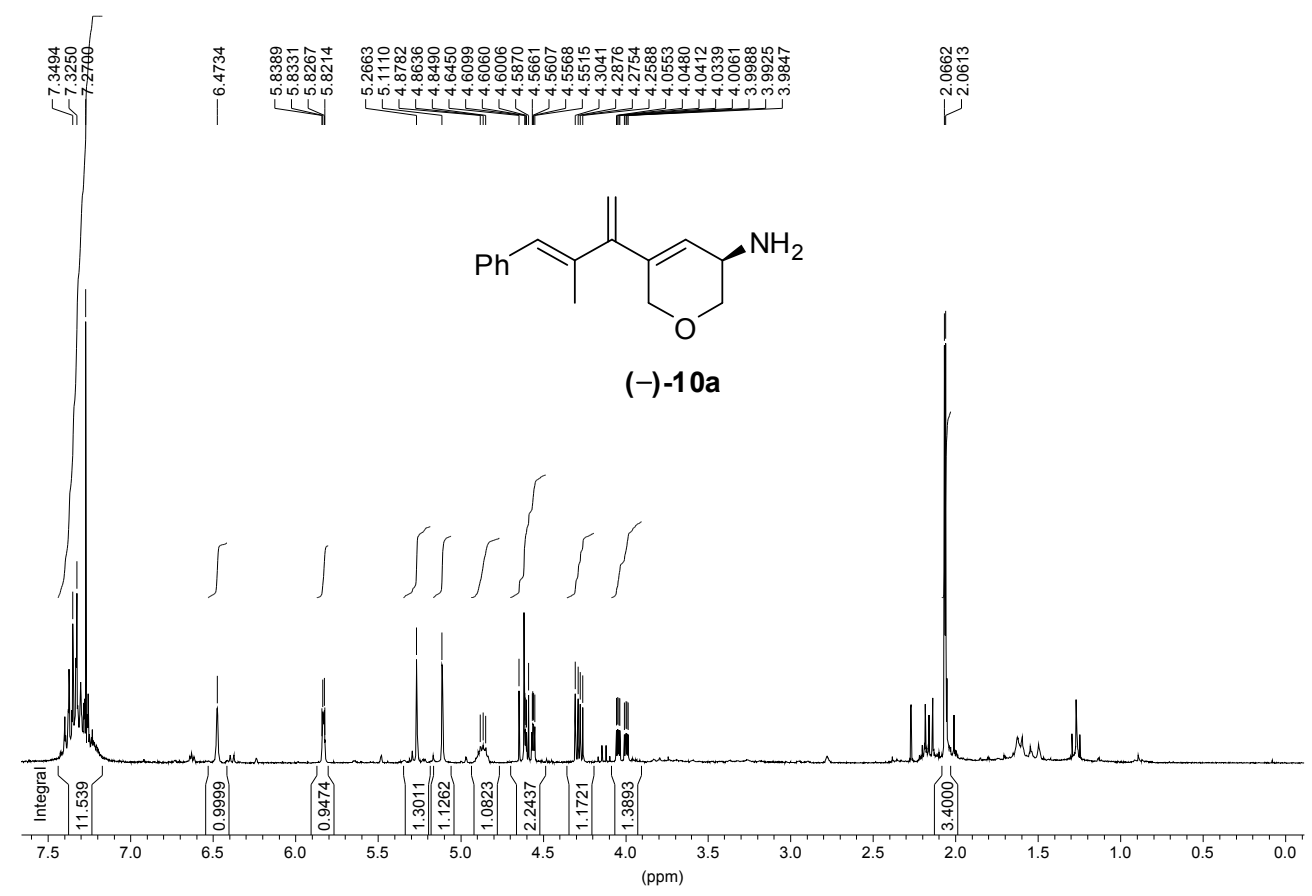




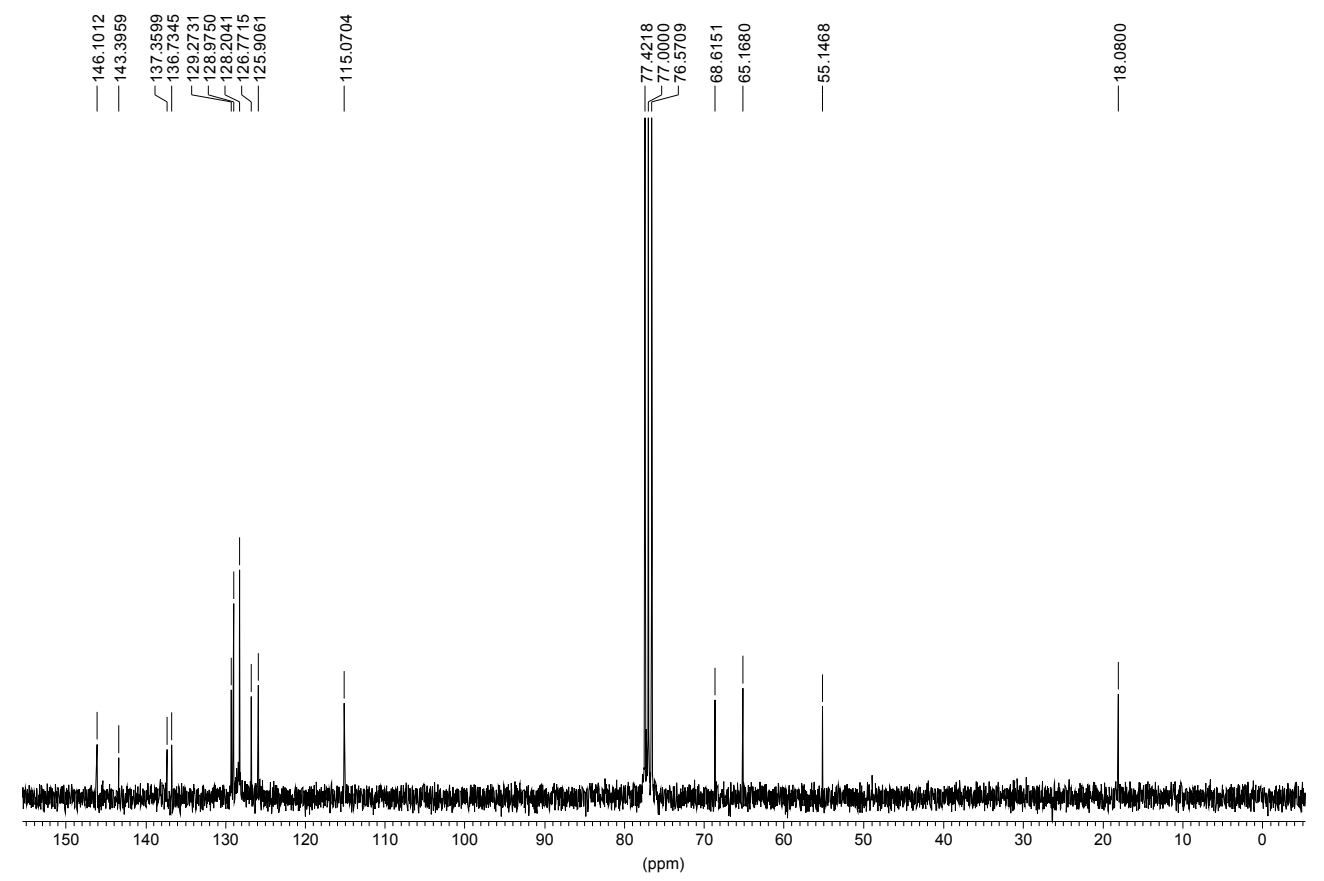




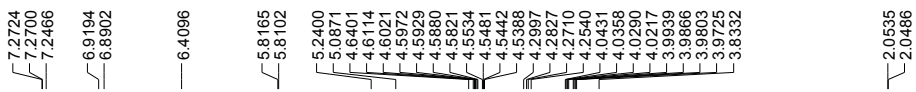

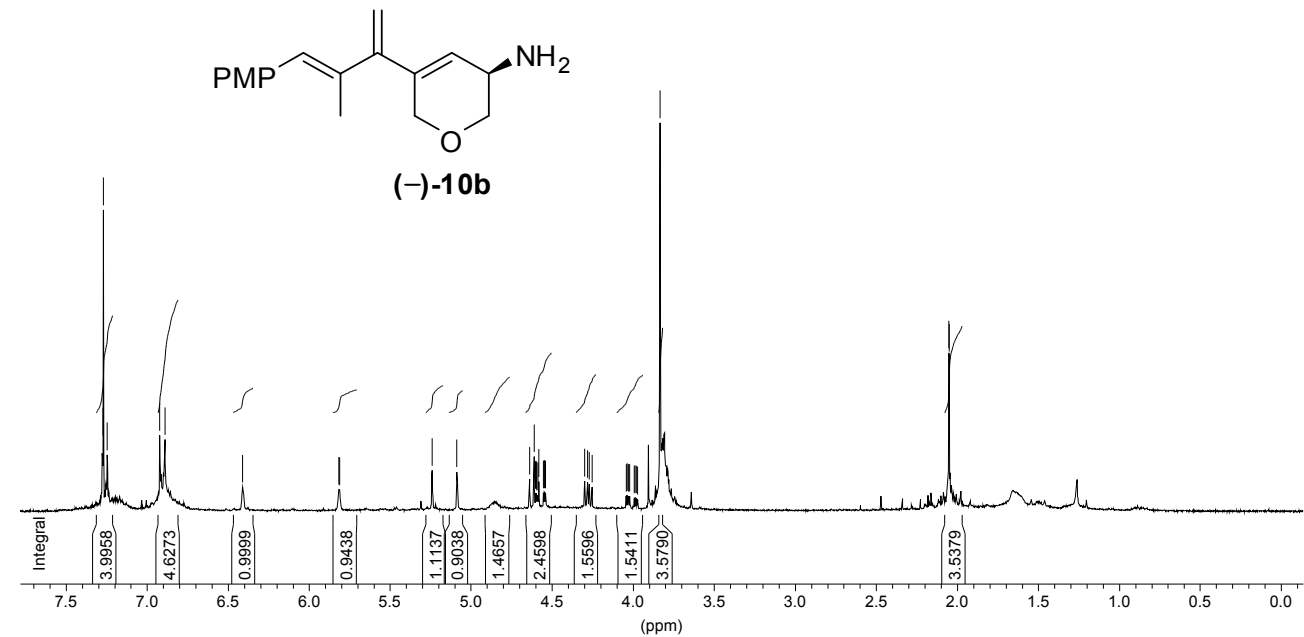

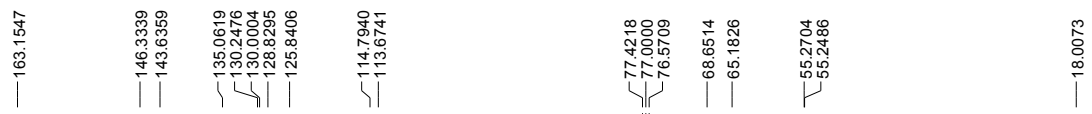

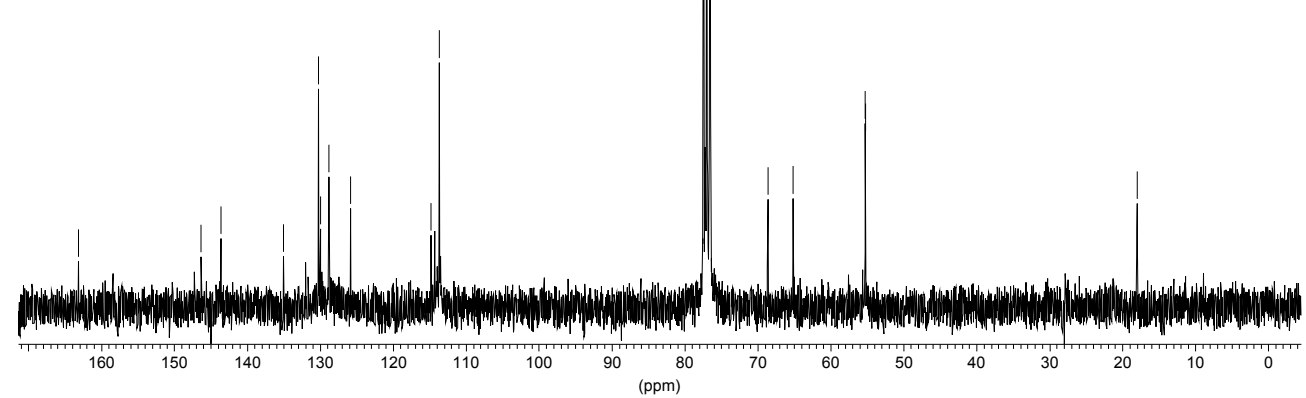



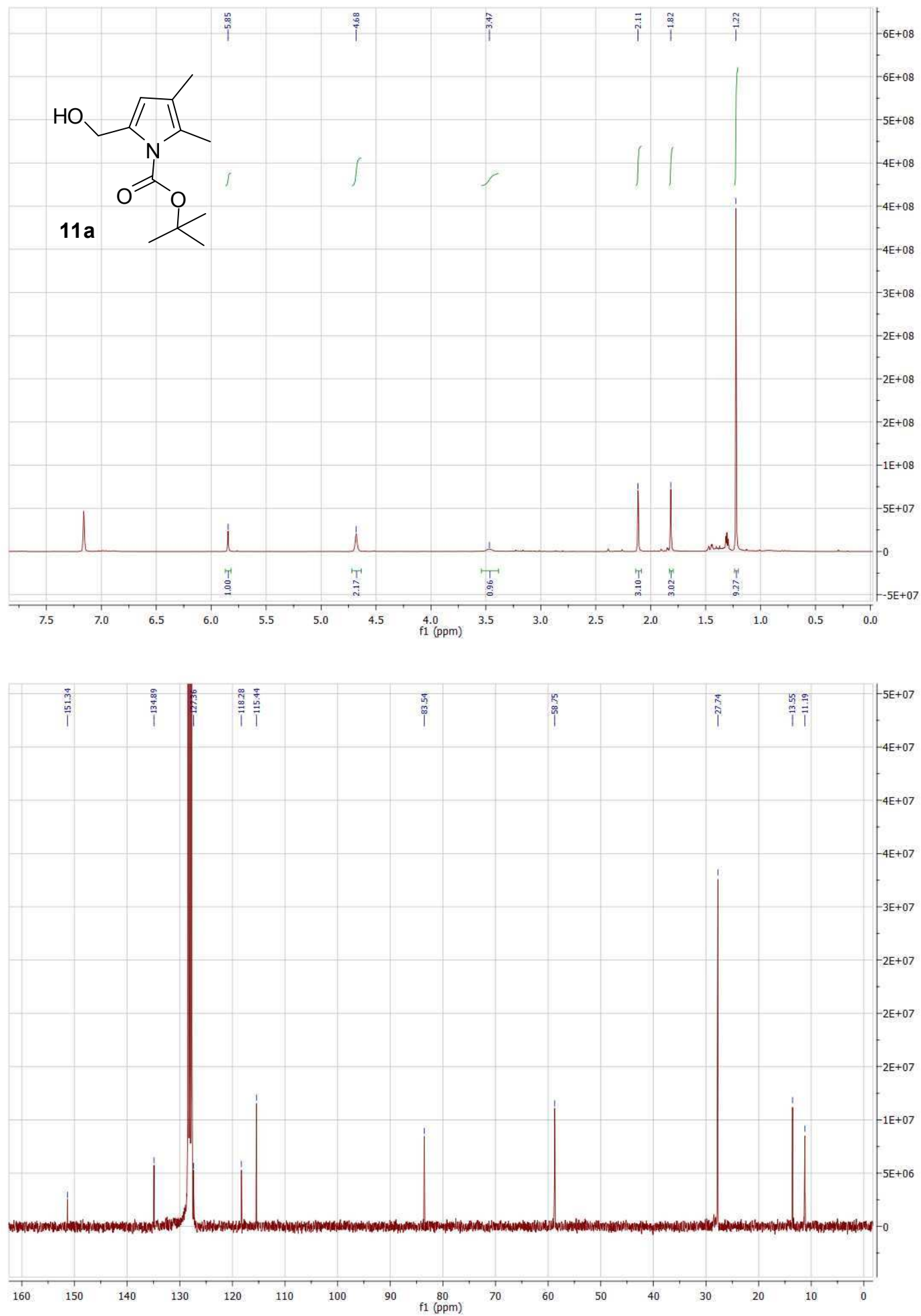

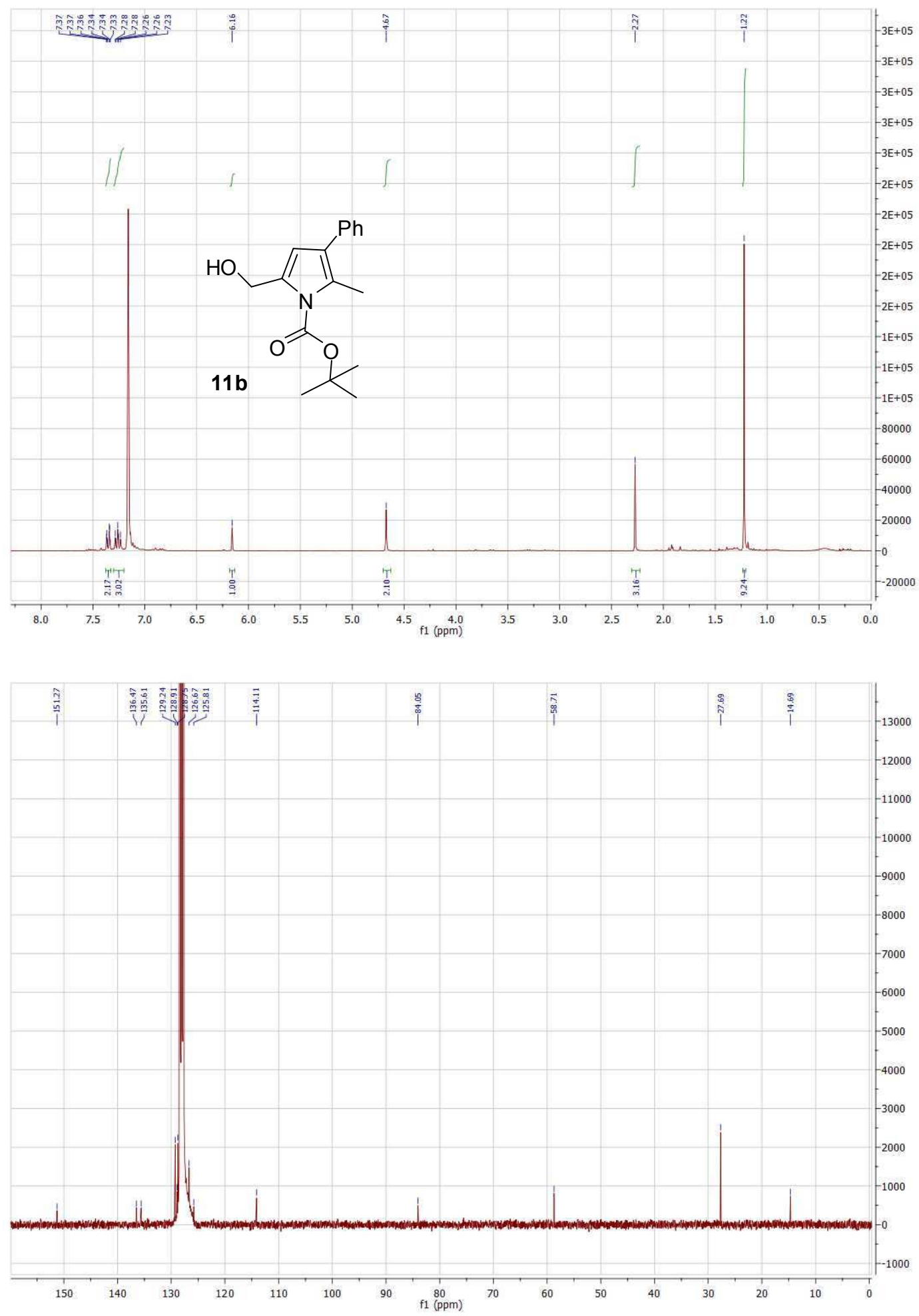